THIS PAGE INTENTIONALLY LEFT BLANK 
Prepared in cooperation with the

Massachusetts Department of Environmental Protection

\section{Refinement and Evaluation of the Massachusetts Firm-Yield Estimator Model Version 2.0}

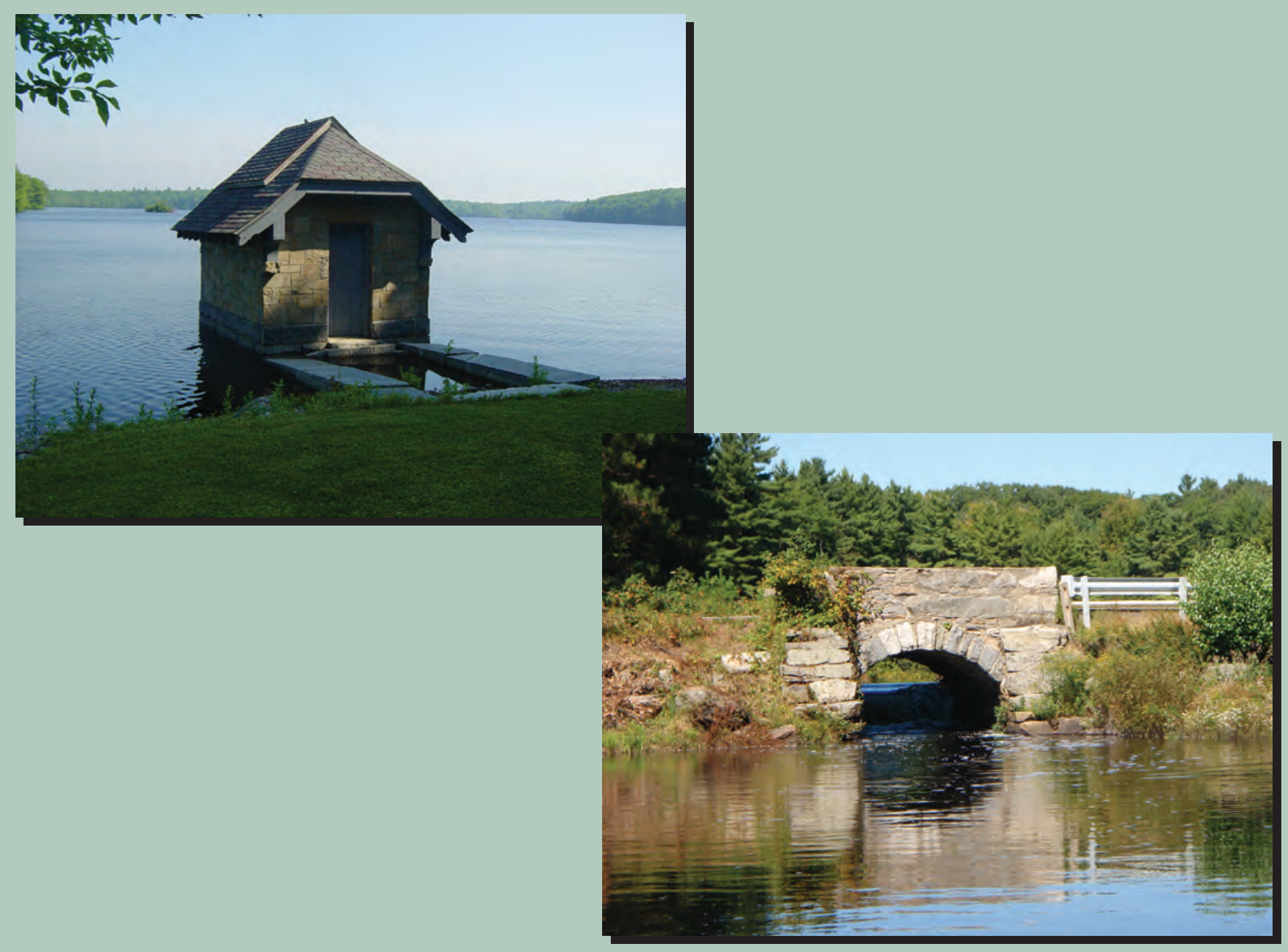

Scientific Investigations Report 2011-5125

U.S. Department of the Interior

U.S. Geological Survey 
Cover. Top: Gatehouse at Flints (Sandy) Pond in Lincoln, Massachusetts. Bottom: Water entering Holden Pond \#1 from Kendall Reservoir, Holden, Massachusetts. 


\section{Refinement and Evaluation of the Massachusetts Firm-Yield Estimator Model Version 2.0}

By Sara B. Levin, Stacey A. Archfield, and Andrew J. Massey

Prepared in cooperation with the

Massachusetts Department of Environmental Protection

Scientific Investigations Report 2011-5125 


\section{U.S. Department of the Interior \\ KEN SALAZAR, Secretary \\ U.S. Geological Survey \\ Marcia K. McNutt, Director}

U.S. Geological Survey, Reston, Virginia: 2011

Revised: October 2011

For more information on the USGS - the Federal source for science about the Earth, its natural and living resources, natural hazards, and the environment, visit http://www.usgs.gov or call 1-888-ASK-USGS.

For an overview of USGS information products, including maps, imagery, and publications, visit http://www.usgs.gov/pubprod

To order this and other USGS information products, visit http://store.usgs.gov

Any use of trade, product, or firm names is for descriptive purposes only and does not imply endorsement by the U.S. Government.

Although this report is in the public domain, permission must be secured from the individual copyright owners to reproduce any copyrighted materials contained within this report.

Suggested citation:

Levin, S.B., Archfield, S.A., and Massey, A.J., 2011, Refinement and evaluation of the Massachusetts firm-yield estimator model version 2.0: U.S. Geological Survey Scientific Investigations Report 2011-5125, 49 p., plus CD-ROM (Also available at http://pubs.usgs.gov/sir/2011/5125.) 


\section{Acknowledgments}

The authors thank Thomas Lamonte and Kari Winfield of the Massachusetts Department of

Environmental Protection and Linda Hutchins of the Massachusetts Department of Conservation and Recreation for their technical reviews of this report.

The authors thank U.S. Geological Survey colleagues Marcus C. Waldron and Robert Dudley for their reviews and Lance Ostiguy for his help in the processing of bathymetric measurement data. 
THIS PAGE INTENTIONALLY LEFT BLANK 


\section{Contents}

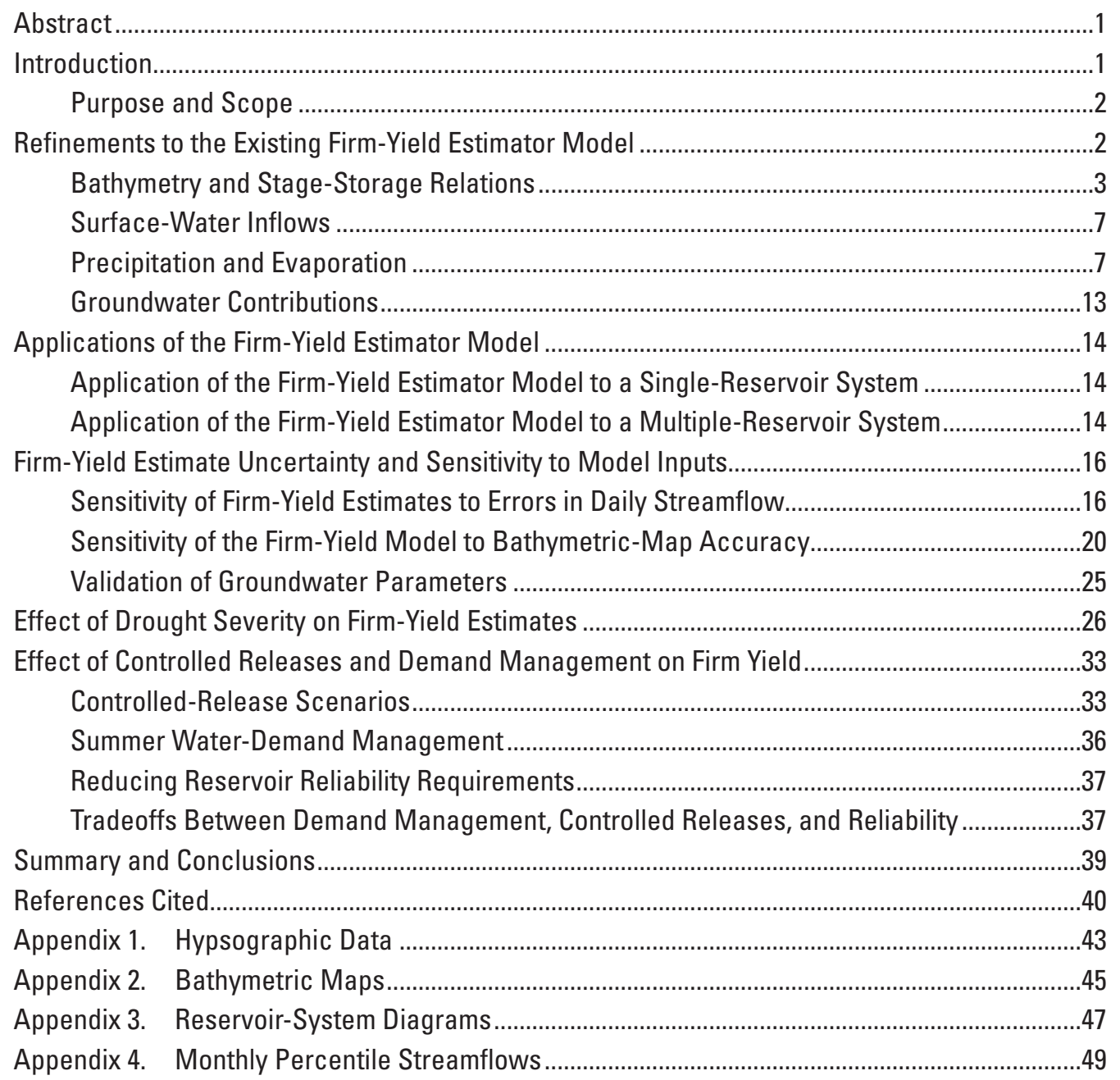




\section{Figures}

1. Map showing locations of drainage areas for 71 drinking-water reservoirs in Massachusetts.

2. Maps showing location of reservoirs and record length at $A$, precipitation stations and validations sites, and $B$, meteorological stations in Massachusetts and vicinity ...12

3. Diagrams showing water balances for a system of reservoirs in which $A$, water is transported by gravity and $B$, water is pumped from Reservoir 1 to Reservoir 2 ..

4. Scatterplots showing $A$, mean percent difference and $B$, standard deviation of the percent difference of daily streamflows generated by the Sustainable Yield Estimator at 18 U.S. Geological Survey gaged sites in Massachusetts.

5. Boxplot showing percent change in firm yield of selected reservoirs in Massachusetts after accounting for potential errors in daily streamflow in $\mathbf{5 0 0}$ Monte Carlo simulations.

6. Diagrams showing original and hypothetical 30-meter transect spacing for Upper Sackett Reservoir in Pittsfield, Massachusetts.

7. Graphs showing $A-C$, percent change in reservoir storage capacity and $D-F$, firm yield, resulting from transect spacings and patterns for three study reservoirs in Massachusetts.

8. Graph showing daily simulated and observed reservoir stage for Atkins Reservoir in Amherst, Massachusetts

9. Graph showing daily simulated and observed reservoir stage for Nagog Pond in Concord, Massachusetts

10. Boxplots showing $A$, percent below average streamflow and $B$, duration of droughts of the 1960s, 1980s, and 2002. C, Percent change in firm yield and $D$, reliability of firm yields when calculated with the droughts of the 1980s or 2002

11. Graph showing maximum monthly releases as a percentile of long-term monthly flows that is possible at various demand ratios for four reservoirs of increasing storage ratio

12. Boxplot showing percent changes of firm yield for Massachusetts reservoirs under various management scenarios

13. Graph showing tradeoff curves depicting the estimated yield in relation to controlled releases under various management scenarios for Upper Leahy Reservoir, in Lee, Massachusetts 


\section{Tables}

1. Massachusetts drinking-water-supply systems and associated reservoirs included in this study.

2. Reservoir basin characteristics and index streamgages in Massachusetts used in the Sustainable Yield Estimator.

3. Nash-Sutcliffe Efficiency of daily precipitation at 33 gaged validation sites in Massachusetts

4. Firm-yield estimates and 2000-2004 water usage for 38 reservoir systems in Massachusetts.

5. Distribution of firm yields resulting from 500 Monte Carlo simulations at selected reservoirs in Massachusetts

6. Reservoir capacity and firm yield for selected reservoirs in Massachusetts calculated using various transect patterns and sampling-point densities.

7. Firm yields and reliability of reservoirs in Massachusetts calculated using three major droughts in the historical record.

8. Water usage and firm yields for reservoir systems in Massachusetts under various controlled-release, demand-management, and reliability scenarios.

\section{Conversion Factors and Datum}

Inch/Pound to SI

\begin{tabular}{lcl}
\hline \multicolumn{1}{c}{ Multiply } & By & \multicolumn{1}{c}{ To obtain } \\
\hline foot $(\mathrm{ft})$ & Length & \\
mile $(\mathrm{mi})$ & 0.3048 & meter $(\mathrm{m})$ \\
& 1.609 & kilometer $(\mathrm{km})$ \\
\hline acre & Area & \\
square mile $\left(\mathrm{mi}^{2}\right)$ & 4,047 & square meter $\left(\mathrm{m}^{2}\right)$ \\
\hline & 2.590 & square kilometer $\left(\mathrm{km}^{2}\right)$ \\
\hline million gallons $(\mathrm{Mgal})$ & Volume & \\
cubic foot $\left(\mathrm{ft}^{3}\right)$ & 3,785 & cubic meter $\left(\mathrm{m}^{3}\right)$ \\
\hline & 0.02832 & cubic meter $\left(\mathrm{m}^{3}\right)$ \\
\hline foot per day $(\mathrm{ft} / \mathrm{d})$ & Flow rate & \\
cubic foot per second $\left(\mathrm{ft}^{3} / \mathrm{s}\right)$ & 0.3048 & meter per day $(\mathrm{m} / \mathrm{d})$ \\
million gallons per day $(\mathrm{Mgal} / \mathrm{d})$ & 0.02832 & cubic meter per second $\left(\mathrm{m}^{3} / \mathrm{s}\right)$ \\
\hline & 0.04381 & cubic meter per second $\left(\mathrm{m}^{3} / \mathrm{s}\right)$ \\
\hline foot squared per day $\left(\mathrm{ft}^{2} / \mathrm{d}\right)$ & Transmissivity* & \\
\hline
\end{tabular}

Vertical coordinate information is referenced to the North American Vertical Datum of 1988 (NAVD 88).

Horizontal coordinate information is referenced to the North American Datum of 1983 (NAD 83).

Transmissivity: The standard unit for transmissivity is cubic foot per day per square foot times foot of aquifer thickness $\left[\left(\mathrm{ft}^{3} / \mathrm{d}\right) / \mathrm{ft}^{2}\right] \mathrm{ft}$. In this report, the mathematically reduced form, foot squared per day $\left(\mathrm{ft}^{2} / \mathrm{d}\right)$, is used for convenience. 
THIS PAGE INTENTIONALLY LEFT BLANK 


\title{
Refinement and Evaluation of the Massachusetts Firm-Yield Estimator Model Version 2.0
}

\author{
By Sara B. Levin, Stacey A. Archfield, and Andrew J. Massey
}

\section{Abstract}

The firm yield is the maximum average daily withdrawal that can be extracted from a reservoir without risk of failure during an extended drought period. Previously developed procedures for determining the firm yield of a reservoir were refined and applied to 38 reservoir systems in Massachusetts, including 25 single- and multiple-reservoir systems that were examined during previous studies and 13 additional reservoir systems. Changes to the firm-yield model include refinements to the simulation methods and input data, as well as the addition of several scenario-testing capabilities. The simulation procedure was adapted to run at a daily time step over a 44-year simulation period, and daily streamflow and meteorological data were compiled for all the reservoirs for input to the model.

Another change to the model-simulation methods is the adjustment of the scaling factor used in estimating groundwater contributions to the reservoir. The scaling factor is used to convert the daily groundwater-flow rate into a volume by multiplying the rate by the length of reservoir shoreline that is hydrologically connected to the aquifer. Previous firm-yield analyses used a constant scaling factor that was estimated from the reservoir surface area at full pool. The use of a constant scaling factor caused groundwater flows during periods when the reservoir stage was very low to be overestimated. The constant groundwater scaling factor used in previous analyses was replaced with a variable scaling factor that is based on daily reservoir stage. This change reduced instability in the groundwater-flow algorithms and produced more realistic groundwater-flow contributions during periods of low storage.

Uncertainty in the firm-yield model arises from many sources, including errors in input data. The sensitivity of the model to uncertainty in streamflow input data and uncertainty in the stage-storage relation was examined. A series of Monte Carlo simulations were performed on 22 reservoirs to assess the sensitivity of firm-yield estimates to errors in dailystreamflow input data. Results of the Monte Carlo simulations indicate that underestimation in the lowest stream inflows can cause firm yields to be underestimated by an average of 1 to 10 percent. Errors in the stage-storage relation can arise when the point density of bathymetric survey measurements is too low. Existing bathymetric surfaces were resampled using hypothetical transects of varying patterns and point densities in order to quantify the uncertainty in stage-storage relations. Reservoir-volume calculations and resulting firm yields were accurate to within 5 percent when point densities were greater than 20 points per acre of reservoir surface.

Methods for incorporating summer water-demandreduction scenarios into the firm-yield model were developed as well as the ability to relax the no-fail reliability criterion. Although the original firm-yield model allowed monthly reservoir releases to be specified, there have been no previous studies examining the feasibility of controlled releases for downstream flows from Massachusetts reservoirs. Two controlled-release scenarios were tested - with and without a summer water-demand-reduction scenario-for a scenario with a no-fail criterion and a scenario that allows for a 1-percent failure rate over the entire simulation period. Based on these scenarios, about one-third of the reservoir systems were able to support the flow-release scenarios at their 2000-2004 usage rates. Reservoirs with higher storage ratios (reservoir storage capacity to mean annual streamflow) and lower demand ratios (mean annual water demand to annual firm yield) were capable of higher downstream release rates. For the purposes of this research, all reservoir systems were assumed to have structures which enable controlled releases, although this assumption may not be true for many of the reservoirs studied.

\section{Introduction}

The determination of available water for, and management of, public water supplies in Massachusetts drinking-water reservoirs has become increasingly complex as regulators try to provide adequate streamflows for ecological communities as well as increasing demands for drinking water. Controlled releases from reservoirs to satisfy ecological demands may impose further limits on the yield from a reservoir. A reservoir failure occurs when a reservoir is unable to provide sufficient water to meet demand. Reservoirs with high water demand relative to their firm yield are particularly 
at risk of failing during periods of drought. In order to ensure that reservoirs have sufficient water available even during extreme droughts, the Massachusetts Department of Environmental Protection (MassDEP) requires public water suppliers to estimate the firm yield of their reservoir systems.

The firm yield of a reservoir is defined as the maximum yield that can be delivered from a system without failure, even during a severe drought. To facilitate the calculation of a system's firm yield, MassDEP developed the FirmYield Estimator (FYE) guidance document (Massachusetts Department of Environmental Protection, 1996) and model (Massachusetts Department of Environmental Protection, 2000). The FYE model uses the water-balance equation to estimate reservoir storage over a period of time that includes the most extreme drought of record. The firm yield of a reservoir is calculated by solving the water-balance equation for each month of the simulation period with an initial yield of 0 million gallons per day $(\mathrm{Mgal} / \mathrm{d})$. If the reservoir storage is does not fully deplete during the simulation, yield is increased, and the water-balance equation is solved again. Iteration continues with increasing yield until a reservoir failure occurs. The firm yield is the maximum yield that can be used in the water-balance equation without causing the reservoir to fail during the simulation period. Waldron and Archfield (2006) calculated firm yields for 25 single- and multiple-reservoir systems in Massachusetts and examined the sensitivity of the FYE model to various model inputs. Archfield and Carlson (2006) further refined the FYE model by developing a procedure to estimate groundwater flows into and out of reservoir storage.

Growing demand for public water supply in Massachusetts may compete with ecosystem water needs, and there is increasing interest in ensuring that streams have adequate water to support aquatic communities. Reservoir impoundments can alter the natural streamflow patterns in reaches downstream of the dam. During periods when reservoir stage is lower than the spillway elevation, water cannot spill over the dam into the downstream reaches and can alter the characteristic pattern of high and low flows for that stream reach. These alterations to streamflow can be detrimental to downstream ecosystems and aquatic communities. Controlled releases from reservoir storage can be implemented to alleviate downstream ecosystem stress; however, these releases decrease reservoir storage. In some cases, meeting environmental streamflow needs during a severe drought may decrease reservoir yield enough that human needs cannot be met. Although the FYE model allows for user-specified monthly controlled releases, there have been no statewide applications of the model to examine the feasibility of implementing controlled releases in Massachusetts drinking-water reservoirs.

Water suppliers struggling to meet drinking-water demands or wishing to offset the effects of controlled flow releases may consider demand-management strategies such as outdoor summer water-use restrictions, or they may consider relaxing the no-fail reliability criterion of the reservoir, or a combination of these two strategies. Each strategy has advantages and disadvantages, and the appropriate solution may differ from one reservoir to another. Summer water-use restrictions limit non-essential water use during drought periods but may not conserve enough water to sustain use through long, multiyear droughts when winter and spring flows cannot replenish the reservoir. Relaxing the reliability requirement of a reservoir may provide a boost in yield, but reservoirs can run the risk of failure during very severe droughts. The MassDEP guidance document and original FYE model do not allow for user-specified reliability criteria or demand-management scenarios. For the current study, modifications to the FYE were developed to allow these options in order to assess the relative benefits of these operational strategies.

\section{Purpose and Scope}

The purpose of this report is to document the methods used to estimate firm yield for Massachusetts reservoirs and to examine the factors that affect the firm-yield estimates. The report describes several refinements to the existing firm-yield model, including simulation at a daily time step; refinement and validation of groundwater parameters; improved input data for streamflow, precipitation, and evaporation; and modification of the model to allow for demand-management scenarios and relaxed reliability criteria. Firm yields were calculated using the updated data and methodology for 25 reservoir systems previously studied as well as 13 reservoir systems that were not examined in previous firm-yield studies. The sensitivity of the model to bathymetric-map accuracy, drought severity, and uncertainty in input data was examined. Finally, the tradeoffs between controlled releases and demandmanagement scenarios were examined.

\section{Refinements to the Existing Firm-Yield Estimator Model}

The Massachusetts Department of Environmental Protection (MassDEP) guidance document details a procedure for calculating the firm yield of a reservoir in Massachusetts. To facilitate this calculation, the Firm-Yield Estimator (FYE) model (Massachusetts Department of Environmental Protection, 1996) was developed on the basis of this methodology. The FYE model defines the firm yield as the maximum yield at which a water-supply reservoir or system of reservoirs can operate without failure during the drought of record. Waldron and Archfield (2006) examined the sensitivity of the FYE model to various inputs required by the model and evaluated the model's overall procedure for estimating the firm yield for surface-water-dominated reservoirs in Massachusetts. 
Archfield and Carlson (2006) expanded the methodology to include a set of equations used to estimate groundwater contributions to the reservoir water balance.

Because of the difficulty in obtaining daily values for all of the water-balance components, previous firm-yield analyses in Massachusetts have been performed using a monthly time step. Fennessey (1995) showed that firm yields estimated at a monthly time step are higher than yields estimated from data at a daily time step. The MassDEP guidance document specifies a regression-based correction factor that is used to adjust the firm yield for errors introduced by using a monthly time step instead of a daily time step. However, the applicability and validation of the correction factor remains unverified for Massachusetts reservoirs. New advances in daily streamflow estimation, however, have made it feasible to run the FYE model at a daily time step. Running the model at a daily time step eliminates the need for a correction factor.

With a daily time step and units of volume, the reservoir water-balance equation is:

$$
S_{i}=A r_{i}\left(P_{i}-E_{i}\right)+A w_{i} Q_{s t_{i}}-\alpha_{i} Q_{y}-Q_{r_{i}}-Q_{s p_{i}}-Q_{o w_{i}} \pm Q_{g w_{i}}+S_{i-1}
$$

where

$$
\begin{aligned}
i \quad= & \text { daily simulation time step; } \\
S_{i} \quad= & \text { volume of water in usable storage for the } \\
& \text { current day, in million gallons; } \\
A w_{i} \quad= & \text { reservoir drainage area, in square miles; } \\
Q_{s t i}= & \text { streamflow per unit drainage area, in miles } \\
A r_{i} \quad= & \text { area of the reservoir surface, in square } \\
& \text { miles }^{1} ; \\
P_{i} \quad= & \text { precipitation, in miles }^{1} ; \\
E_{i} \quad= & \text { evaporation from the reservoir surface, in } \\
& \text { miles }^{1} ; \\
Q_{g w i} \quad= & \text { groundwater contributions or losses for the } \\
& \quad \text { current day, in million gallons; } \\
\alpha_{i} \quad= & \text { peak-usage factor, dimensionless; } \\
Q_{y}= & \text { yield for the current day, in million gallons; } \\
Q_{r r i} \quad= & \text { controlled release, in million gallons; } \\
Q_{s p i}= & \text { uncontrolled spill, in million gallons; and } \\
Q_{o w i}= & \text { withdrawal from the reservoir by other } \\
& \text { users, in million gallons. }
\end{aligned}
$$

The firm yield is calculated by successively solving the water-balance equation over the period of record, starting at a full pool volume and increasing the yield at each repetition. The firm yield is the maximum yield volume that can be used without causing the reservoir volume to decrease to zero. For this study, firm yields were determined for 71 reservoirs -45 previously studied reservoirs and 26 new reservoirs - belonging to 38 reservoir systems (fig. 1; table 1). For the purposes of this study, a reservoir system refers to one or more reservoirs

\footnotetext{
${ }^{1}$ Precipitation, streamflow, and evaporation are in units of length and, when multiplied by $A_{r i}$, become volumes (in cubic miles) that are converted to million gallons.
}

that are hydrologically connected. Firm yields for previously studied reservoir systems were re-estimated using the revised FYE-model methods and data. These revised firm yields supersede estimates of firm yield from Waldron and Archfield (2006) and Archfield and Carlson (2006). Previous estimates of firm yields are no longer considered valid.

Reservoir-specific characteristics that affect model estimates of firm yield include the stage-storage relation, storage capacity, streamflows, direct precipitation, and evaporation from the reservoir surface. For reservoirs that are connected to an aquifer, the aquifer transmissivity and surficial geology underlying the reservoir also affect groundwater flows to and from the reservoir.

\section{Bathymetry and Stage-Storage Relations}

Bathymetric surveys were completed for 26 reservoirs that were not previously studied. Surveys were completed during the spring months of 2008 and 2009 when reservoirs were full or nearly full. Reservoir depths were determined with a narrow-beam echo sounder along transects across each reservoir. The location of each depth measurement was determined with a global positioning system (GPS). Reservoirspillway elevations and intake elevations were provided by the public water supplier. In order to convert water depths to bottom elevations, the water depths were first adjusted to account for the reservoir stage on the day of the survey by adding the distance between the water surface and the spillway to each measurement. The reservoir bottom elevation was then calculated as the difference between the spillway elevation and the corrected depth measurements.

The GPS data were differentially corrected and imported into a geographic information system (GIS). Reservoir outlines were digitized from 1:5,000 digital orthoimagery (Office of Geographic and Environmental Information, 2005). Smoothed reservoir contours were generated from bottom elevations using a spatially interpolated grid in ArcINFO. A triangulated irregular network (TIN) model was generated using GPS position, bottom elevation, and the digitized reservoir shoreline. Reservoir volumes and surface areas were computed using the TIN surface model at 2-foot (ft) intervals. This method calculates the volume at each grid cell of the bottom-elevation map, making it more accurate than estimating volume from surface contours alone. Stage-storage relations developed from the bathymetric data were used to estimate reservoir surface area and reservoir stage based on reservoir-storage volumes at each daily time step during the simulation. Interpolation was used to estimate these quantities at volumes that fall between contour intervals. Stage-storage relations and bottom-elevation maps for reservoirs that were not previously studied by Waldron and Archfield (2006) are included in appendixes 1 and 2 . 

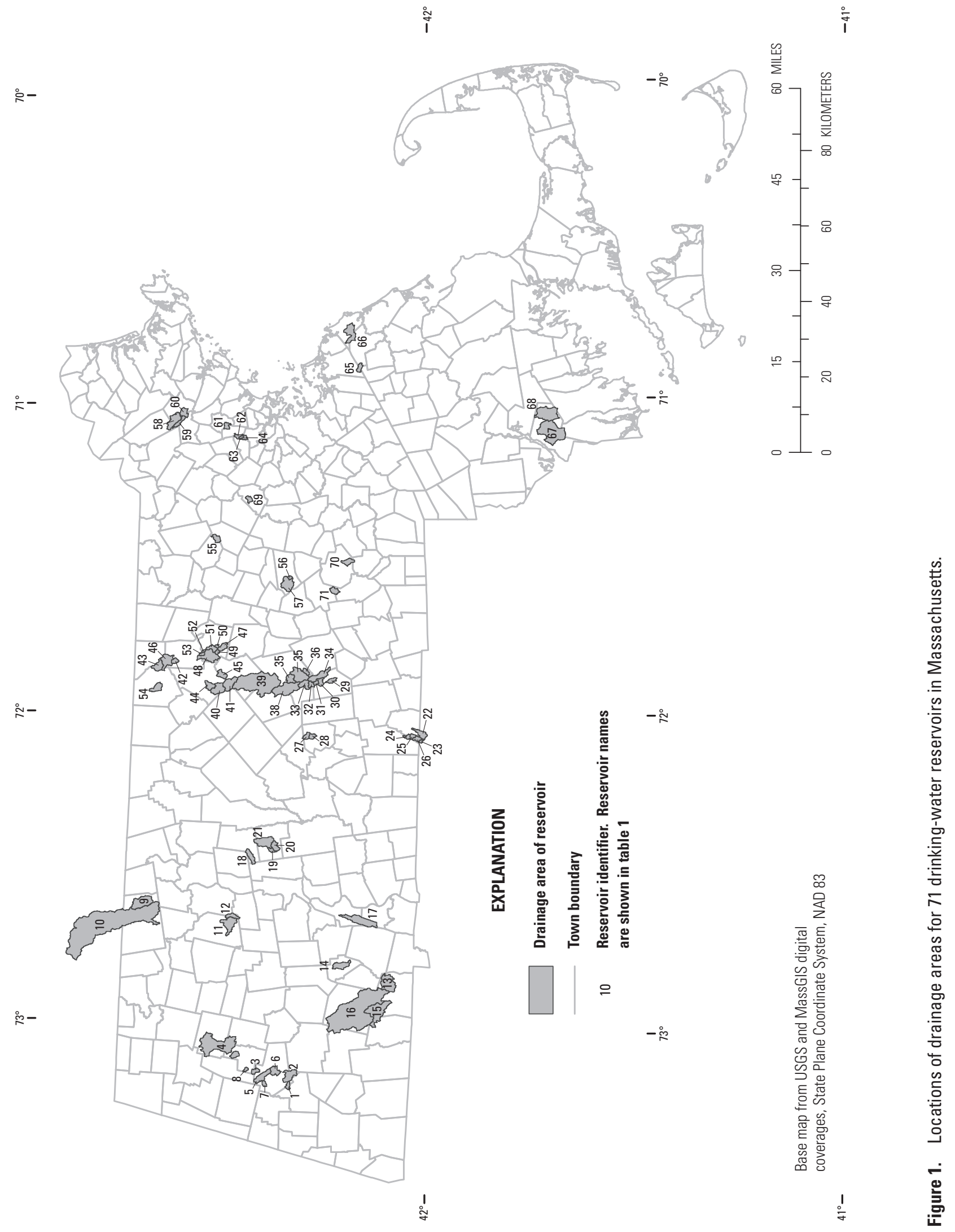
Table 1. Massachusetts drinking-water-supply systems and associated reservoirs included in this study.

[ID, identifier; SUASCO, Sudbury, Assabet, Concord; DPW, Department of Public Works]

\begin{tabular}{|c|c|c|c|}
\hline Water supplier & Reservoir name & Reservoir ID & Drainage basin \\
\hline \multirow[t]{4}{*}{ Amherst Water Department } & Atkins Reservoir & 18 & Connecticut \\
\hline & Hill Reservoir & 21 & Connecticut \\
\hline & Hawley Reservoir & 19 & Connecticut \\
\hline & Amethyst Brook Intake & 20 & Connecticut \\
\hline Ashburnham/Winchendon Joint Water Board & Upper Naukeag Lake & 54 & Millers \\
\hline Concord Water Department & Nagog Pond & 55 & SUASCO \\
\hline \multirow[t]{3}{*}{ Danvers Water Department } & Emerson Brook Pond & 58 & Ipswich \\
\hline & Middleton Pond & 60 & Ipswich \\
\hline & Swan Pond & 59 & Ipswich \\
\hline \multirow[t]{2}{*}{ Fall River Water Department } & North Watuppa Reservoir & 67 & Narragansett Bay \\
\hline & Copicut Reservoir & 68 & Buzzards Bay \\
\hline \multirow[t]{7}{*}{ Fitchburg Water Department } & Bickford Pond & 41 & Chicopee \\
\hline & Mare Meadow Reservoir & 40 & Chicopee \\
\hline & Meetinghouse Reservoir & 44 & Nashua \\
\hline & Wachusett Lake & 45 & Nashua \\
\hline & Scott Reservoir & 42 & Nashua \\
\hline & Fitchburg Reservoir & 43 & Nashua \\
\hline & Lovell Reservoir & 46 & Nashua \\
\hline \multirow[t]{2}{*}{ Greenfield Water Department } & Green River & 10 & Deerfield \\
\hline & Leyden Glen Reservoir & 9 & Deerfield \\
\hline Hingham/Hull (Aquarian Water Company) & Accord Pond & 65 & Boston Harbor \\
\hline Hinsdale Water Department & Belmont Reservoir & 8 & Housatonic \\
\hline \multirow[t]{2}{*}{ Lee Water Department } & Schoolhouse Reservoir & 2 & Housatonic \\
\hline & Upper (Leahey) Reservoir & 1 & Housatonic \\
\hline Leicester (Cherry Valley and Rochdale Water) & Henshaw Pond & 29 & French \\
\hline \multirow[t]{7}{*}{ Leominster DPW-Water Division } & Distributing Reservoir & 51 & Nashua \\
\hline & Morse Reservoir & 50 & Nashua \\
\hline & Haynes Reservoir & 49 & Nashua \\
\hline & Simonds Pond & 52 & Nashua \\
\hline & Goodfellow Pond & 53 & Nashua \\
\hline & Notown Reservoir & 48 & Nashua \\
\hline & Fall Brook Reservoir & 47 & Nashua \\
\hline Lincoln Water Department & Flints Pond (Sandy Pond) & 69 & Charles \\
\hline \multirow[t]{2}{*}{ Marlborough DPW-Water and Sewer Division } & Millham Reservoir & 57 & SUASCO \\
\hline & Williams Lake & 56 & SUASCO \\
\hline Milford Water Company & Echo Lake & 70 & Charles \\
\hline
\end{tabular}


Refinement and Evaluation of the Massachusetts Firm-Yield Estimator Model Version 2.0

Table 1. Massachusetts drinking-water-supply systems and associated reservoirs included in this study.-Continued

[ID, identifier; SUASCO, Sudbury, Assabet, Concord; DPW, Department of Public Works]

\begin{tabular}{|c|c|c|c|}
\hline Water supplier & Reservoir name & Reservoir ID & Drainage basin \\
\hline \multirow[t]{2}{*}{ North Brookfield Water Department } & Doane Pond & 28 & Chicopee \\
\hline & Horse Pond & 27 & Chicopee \\
\hline \multirow[t]{5}{*}{ Pittsfield Water Department } & Ashley Lake/Lower Ashley Intake & 3 & Housatonic \\
\hline & Farnham Reservoir & 7 & Housatonic \\
\hline & Sandwash Reservoir & 6 & Housatonic \\
\hline & Upper Sackett Reservoir & 5 & Housatonic \\
\hline & Cleveland Reservoir & 4 & Housatonic \\
\hline Scituate Water Department & Tack Factory Pond & 66 & South Coastal \\
\hline \multirow[t]{5}{*}{ Southbridge Water Department } & Hatchet Pond & 23 & Quinebaug \\
\hline & Hatchet Brook Reservoir \#3 & 24 & Quinebaug \\
\hline & Hatchet Brook Reservoir \#4 & 25 & Quinebaug \\
\hline & Hatchet Brook Reservoir \#5 & 26 & Quinebaug \\
\hline & Cohasse Brook Reservoir & 22 & Quinebaug \\
\hline \multirow[t]{2}{*}{ Springfield Water Department } & Borden Brook Reservoir & 15 & Westfield \\
\hline & Cobble Mountain Reservoir & 16 & Westfield \\
\hline \multirow[t]{2}{*}{ South Deerfield Water Supply District } & Roaring Brook Reservoir & 11 & Connecticut \\
\hline & Whately Reservoir & 12 & Connecticut \\
\hline Wakefield Water Department & Crystal Lake & 61 & North Coastal \\
\hline Westborough Water Department & Sandra Pond & 71 & SUASCO \\
\hline \multirow[t]{2}{*}{ Westfield Water Department } & Granville Reservoir & 13 & Westfield \\
\hline & Montgomery Reservoir & 14 & Westfield \\
\hline West Springfield Water Department & Bearhole Reservoir & 17 & Westfield \\
\hline \multirow[t]{3}{*}{ Winchester Water Department } & Middle Reservoir & 63 & Mystic \\
\hline & North Reservoir & 62 & Mystic \\
\hline & South Reservoir & 64 & Mystic \\
\hline \multirow[t]{10}{*}{ Worcester Water Department } & Holden Reservoir \#1 & 35 & Blackstone \\
\hline & Holden Reservoir \#2 & 36 & Blackstone \\
\hline & Kettle Brook Reservoir \#1 & 30 & Blackstone \\
\hline & Kettle Brook Reservoir \#2 & 36 & Blackstone \\
\hline & Kettle Brook Reservoir \#3 & 32 & Blackstone \\
\hline & Kettle Brook Reservoir \#4 & 33 & Blackstone \\
\hline & Lynde Brook Reservoir & 34 & Blackstone \\
\hline & Kendall Reservoir & 37 & Nashua \\
\hline & Pine Hill Reservoir & 38 & Nashua \\
\hline & Quinapoxet Reservoir & 39 & Nashua \\
\hline
\end{tabular}




\section{Surface-Water Inflows}

Surface-water inflow volumes are generally orders of magnitude larger than the volumes of climatic waterbalance components or groundwater contributions, making them one of the most important inputs in the determination of the firm yield. A recently developed method makes it possible to estimate daily streamflows for most locations in Massachusetts. The Sustainable Yield Estimator (SYE) (Archfield, 2010) application was used to estimate daily streamflow for all the reservoirs in this study. The SYE uses a regression-based method to develop a daily-flow-duration curve for an ungaged stream for the period of October 1, 1960 to September 30, 2004. The flow-duration curve is then transformed into a daily time series by equating the quantiles of the ungaged flow-duration curve to a flow-duration curve of a gaged index stream during the same time period. This method is similar to the method of streamflow estimation used in previous Massachusetts firm-yield studies (Waldron and Archfield, 2006) and the MassDEP FYE guidance document; however, the SYE tool employs new regression techniques and more robust index-streamflow-selection methods. For single-reservoir systems, basin characteristics used in the SYE regression equations were calculated for the entire reservoir watershed, excluding the reservoir area (table 2). For reservoirs in series along a stream, the areas of all upstream reservoir watersheds were excluded from the calculation of downstream reservoir basin characteristics.

\section{Precipitation and Evaporation}

Records of daily precipitation and temperature were obtained from 226 National Climatic Data Center (NCDC) climate stations in and around Massachusetts (fig. 2). There were 157 stations with daily precipitation records ranging from 1 to 44 years during the simulation period. Stations had varying amounts of missing data throughout the record, and no station had a complete daily record for the entire 44 years. In order to obtain a complete daily precipitation record at each reservoir, inverse-squared distance weighted interpolation of gaged precipitation stations was used. Although this method can result in an underestimation of precipitation variability, this method has been shown to perform as well or better than using data from the nearest precipitation station to estimate daily precipitation (Kruizinga and Yperlaan, 1978). For each day of the simulation period, inverse-squared distance interpolation was performed using the three nearest gaged stations within 15 miles (mi) that had recorded precipitation data for that day. Because each meteorological station had records of differing lengths, the set of stations used in the daily interpolation of precipitation may have varied from day to day for a given FYE reservoir site.

The interpolated daily precipitation was cross-validated using observed data from 31 long-term precipitation gages.
Gages with over 40 years of data were chosen for validation, as well as two gages in the western part of Massachusetts where gages with 40 -year records were not available. For each validation gage, inverse-squared distance weighted interpolation of the surrounding gages was used to produce a time series of daily precipitation for the gaged location. The estimated daily precipitation at each gaged station was then compared to the observed data, and the Nash Sutcliffe Efficiency (NSE) was calculated (Nash and Sutcliffe, 1970). The NSE is a commonly used indicator to assess the fit of a hydrologic model. An NSE value of 1 indicates a perfect fit between observed and modeled values. An NSE of 0 means that the modeled data has the same amount of error as just using the mean of the observed data. A negative NSE indicates that the mean of the observed data provides a better estimate than the modeled data. The NSE for the 31 validation gages ranged from -0.16 to 0.87 with a median of 0.68 (table 3 ).

Only one site, located at the Worcester Regional Airport, had a NSE of less than zero. This site is located in a sparsely gaged area. Only one other gage falls within $5 \mathrm{mi}$ of the Worcester site, and that gage has data from only October 1960 to November 1962. The second nearest gage to the Worcester site is $9.8 \mathrm{mi}$ away. An analysis of the interpolated time series for the Worcester site shows that NSE calculated from October 1960 to November 1962 is 0.58 , whereas the NSE for the remaining period is -0.23 , indicating that interpolation results may be more unreliable when gaged sites are farther than $5 \mathrm{mi}$ away. Other validation sites that performed poorly are located in areas of the State that are not densely gaged, indicating that estimated daily precipitation may be less accurate for reservoirs in sparsely gaged areas of Massachusetts.

Daily precipitation time series were validated using observed precipitation at two reservoirs. Daily precipitation measurements were available for Atkins Reservoir for March 1, 1994 to September 30, 2004, and for Echo Lake for October 1, 1960 to September 30, 1967. The interpolated precipitation time series showed good agreement with observed precipitation for both Atkins Reservoir and Echo Lake, with NSEs of 0.94 and 0.75 , respectively (table 3 ).

Evaporation from lakes depends on meteorological factors as well as physical characteristics of the lake, but is generally equivalent to estimates of potential evapotranspiration. Daily evaporation from each reservoir was estimated using Hargreaves' equation for potential evapotranspiration (Hargreaves and Samni, 1982). This is a temperature-based method requiring daily average temperatures and daily temperature ranges. Daily minimum and maximum temperatures were interpolated from the NCDC gaged climate stations. There were 94 climate stations with daily minimum and maximum temperature records for periods ranging from 1 to 44 years during the simulation period. Temperatures were interpolated using inverse-squared distance for each reservoir in the same manner as for precipitation. 
Table 2. Reservoir basin characteristics and index streamgages in Massachusetts used in the Sustainable Yield Estimator.

$\left[\mathrm{mi}^{2}\right.$, square miles; ft, feet; in, inches; ${ }^{\circ} \mathrm{C}$, degrees Celsius; SYE, Sustainable Yield Estimator; DPW, Department of Public Works; X- and Y-coordinates in

\begin{tabular}{|c|c|c|c|c|c|}
\hline Water supplier & Reservoir name & $\begin{array}{c}\text { Drain- } \\
\text { age area, } \\
\text { excluding } \\
\text { surface area } \\
\left(\mathrm{mi}^{2}\right)\end{array}$ & $\begin{array}{c}\text { Mean } \\
\text { basin } \\
\text { elevation } \\
\text { (ft) }\end{array}$ & $\begin{array}{l}\text { Average } \\
\text { annual pre- } \\
\text { cipitation } \\
\text { (in) }\end{array}$ & $\begin{array}{c}\text { Percentage } \\
\text { of basin } \\
\text { that is open } \\
\text { water }\end{array}$ \\
\hline \multirow{4}{*}{ Amherst Water Department } & Atkins Reservoir & 1.72 & 735.28 & 48.18 & 0.18 \\
\hline & Hill Reservoir & 4.05 & $1,052.11$ & 49.01 & 0.00 \\
\hline & Hawley Reservoir & 1.50 & 877.33 & 48.58 & 0.07 \\
\hline & Amethyst Brook Intake & 0.63 & 641.52 & 48.17 & 0.00 \\
\hline Ashburnham/Winchendon Joint Water Board & Upper Naukeag Lake & 1.37 & $1,197.61$ & 49.15 & 0.25 \\
\hline Concord Water Department & Nagog Pond & 0.77 & 255.53 & 46.46 & 0.75 \\
\hline \multirow[t]{3}{*}{ Danvers Water Department } & Emerson Brook Pond & 3.26 & 118.93 & 46.56 & 2.88 \\
\hline & Middleton Pond & 1.32 & 118.73 & 46.81 & 14.21 \\
\hline & Swan Pond & 1.19 & 127.79 & 46.63 & 1.94 \\
\hline \multirow{2}{*}{ Fall River Water Department } & North Watuppa Reservoir & 8.47 & 167.87 & 48.95 & 1.87 \\
\hline & Copicut Reservoir & 5.66 & 193.97 & 49.14 & 0.02 \\
\hline \multirow[t]{7}{*}{ Fitchburg Water Department } & Bickford Pond & 3.03 & $1,226.32$ & 49.90 & 0.11 \\
\hline & Mare Meadow Reservoir & 2.64 & $1,165.12$ & 48.81 & 0.05 \\
\hline & Meetinghouse Reservoir & 1.32 & $1,124.74$ & 48.79 & 0.00 \\
\hline & Wachusett Lake & 1.32 & $1,155.20$ & 50.08 & 13.64 \\
\hline & Scott Reservoir & 0.73 & $1,006.59$ & 48.81 & 0.07 \\
\hline & Fitchburg Reservoir & 1.90 & $1,161.41$ & 49.36 & 0.16 \\
\hline & Lovell Reservoir & 3.20 & $1,012.32$ & 48.99 & 0.17 \\
\hline \multirow[t]{2}{*}{ Greenfield Water Department } & Green River & 52.06 & $1,239.88$ & 51.06 & 0.55 \\
\hline & Leyden Glen Reservoir & 5.16 & 950.26 & 49.52 & 0.10 \\
\hline Hingham/Hull (Aquarian Water Company) & Accord Pond & 0.79 & 161.96 & 46.75 & 0.62 \\
\hline Hinsdale Water Department & Belmont Reservoir & 0.37 & $1,899.15$ & 49.92 & 4.57 \\
\hline \multirow[t]{2}{*}{ Lee Water Department } & Schoolhouse Reservoir & 2.94 & $1,889.41$ & 53.39 & 1.60 \\
\hline & Upper (Leahey) Reservoir & 0.63 & $1,808.39$ & 51.21 & 0.00 \\
\hline Leicester (Cherry Valley and Rochdale Water) & Henshaw Pond & 0.88 & 865.80 & 48.92 & 6.20 \\
\hline \multirow[t]{7}{*}{ Leominster DPW-Water Division } & Distributing Reservoir & 1.14 & 810.73 & 49.56 & 0.00 \\
\hline & Morse Reservoir & 0.28 & 802.11 & 49.65 & 0.00 \\
\hline & Haynes Reservoir & 0.34 & 889.48 & 50.09 & 0.00 \\
\hline & Simonds Pond & 0.18 & 806.71 & 49.11 & 0.00 \\
\hline & Goodfellow Pond & 0.41 & 776.33 & 48.99 & 0.20 \\
\hline & Notown Reservoir & 3.96 & 853.66 & 49.43 & 0.00 \\
\hline & Fall Brook Reservoir & 1.21 & 814.81 & 49.98 & 0.18 \\
\hline Lincoln Water Department & Flints Pond (Sandy Pond) & 0.55 & 267.40 & 46.99 & 0.01 \\
\hline \multirow[t]{2}{*}{ Marlborough DPW-Water and Sewer Division } & Millham Reservoir & 3.40 & 367.12 & 47.95 & 0.23 \\
\hline & Williams Lake & 0.26 & 459.58 & 47.90 & 7.02 \\
\hline
\end{tabular}


Massachusetts State Plane meters; Elevation determined from the U.S. Geological Survey 30-meter National Elevation Dataset]

\begin{tabular}{|c|c|c|c|c|c|c|c|c|}
\hline $\begin{array}{l}\text { X-coor- } \\
\text { dinate } \\
\text { of the } \\
\text { basin } \\
\text { outlet }\end{array}$ & $\begin{array}{l}\text { Y-coor- } \\
\text { dinate } \\
\text { of the } \\
\text { basin } \\
\text { outlet }\end{array}$ & $\begin{array}{c}\text { Average } \\
\text { maximum } \\
\text { monthly } \\
\text { temperature } \\
\left({ }^{\circ} \mathrm{C}\right)\end{array}$ & $\begin{array}{l}\text { Percent- } \\
\text { age of } \\
\text { basin } \\
\text { that is } \\
\text { wetland }\end{array}$ & $\begin{array}{c}\text { Percentage } \\
\text { of basin that } \\
\text { is underlain } \\
\text { by sand and } \\
\text { gravel }\end{array}$ & $\begin{array}{l}\text { X-coor- } \\
\text { dinate } \\
\text { of the } \\
\text { basin } \\
\text { centroid }\end{array}$ & $\begin{array}{l}\text { Y-coor- } \\
\text { dinate } \\
\text { of the } \\
\text { basin } \\
\text { centroid }\end{array}$ & $\begin{array}{c}\text { SYE } \\
\text { selected } \\
\text { index-gage } \\
\text { station } \\
\text { number }\end{array}$ & SYE selected index-gage station name \\
\hline 118777 & 908834 & 13.81 & 1.48 & 8.83 & 120426 & 909179 & 01174565 & West Branch Swift River near Shutesbury, Mass. \\
\hline 122433 & 903927 & 13.29 & 7.74 & 14.17 & 124269 & 905494 & 01174900 & Cadwell Creek near Belchertown, Mass. \\
\hline 122301 & 903193 & 13.49 & 1.68 & 8.63 & 123102 & 902723 & 01174901 & Cadwell Creek near Belchertown, Mass. \\
\hline 121496 & 903761 & 14.03 & 1.18 & 30.51 & 122101 & 903622 & 01174902 & Cadwell Creek near Belchertown, Mass. \\
\hline 164340 & 934333 & 13.04 & 5.92 & 0.00 & 165210 & 934113 & 01161500 & Tarbell Brook near Winchendon, Mass. \\
\hline 205467 & 917705 & 15.06 & 2.03 & 7.67 & 204811 & 918242 & 01097300 & Nashoba Brook near Acton, Mass. \\
\hline 238199 & 928219 & 14.97 & 21.55 & 2.17 & 236141 & 929547 & 01100700 & East Meadow River near Haverhill, Mass. \\
\hline 239406 & 927213 & 14.98 & 16.30 & 5.23 & 238221 & 926859 & 01100701 & East Meadow River near Haverhill, Mass. \\
\hline 237151 & 926546 & 14.95 & 21.33 & 1.81 & 235723 & 928121 & 01100702 & East Meadow River near Haverhill, Mass. \\
\hline 231755 & 825696 & 14.97 & 10.01 & 22.23 & 233089 & 829278 & 01106000 & Adamsville Brook at Adamsville, R.I. \\
\hline 238117 & 827911 & 14.96 & 15.38 & 12.46 & 237949 & 830452 & 01106001 & Adamsville Brook at Adamsville, R.I. \\
\hline 164479 & 914888 & 12.79 & 7.04 & 0.00 & 166284 & 914773 & 01095220 & Stillwater River near Sterling, Mass. \\
\hline 164177 & 916252 & 12.98 & 10.05 & 0.20 & 164906 & 917830 & 01095221 & Stillwater River near Sterling, Mass. \\
\hline 166748 & 920465 & 13.18 & 6.83 & 0.00 & 165767 & 920066 & 01095222 & Stillwater River near Sterling, Mass. \\
\hline 168481 & 918229 & 12.93 & 2.93 & 31.48 & 168711 & 916854 & 01095223 & Stillwater River near Sterling, Mass. \\
\hline 173121 & 929106 & 13.57 & 0.93 & 0.00 & 172300 & 929338 & 01096000 & Squannacook River near West Groton, Mass. \\
\hline 171935 & 932770 & 13.16 & 3.16 & 0.87 & 171006 & 933726 & 01096001 & Squannacook River near West Groton, Mass. \\
\hline 173661 & 929522 & 13.50 & 3.25 & 0.43 & 172349 & 931483 & 01096002 & Squannacook River near West Groton, Mass. \\
\hline 108115 & 933534 & 12.60 & 1.56 & 3.46 & 102185 & 947478 & 01170100 & Green River near Colrain, Mass. \\
\hline 108452 & 935080 & 13.16 & 2.01 & 0.00 & 108237 & 938076 & 01170101 & Green River near Colrain, Mass. \\
\hline 250453 & 880610 & 14.97 & 9.09 & 41.52 & 250131 & 880232 & 01105600 & Old Swamp River near South Weymouth, Mass. \\
\hline 64426 & 910317 & 11.44 & 2.07 & 0.00 & 63828 & 910493 & 01174566 & West Branch Swift River near Shutesbury, Mass. \\
\hline 60722 & 900541 & 11.37 & 9.91 & 0.00 & 62276 & 898907 & 01174567 & West Branch Swift River near Shutesbury, Mass. \\
\hline 58665 & 898849 & 11.61 & 1.42 & 0.00 & 59529 & 899287 & 01174568 & West Branch Swift River near Shutesbury, Mass. \\
\hline 167375 & 886553 & 13.54 & 10.39 & 2.31 & 167098 & 887692 & 01175670 & Sevenmile River near Spencer, Mass. \\
\hline 176425 & 919701 & 13.97 & 1.80 & 11.01 & 175631 & 919403 & 01095224 & Stillwater River near Sterling, Mass. \\
\hline 176232 & 918736 & 14.26 & 0.00 & 11.80 & 176215 & 918123 & 01095225 & Stillwater River near Sterling, Mass. \\
\hline 175076 & 918327 & 13.78 & 2.76 & 2.11 & 174682 & 918051 & 01095226 & Stillwater River near Sterling, Mass. \\
\hline 175060 & 921964 & 13.98 & 0.91 & 0.00 & 174887 & 921729 & 01095227 & Stillwater River near Sterling, Mass. \\
\hline 174545 & 921838 & 13.97 & 5.28 & 30.40 & 174160 & 922081 & 01095228 & Stillwater River near Sterling, Mass. \\
\hline 173915 & 921424 & 13.85 & 7.39 & 5.25 & 173605 & 920156 & 01095229 & Stillwater River near Sterling, Mass. \\
\hline 176961 & 916339 & 14.12 & 5.14 & 0.00 & 176023 & 916544 & 01095230 & Stillwater River near Sterling, Mass. \\
\hline 215274 & 908829 & 15.04 & 10.62 & 5.41 & 215247 & 909592 & 01097301 & Nashoba Brook near Acton, Mass. \\
\hline 190750 & 899448 & 14.76 & 9.40 & 12.95 & 192695 & 899418 & 01095231 & Stillwater River near Sterling, Mass. \\
\hline 194093 & 898424 & 14.62 & 1.36 & 0.00 & 194406 & 898748 & 01097302 & Nashoba Brook near Acton, Mass. \\
\hline
\end{tabular}


Table 2. Reservoir basin characteristics and index streamgages in Massachusetts used in the Sustainable Yield Estimator.-Continued $\left[\mathrm{mi}^{2}\right.$, square miles; ft, feet; in, inches; ${ }^{\circ} \mathrm{C}$, degrees Celsius; SYE, Sustainable Yield Estimator; DPW, Department of Public Works; X- and Y-coordinates in

\begin{tabular}{|c|c|c|c|c|c|}
\hline Water supplier & Reservoir name & $\begin{array}{c}\text { Drain- } \\
\text { age area, } \\
\text { excluding } \\
\text { surface area } \\
\left(\mathrm{mi}^{2}\right)\end{array}$ & $\begin{array}{c}\text { Mean } \\
\text { basin } \\
\text { elevation } \\
\text { (ft) }\end{array}$ & $\begin{array}{l}\text { Average } \\
\text { annual pre- } \\
\text { cipitation } \\
\text { (in) }\end{array}$ & $\begin{array}{c}\text { Percentage } \\
\text { of basin } \\
\text { that is open } \\
\text { water }\end{array}$ \\
\hline Milford Water Company & Echo Lake & 1.26 & 434.41 & 48.04 & 0.00 \\
\hline \multirow[t]{2}{*}{ North Brookfield Water Department } & Doane Pond & 0.75 & 971.14 & 48.56 & 1.45 \\
\hline & Horse Pond & 0.79 & $1,008.25$ & 48.58 & 0.25 \\
\hline \multirow[t]{5}{*}{ Pittsfield Water Department } & Ashley Lake/Lower Ashley Intake & 2.43 & $1,876.28$ & 51.47 & 0.01 \\
\hline & Farnham Reservoir & 2.93 & $1,871.90$ & 52.10 & 3.30 \\
\hline & Sandwash Reservoir & 1.67 & $1,956.55$ & 53.44 & 0.83 \\
\hline & Upper Sackett Reservoir & 0.86 & $1,793.27$ & 50.80 & 0.00 \\
\hline & Cleveland Reservoir & 13.74 & $1,900.26$ & 52.30 & 0.05 \\
\hline Scituate Water Department & Main Reservoir & 3.50 & 91.99 & 49.42 & 0.85 \\
\hline \multirow[t]{5}{*}{ Southbridge Water Department } & Hatchet Pond & 0.18 & 909.38 & 51.55 & 4.88 \\
\hline & Hatchet Brook Reservoir \#3 & 0.58 & 769.80 & 51.10 & 0.00 \\
\hline & Hatchet Brook Reservoir \#4 & 0.54 & 767.57 & 51.41 & 0.04 \\
\hline & Hatchet Brook Reservoir \#5 & 0.88 & 876.36 & 51.60 & 0.31 \\
\hline & Cohasse Brook Reservoir & 1.76 & 783.94 & 51.61 & 0.00 \\
\hline \multirow[t]{2}{*}{ Springfield Water Department } & Borden Brook Reservoir & 7.64 & 156.94 & 53.83 & 0.95 \\
\hline & Cobble Mountain Reservoir & 35.88 & 179.39 & 54.74 & 1.03 \\
\hline \multirow[t]{2}{*}{ South Deerfield Water Supply District } & Roaring Brook Reservoir & 3.97 & 847.42 & 49.32 & 0.00 \\
\hline & Whately Reservoir & 1.22 & 690.84 & 47.68 & 0.29 \\
\hline Wakefield Water Department & Crystal Lake & 0.74 & 144.64 & 47.83 & 0.00 \\
\hline Westborough Water Department & Sandra Pond & 1.10 & 512.74 & 48.13 & 0.67 \\
\hline \multirow[t]{2}{*}{ Westfield Water Department } & Granville Reservoir & 5.14 & 169.87 & 51.79 & 0.01 \\
\hline & Montgomery Reservoir & 2.47 & 169.42 & 51.64 & 0.92 \\
\hline West Springfield Water Department & Bearhole Reservoir & 5.51 & 156.94 & 47.83 & 9.65 \\
\hline \multirow[t]{3}{*}{ Winchester Water Department } & Middle Reservoir & 0.22 & 188.78 & 47.89 & 0.00 \\
\hline & North Reservoir & 0.53 & 181.41 & 47.91 & 0.63 \\
\hline & South Reservoir & 0.35 & 196.31 & 47.85 & 0.00 \\
\hline \multirow[t]{10}{*}{ Worcester Water Department } & Holden Reservoir \#1 & 4.31 & 988.16 & 49.10 & 0.26 \\
\hline & Holden Reservoir \#2 & 0.66 & 870.25 & 48.89 & 10.78 \\
\hline & Kettle Brook Reservoir \#1 & 1.05 & 997.81 & 49.09 & 0.19 \\
\hline & Kettle Brook Reservoir \#2 & 0.46 & $1,056.53$ & 49.21 & 9.47 \\
\hline & Kettle Brook Reservoir \#3 & 0.68 & $1,102.12$ & 49.32 & 0.00 \\
\hline & Kettle Brook Reservoir \#4 & 1.65 & $1,155.72$ & 49.32 & 0.03 \\
\hline & Lynde Brook Reservoir & 2.99 & 955.96 & 48.90 & 0.90 \\
\hline & Kendall Reservoir & 1.42 & 962.25 & 49.11 & 0.00 \\
\hline & Pine Hill Reservoir & 6.20 & $1,051.33$ & 48.91 & 1.65 \\
\hline & Quinapoxet Reservoir & 19.23 & 978.06 & 49.24 & 1.16 \\
\hline
\end{tabular}


Massachusetts State Plane meters; Elevation determined from the U.S. Geological Survey 30-meter National Elevation Dataset]

\begin{tabular}{|c|c|c|c|c|c|c|c|c|}
\hline $\begin{array}{l}\text { X-coor- } \\
\text { dinate } \\
\text { of the } \\
\text { basin } \\
\text { outlet }\end{array}$ & $\begin{array}{l}\text { Y-coor- } \\
\text { dinate } \\
\text { of the } \\
\text { basin } \\
\text { outlet }\end{array}$ & $\begin{array}{c}\text { Average } \\
\text { maximum } \\
\text { monthly } \\
\text { temperature } \\
\left({ }^{\circ} \mathrm{C}\right)\end{array}$ & $\begin{array}{l}\text { Percent- } \\
\text { age of } \\
\text { basin } \\
\text { that is } \\
\text { wetland }\end{array}$ & $\begin{array}{c}\text { Percentage } \\
\text { of basin that } \\
\text { is underlain } \\
\text { by sand and } \\
\text { gravel }\end{array}$ & $\begin{array}{l}\text { X-coor- } \\
\text { dinate } \\
\text { of the } \\
\text { basin } \\
\text { centroid }\end{array}$ & $\begin{array}{l}\text { Y-coor- } \\
\text { dinate } \\
\text { of the } \\
\text { basin } \\
\text { centroid }\end{array}$ & $\begin{array}{c}\text { SYE } \\
\text { selected } \\
\text { index-gage } \\
\text { station } \\
\text { number }\end{array}$ & SYE selected index-gage station name \\
\hline 199336 & 882420 & 14.89 & 7.43 & 0.00 & 198632 & 883339 & 01111500 & Branch River at Forestdale, R.I. \\
\hline 152832 & 892890 & 13.37 & 6.03 & 0.00 & 152294 & 892837 & 01175671 & Sevenmile River near Spencer, Mass. \\
\hline 152716 & 893521 & 13.26 & 9.44 & 0.00 & 152338 & 894330 & 01175672 & Sevenmile River near Spencer, Mass. \\
\hline 61112 & 907556 & 11.49 & 7.71 & 0.00 & 63253 & 904598 & 01174569 & West Branch Swift River near Shutesbury, Mass. \\
\hline 59624 & 905955 & 11.40 & 12.11 & 0.00 & 60066 & 905596 & 01174570 & West Branch Swift River near Shutesbury, Mass. \\
\hline 62375 & 903170 & 11.26 & 17.66 & 0.00 & 63447 & 902808 & 01174571 & West Branch Swift River near Shutesbury, Mass. \\
\hline 62981 & 908976 & 11.67 & 5.35 & 0.00 & 63448 & 907981 & 01174572 & West Branch Swift River near Shutesbury, Mass. \\
\hline 67217 & 914454 & 11.40 & 11.65 & 2.15 & 67782 & 913389 & 01331400 & Dry Brook near Adams, Mass. \\
\hline 261649 & 882089 & 14.90 & 19.31 & 8.34 & 259381 & 882706 & 01105731 & Indian Head River at Hanover, Mass. \\
\hline 151250 & 864151 & 13.60 & 0.13 & 0.00 & 150964 & 863976 & 01126600 & Blackwell Brook near Brooklyn, Conn. \\
\hline 152645 & 867506 & 13.96 & 1.11 & 1.60 & 152153 & 867618 & 01126601 & Blackwell Brook near Brooklyn, Conn. \\
\hline 152344 & 866907 & 13.87 & 3.18 & 1.17 & 152234 & 866210 & 01126602 & Blackwell Brook near Brooklyn, Conn. \\
\hline 151867 & 866016 & 13.67 & 4.57 & 1.42 & 151592 & 864861 & 01126603 & Blackwell Brook near Brooklyn, Conn. \\
\hline 154253 & 866456 & 13.92 & 4.32 & 0.00 & 152957 & 863965 & 01126604 & Blackwell Brook near Brooklyn, Conn. \\
\hline 81048 & 876485 & 12.78 & 6.82 & 1.94 & 79241 & 875630 & 01187300 & Hubbard River near West Hartland, Conn. \\
\hline 84932 & 875556 & 12.90 & 5.31 & 2.00 & 79243 & 881286 & 01187400 & Valley Brook near West Hartland, Conn. \\
\hline 104207 & 913954 & 13.57 & 3.48 & 3.30 & 102484 & 914911 & 01169900 & South River near Conway, Mass. \\
\hline 105145 & 913574 & 13.91 & 1.40 & 0.00 & 104560 & 914117 & 01169901 & South River near Conway, Mass. \\
\hline 235056 & 916292 & 14.88 & 4.44 & 14.16 & 234734 & 915498 & 01097303 & Nashoba Brook near Acton, Mass. \\
\hline 191280 & 888045 & 14.59 & 5.81 & 21.87 & 190928 & 886932 & 01111501 & Branch River at Forestdale, R.I. \\
\hline 87851 & 871847 & 13.65 & 0.93 & 4.68 & 86724 & 872716 & 01187401 & Valley Brook near West Hartland, Conn. \\
\hline 91646 & 883065 & 13.30 & 8.82 & 6.54 & 91509 & 885160 & 01174573 & West Branch Swift River near Shutesbury, Mass. \\
\hline 102251 & 875730 & 15.08 & 5.77 & 26.76 & 103356 & 879967 & 01171800 & Bassett Brook near North Hampton, Mass. \\
\hline 231596 & 911210 & 14.84 & 2.84 & 0.00 & 231654 & 911686 & 01097304 & Nashoba Brook near Acton, Mass. \\
\hline 231403 & 912841 & 14.85 & 2.99 & 8.29 & 231998 & 912548 & 01097305 & Nashoba Brook near Acton, Mass. \\
\hline 231434 & 910191 & 14.86 & 2.13 & 0.00 & 231549 & 910787 & 01097306 & Nashoba Brook near Acton, Mass. \\
\hline 169341 & 895131 & 13.32 & 3.82 & 5.85 & 168502 & 896269 & 01175673 & Sevenmile River near Spencer, Mass. \\
\hline 169817 & 894051 & 13.70 & 1.15 & 6.58 & 169470 & 894531 & 01175674 & Sevenmile River near Spencer, Mass. \\
\hline 167666 & 889650 & 13.21 & 5.77 & 0.00 & 166741 & 890617 & 01175675 & Sevenmile River near Spencer, Mass. \\
\hline 167001 & 891321 & 13.08 & 2.72 & 0.00 & 166530 & 891878 & 01175676 & Sevenmile River near Spencer, Mass. \\
\hline 166337 & 892458 & 13.00 & 8.00 & 0.00 & 166039 & 893177 & 01175677 & Sevenmile River near Spencer, Mass. \\
\hline 165489 & 893916 & 12.92 & 10.67 & 0.00 & 166060 & 894897 & 01175678 & Sevenmile River near Spencer, Mass. \\
\hline 169080 & 888876 & 13.39 & 8.28 & 0.00 & 168367 & 890969 & 01175679 & Sevenmile River near Spencer, Mass. \\
\hline 167957 & 899596 & 13.43 & 2.22 & 5.65 & 167553 & 898601 & 01095232 & Stillwater River near Sterling, Mass. \\
\hline 166626 & 900134 & 13.09 & 8.44 & 0.00 & 164905 & 899773 & 01095233 & Stillwater River near Sterling, Mass. \\
\hline 168780 & 904242 & 13.33 & 9.26 & 9.04 & 166454 & 907298 & 01095234 & Stillwater River near Sterling, Mass. \\
\hline
\end{tabular}


$43^{\circ}-$

$\boldsymbol{A}$

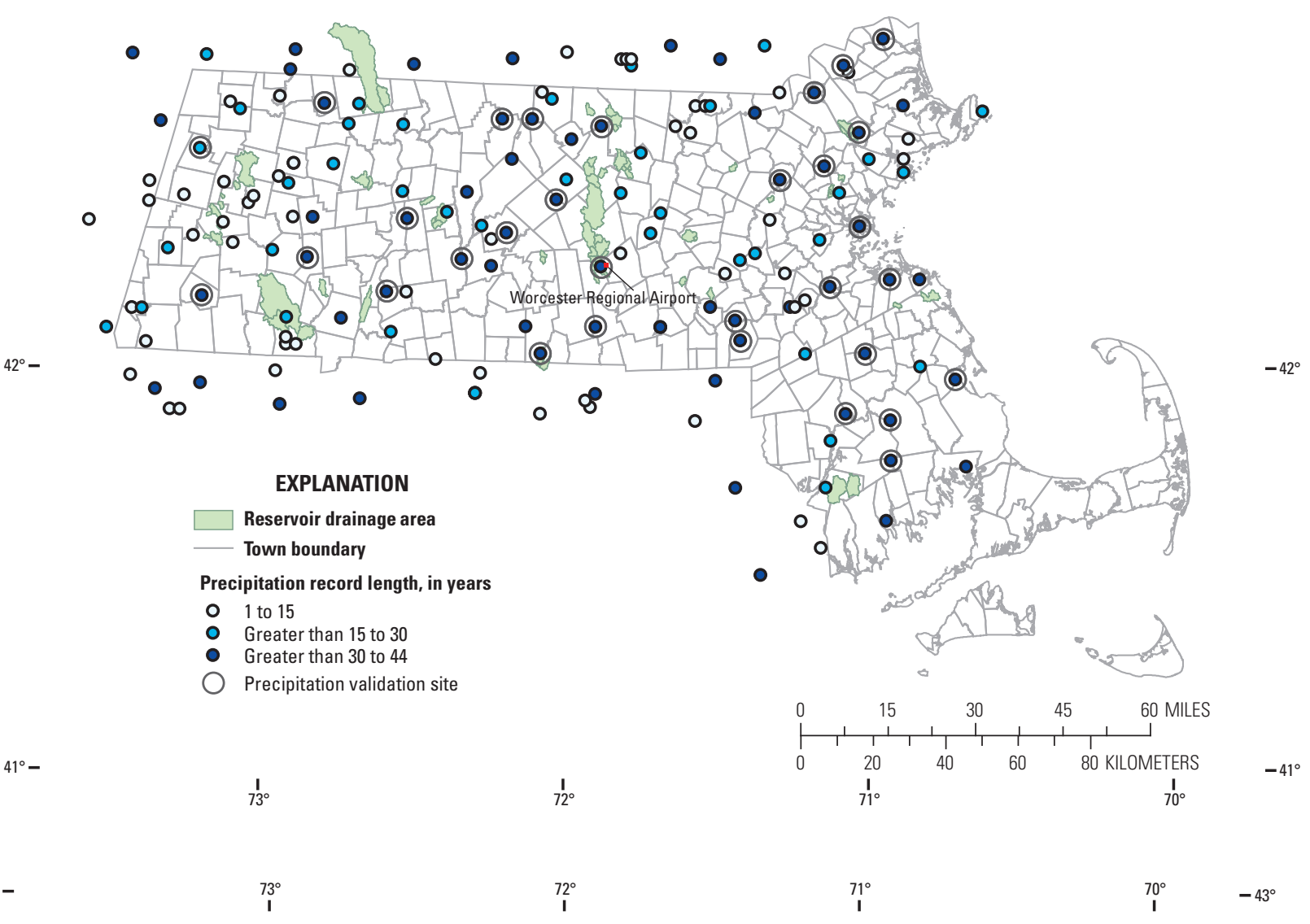

B

$42^{\circ}-$

$\circ$

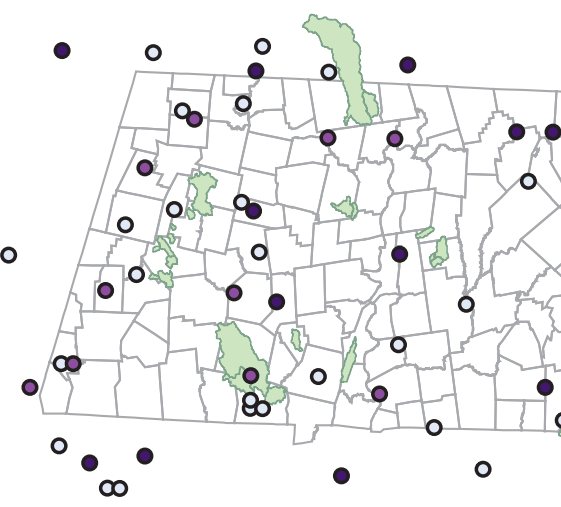

EXPLANATION

$\square$ Reservoir drainage area
- Town boundary
Temperature record length, in years
O 1 through 15
- 16 through 30
- 31 through 44

$41^{\circ}-$

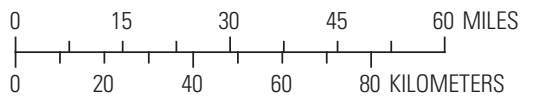

$-41^{\circ}$

$$
73^{1}
$$

$71^{\circ}$

Figure 2. Location of reservoirs and record length at $A$, precipitation stations and validations sites, and $B$, meteorological stations in Massachusetts and vicinity. 
Table 3. Nash-Sutcliffe Efficiency of daily precipitation at 33 gaged validation sites in Massachusetts.

[NCDC COOP ID, National Climatic Data Center Cooperative Station identifier; --, no data]

\begin{tabular}{lcc}
\hline \multicolumn{1}{c}{ Precipitation-gage location } & $\begin{array}{c}\text { NCDC COOP } \\
\text { ID }\end{array}$ & $\begin{array}{c}\text { Nash-Sutcliffe } \\
\text { Efficiency }\end{array}$ \\
\hline Amherst & 190120 & 0.67 \\
Ashburnham & 190190 & 0.52 \\
Barre Falls Dam & 190408 & 0.81 \\
Bedford & 190535 & 0.53 \\
Belchertown & 190562 & 0.86 \\
Birch Hill Dam & 190666 & 0.84 \\
East Milton/Blue Hill & 190736 & 0.49 \\
Logan Airport, Boston & 190770 & 0.66 \\
Brockton & 190860 & 0.51 \\
Buffumville Lake, Charlton & 190998 & 0.77 \\
Franklin & 192997 & 0.84 \\
Hardwick & 193401 & 0.84 \\
Haverhill & 193505 & 0.60 \\
Heath & 193549 & 0.73 \\
Hingham & 193624 & 0.70 \\
Holyoke & 193702 & 0.57 \\
Knightville Dam, Huntington & 193985 & 0.75 \\
Lanesboro & 194075 & 0.65 \\
Lawrence & 194105 & 0.36 \\
Middleboro & 194711 & 0.70 \\
Middleton & 194744 & 0.79 \\
Newburyport & 195285 & 0.42 \\
Plymouth/Kingston & 196486 & 0.69 \\
Reading & 196783 & 0.68 \\
Rochester & 196938 & 0.67 \\
Southbridge & 197627 & 0.87 \\
Taunton & 198367 & 0.37 \\
Tully Lake, Royalston & 198573 & 0.83 \\
West Medway & 199316 & 0.81 \\
West Otis & 199371 & 0.65 \\
Worcester Regional Airport & 199923 & -0.16 \\
Atkins Reservoir, Amherst & --94 \\
\hline & & 0.75 \\
\hline
\end{tabular}

\section{Groundwater Contributions}

Where a reservoir is in contact with an aquifer, groundwater may flow into or out of the reservoir depending on aquifer water table and reservoir stage. When reservoir stage falls because water is being used to meet demand, the decrease in stage will cause water from the aquifer to flow into the reservoir until the reservoir stage and aquifer water table regain equilibrium. In contrast, when reservoir stage rises, water from the reservoir may flow into the aquifer. During an extended drawdown period, groundwater inflows can potentially provide enough additional water to the reservoir storage to maintain a yield that would otherwise lead to failure.

Rorabaugh (1964) developed an analytical solution to the groundwater-flow equation for the case of one-dimensional flow in a finite-width aquifer bounded by a stream. Archfield and Carlson (2006) adapted these equations to estimate the groundwater contribution to reservoir storage due to timevarying changes in reservoir stage and applied the equations in the FYE model. When reservoir stage changes from one time step to the next, groundwater contributions are estimated as a flow rate per unit length (Archfield and Carlson, 2006, equations 3, 4, and 5). The flow rate is then multiplied by a scaling factor, $L$, which is the estimated length of reservoir shoreline in contact with the aquifer, in order to determine the total volume of groundwater flow per day. In the analysis by Archfield and Carlson (2006), $L$ was parameterized as a constant, calculated as the perimeter in contact with sand and gravel for a reservoir at full pool. In reality, a reservoir may be in contact with an aquifer along any part of its bottom surface area, not just the perimeter, and the area that is in contact with the aquifer will decrease as the reservoir storage volume is depleted. Reservoirs at very low storage levels have a much smaller surface area that could be interacting with the aquifer and thus, the scaling parameter should vary with this surface area.

Parameterizing $L$ as a constant results in overestimation of groundwater contributions during extreme reservoir drawdown periods when reservoir storage is low. Archfield and Carlson (2006) found that the overestimation of groundwater flows at low reservoir-storage levels led to numerical instabilities in the groundwater-flow equations, making groundwater-flow estimates for some reservoirs impossible. In order to better characterize groundwater contributions for this study, the scaling parameter $L$ was allowed to vary on the basis of the reservoir stage. At full pool, $L$ was defined in the same manner as in Archfield and Carlson (2006), as the length of the perimeter that intersected sand and gravel deposits. This was determined in a GIS by intersecting the full-pool contour line with a digital data layer of sand and gravel deposits (Office of Geographic and Environmental Information, 2004). In order to estimate the value of $L$ when the reservoir stage was lower than full pool, the surface area of the reservoir at each contour interval was overlaid on the digital data layer of sand and gravel deposits. The value of $L$ at each contour was estimated by decreasing the value of $L$ at full pool in proportion to the decrease in the area which intersected sand and gravel at that contour level. 


\section{Applications of the Firm-Yield Estimator Model}

Firm yields were determined for 38 reservoir systems in Massachusetts. Of these, 23 were single-reservoir systems, and 15 were multiple-reservoir systems. Reservoirs whose firm yield had been previously determined were re-evaluated using updated methods and data. Previous firm-yield studies in Massachusetts (Waldron and Archfield, 2006; and Archfield and Carlson, 2006) used a monthly time step, which can cause an overestimation of the firm yield (Fennessey, 1995). The inclusion of daily streamflows and meteorological data as well as a refined method for estimating groundwater flows in this study resulted in firm-yield estimates that differ from those found in Waldron and Archfield (2006).

Firm-yield estimates for both single- and multiplereservoir systems are shown in table 4. Water-use data from Annual Statistical Reports provided by MassDEP for the years 2000 to 2004 indicate that 32 out of the 38 reservoir systems are currently operating below their firm yield or marginally at their firm yield. Because year-to-year usage in a system can vary, systems in which average usage was within 10 percent of the firm yield may be operating within the firm yield in some years but above the firm yield in other years. These reservoirs were listed as marginally operating within the firm yield.

\section{Application of the Firm-Yield Estimator Model to a Single-Reservoir System}

Single-reservoir systems consist of one reservoir that does not receive water from any other reservoir. Inflows to a single-reservoir system consist of streamflow into the reservoir from the surrounding watershed, precipitation that falls directly on the reservoir surface, and groundwater inflows. Outflows from a single-reservoir system are evapotranspiration, groundwater outflows, water withdrawals for public water supply or other uses, and releases from the reservoir for downstream flow requirements. Firm yields for a single-reservoir system are computed by successively solving the water-balance equation for the reservoir over the 44-year simulation period, increasing the yield each time by a minimum of 0.001 million gallons (Mgal). The highest yield that can be used without the reservoir failing for one day is the firm yield.

Echo Lake in Hopkinton, Massachusetts, currently augments the amount of available water in the reservoir by a diversion from the Charles River (not shown in fig. 1) approximately 2.5 mi below the Echo Lake Dam, at the crossing of Dilla Street in Milford, Mass. Water from the river at this point can be pumped back into the reservoir. The maximum pumping rate for the diversion is 3 million gallons per day $(\mathrm{Mgal} / \mathrm{d})$, and pumping primarily occurs during March through May (Metcalf and Eddy, 1997). In order to simulate this unique situation, streamflow for the length of the Charles River from the diversion point up to the Echo Lake Dam was estimated using SYE. The FYE model was modified for this reservoir only, such that a maximum of $3 \mathrm{Mgal} / \mathrm{d}$ of streamflow from the Charles River and from Echo Lake spillage during the previous simulated time step was added back into Echo Lake storage at the end of each simulation time step during the months of March through May. Including the Charles River diversion in the simulation of Echo Lake increased the firm yield by 35 percent, from $0.83 \mathrm{Mgal} / \mathrm{d}$ without the diversion to $1.12 \mathrm{Mgal} / \mathrm{d}$ including the diversion. A previously estimated firm yield for this lake, calculated using a reservoir capacity of $384 \mathrm{Mgal}$ obtained from historical documents and without the Charles River skimming, is $0.56 \mathrm{Mgal} / \mathrm{d}$ (Metcalf and Eddy, 1997). The current study used a reservoir capacity of 461.6 Mgal based on volume estimates from current bathymetric measurements of the reservoir.

Main Reservoir in Scituate is simulated as a singlereservoir system, although water is actually transported from Main Reservoir to Old Oaken Bucket Pond by way of First Herring Brook (not shown in fig. 1) before being pumped to the water-treatment facility. Because of excessive aquaticplant growth, bathymetric measurements could not be made in Old Oaken Bucket Pond. Because the stage-storage relation could not be determined for this reservoir, the firm yield could not be estimated. On the basis of the relative sizes of these two reservoirs, most of the available water for this system comes from Main Reservoir. Because storage in Old Oaken Bucket Pond was not considered for this analysis, the firm yield for this system represents a conservative estimate.

\section{Application of the Firm-Yield Estimator Model to a Multiple-Reservoir System}

Of the 71 reservoirs included in this study, 48 are in reservoir systems in which multiple reservoirs are hydrologically connected. Reservoirs may be configured in a variety of ways and can transfer water either through uncontrolled spills from an upstream reservoir that is at full pool into a river or open channel that transports water by gravity into a downstream reservoir, or by pumping water from one reservoir to another. Reservoir-configuration diagrams for newly surveyed reservoirs in this study are included in appendix 3. Configurations for previously studied reservoir systems are included in Archfield and Waldron (2006). Because individual reservoirs in a system have different storage capacities, they will not necessarily deplete and refill at the same rates. The optimal firm yield for a multiple-reservoir system depends on the time-varying withdrawal operations of each individual reservoir in the system. Estimating the optimal firm yield for a multiple-reservoir system requires complex optimization methods which are beyond the scope of this study. However, an approximation of a multiple-reservoir-system firm yield can be made by assuming that all reservoirs are operating at their individual maximum yield. 
Table 4. Firm-yield estimates and 2000-2004 water usage for 38 reservoir systems in Massachusetts. Marlborough, Leominster, Wakefield, and Worcester have water-supply connections with the Massachusetts Water Resources Authority in addition to reservoirs listed here.

[Actual average annual usage refers to the period 2000-2004. Reservoirs with usage that was within 10 percent of the firm yield are considered marginally meeting their firm yield; Mgal/d, million gallons per day; DPW, Department of Public Works]

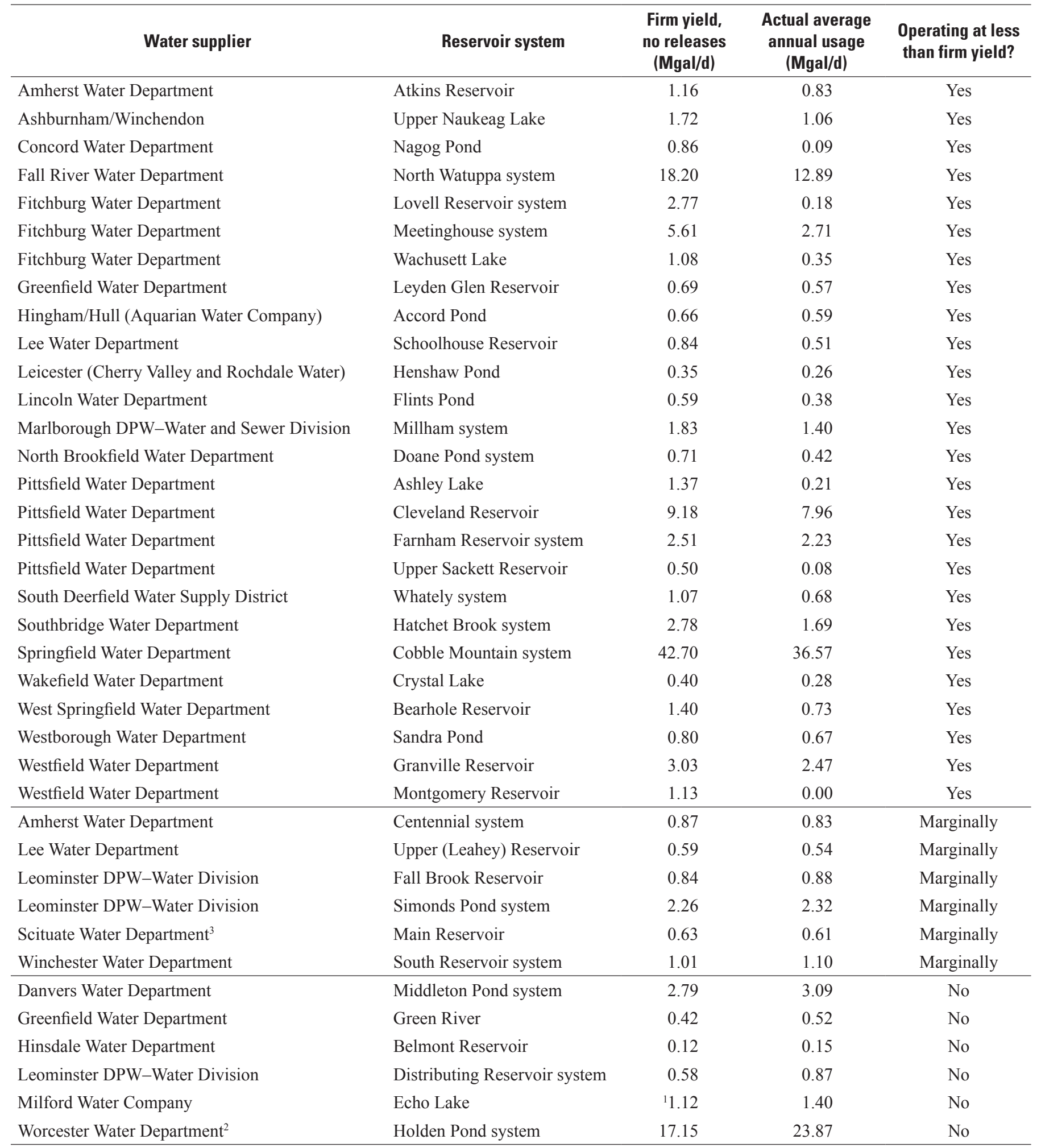

${ }^{1}$ Firm yield for Echo Lake includes water from the Charles River diversion.

${ }^{2}$ Firm yield for the Worcester reservoir system is subject to additional uncertainty due to complex water routing between reservoirs.

${ }^{3}$ Firm yield for Scituate does not include Old Oaken Bucket Pond. 
The algorithm for calculating the firm yield of a multiple-reservoir system depends on its configuration and the method of water transport between reservoirs (fig. 3). For two reservoirs connected by either a river or open channel, water released from the upstream reservoir in order to meet demand, as well as any uncontrolled spills and controlled releases from the reservoir, is transported to the downstream reservoir. For two reservoirs that transport water through pumping, only the volume of water that is pumped from the first reservoir is added as a water input to the second reservoir, and uncontrolled spills and controlled releases are lost from the system. The firm-yield model first estimates the firm yield of the uppermost reservoir, which does not receive water from any other reservoirs, in the same manner as a single-reservoir system. The spillage volume, $Q_{s p i}$ and the yield volume, $Q_{y i}$ are calculated for each simulation day and saved by the model. After the simulation for the first reservoir is finished, the model begins the simulation for the second reservoir and includes the inputs from the previous reservoir in the water balance. If the reservoirs are connected by pumping, the daily-yield volume from the previous reservoir is added as an additional inflow into the water-balance equation of the second reservoir. For reservoirs connected by gravity, the daily-yield volume, $Q_{y i}$, and the daily uncontrolled spill volume, $Q_{s p i}$, are added as additional inflows into the water balance of the second reservoir. The maximum pump capacity may be set by the user. If the usage exceeds the pump capacity on a given day, only the maximum pump-capacity volume is transferred to the second reservoir, and the excess water remains in storage in the first reservoir. For systems with more than two reservoirs, firm yields of reservoirs that contribute water to any other reservoir are estimated before the receiving reservoir. The system firm yield is the firm yield calculated at the terminal reservoir after accounting for inflows from other reservoirs in the system.

The FYE is most appropriate for estimating yields of relatively simple multiple-reservoir configurations and reservoir operations. Because the model calculates the yield of each reservoir in the system sequentially, reservoir configurations in which water is routed through a reservoir more than once cannot be handled by the FYE. Only one reservoir system in the study had a configuration that could not be adequately modeled by the FYE. The Worcester system consists of 10 individual reservoirs with Holden Reservoir \#1 as the terminal reservoir. Water is pumped into Holden Reservoir \#1 from Lynde Brook Reservoir and gravity-fed by Kendall Reservoir. Water from Holden Reservoir \#1 may be pumped either directly to the filtration plant or into Holden Reservoir \#2 for storage, then back into Holden Reservoir \#1. Because Holden Reservoir \#1 both receives water from and releases water to Holden Reservoir \#2, it cannot be modeled by the sequential algorithm used in the FYE. In order to estimate the firm yield for this system, Holden Reservoirs \#1 and \#2 were modeled as one reservoir with the combined reservoir characteristics of both individual reservoirs. Because of the operational complexities of this system, the firm yield estimated by the FYE is less certain than in simpler reservoir systems.

\section{Firm-Yield Estimate Uncertainty and Sensitivity to Model Inputs}

Uncertainty in firm yields determined using the FYE comes from many sources, including uncertainty in input data - such as daily streamflow, evaporation, precipitation, and stage-storage relations - and uncertainty associated with the estimation of groundwater-flow contributions. For reservoir systems that are operating at or near their estimated firm yield, the uncertainty associated with the firm-yield estimates could result in reservoirs exceeding their true firm yield. If the firmyield estimate is higher than the true value of the firm yield, reservoirs risk failing if they are operating near their firmyield estimate.

An analytical method to examine the effects of the combined uncertainty from all of the input data errors has not been determined for the FYE; however, the sensitivity of the model to individual sources of uncertainty can be examined. Daily streamflow volumes are generally many times greater than groundwater contributions and direct precipitation or evaporation from the reservoir surface. Therefore, relative uncertainties in the daily streamflow time series have the potential to contribute the greatest amount of uncertainty in the water balance equation and the resulting firm-yield estimate. The storage capacity of the reservoir is also important in estimating the firm yield. Reservoir volume and stage/storage relations are calculated from bathymetric-survey data. There is little guidance available regarding the number of bathymetric-data points needed to accurately assess reservoir capacity and the manner in which errors in this calculation might affect the firm-yield estimates. A final source of uncertainty in the firm-yield estimates comes from the equations used to estimate groundwater contributions to the water balance. These equations have not been extensively tested for use in reservoir simulation models, and parameter estimates for these equations are uncertain.

\section{Sensitivity of Firm-Yield Estimates to Errors in Daily Streamflow}

In order to investigate the potential sensitivity of the FYE to input streamflow errors, a series of Monte Carlo simulations were performed in which the daily-streamflow inputs were perturbed by a random-error term during the calculation of the firm yield for a particular reservoir. The range of firm-yield estimates obtained after many simulations is representative of the amount of uncertainty that can be expected in the firm yield due to errors in the input streamflow time series. 

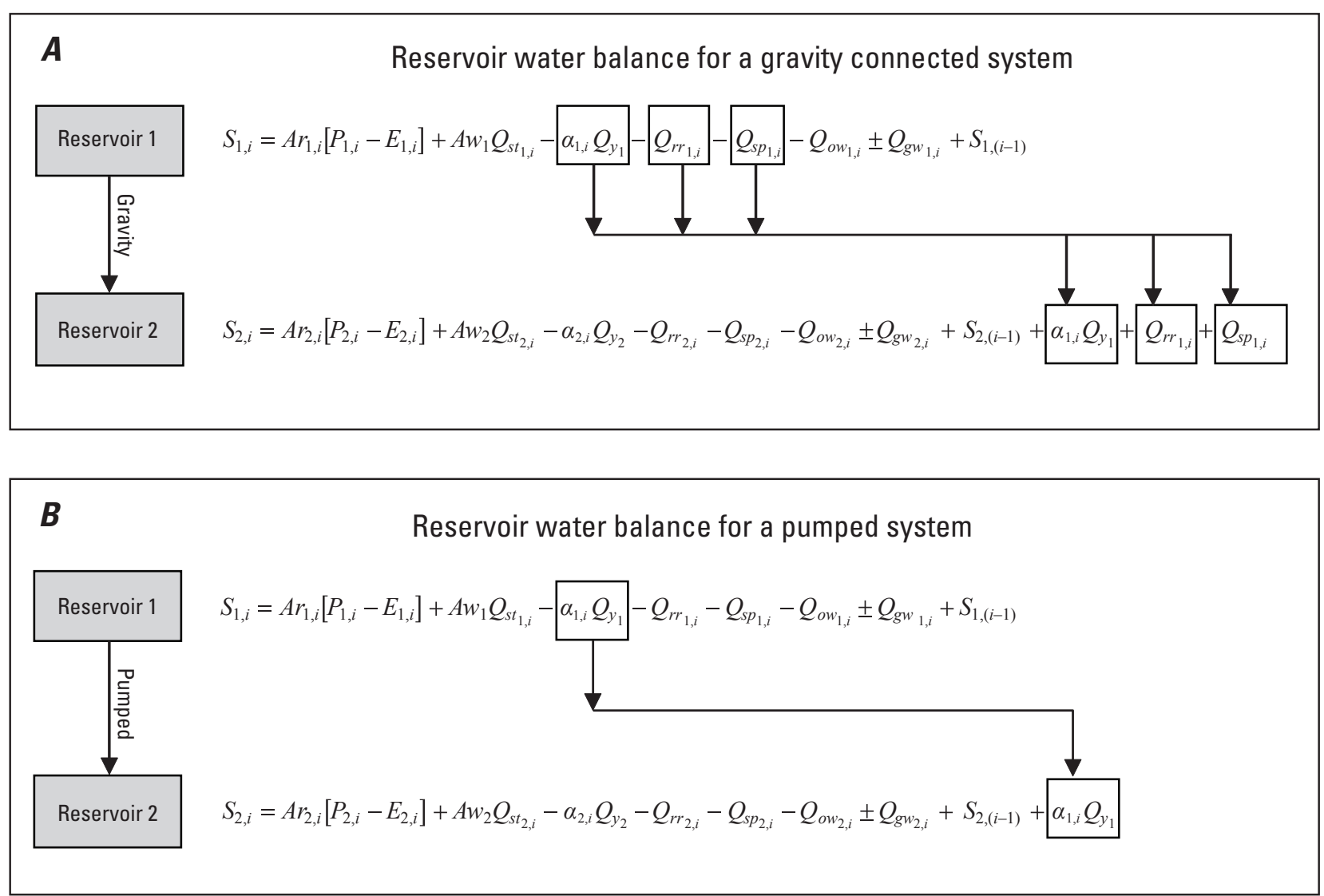

\footnotetext{
$i=$ daily simulation time step

$S_{i}=$ volume of water in usable storage for the current day, in million gallons

$A r_{i}=$ area of the reservoir surface, in square miles

$P_{i}=$ precipitation, in miles

$E_{i}=$ evaporation from the reservoir surface, in miles

$A w_{i}=$ reservoir drainage area, in square miles

$Q_{s t_{i}}=$ streamflow per unit drainage area, in miles

$\alpha_{i}=$ peak-usage factor, dimensionless
}

\section{EXPLANATION}

Subscripts 1 and 2 identify the reservoirs

Figure 3. Water balances for a system of reservoirs in which $A$, water is transported by gravity and $B$, water is pumped from Reservoir 1 to Reservoir 2. 
In order for the result of a Monte Carlo sensitivity analysis to be meaningful, the errors added to the streamflow in the simulations must accurately characterize the uncertainty in the input data. Uncertainty associated with SYE streamflow estimates is an area of ongoing research and is beyond the scope of this project (Archfield and others, 2010). However, as a first attempt at characterizing the uncertainty associated with FYE streamflow inputs, the structure of the randomly generated Monte Carlo error terms was based on the relative errors from a SYE cross-validation dataset. SYE-generated daily streamflows were computed for 18 U.S. Geological Survey (USGS) gaged sites and compared to the observed streamflow values (Archfield and others, 2010). The percent difference between the SYE predicted daily flows and the observed flows from these 18 sites was calculated for each day as

$$
P D=\left(Q_{e s t}-Q_{o b s}\right) / Q_{e s t},
$$

where

$P D \quad$ is the percent difference between the SYE
predicted streamflow and observed
streamflow,

For each of the gages, the exceedence probability of each of the daily flows also was calculated. For each exceedence probability, the mean percent difference between observed and estimated flows across all 18 gaged sites was calculated as well as the standard deviation of the percent differences.

Figure 4 shows that for streamflows with exceedence probabilities up to about 0.6 , the mean percent difference between observed and predicted is near zero, and the standard deviation of the percent differences is fairly constant, indicating that flows in this range are relatively unbiased but contain some amount of random error. For low streamflows with higher exceedence probabilities, the mean percent difference between observed and predicted deviates from zero, and the mean standard deviation of the differences increases, indicating a potential bias and greater uncertainty in the low daily streamflows. Since low flows generally occur during drought periods, underprediction of streamflows during these times could lead to underprediction of the firm-yield estimate.

In order to construct a random-error term for inclusion in the Monte Carlo simulations, the percent difference between observed and predicted daily streamflows was assumed to be normally distributed. The mean and standard deviation of the sample distribution for the percent difference between observed and predicted flows were estimated from figure 4 . For simulated flows with exceedence probabilities less than 0.6 , the error term was generated from a Gaussian distribution with a mean of zero and a standard deviation of 0.3 . In order to capture the greater uncertainty in the low flows, simulated streamflows with exceedence probabilities greater than 0.95 were generated from a Gaussian distribution with a mean of -0.75 and a standard deviation of 1 . Streamflows with exceedence probabilities of 0.6 to 0.95 were generated from a Gaussian distribution with a mean of -0.25 and a standard deviation of 0.5 . Although the percent differences between observed and predicted streamflows are large at higher exceedence probabilities, the percent difference represents a very small volume of water, because flows at the 0.95 exceedence probability and above are generally very low for the stream gages in the cross-validation dataset. Random percent errors generated for each day of the simulation were applied to the daily streamflows as

$$
Q^{*}=Q_{e s t}-\left(\operatorname{Err}_{i} * Q_{e s t}\right)
$$

where

$$
\begin{gathered}
Q^{*} \quad \begin{array}{c}
\text { is the daily streamflow modified by random } \\
\text { error term, }
\end{array} \\
Q_{e s t} \quad \begin{array}{l}
\text { is the daily-streamflow volume estimated by } \\
\text { the SYE, and }
\end{array} \\
E r r_{i} \quad \text { is the randomly generated error term. }
\end{gathered}
$$

Monte Carlo simulations with 500 repetitions were run for 22 reservoirs in the study, and the distribution of the resulting firm yields for each reservoir is shown in table 5 . Firm yields generated by the Monte Carlo simulations in the selected reservoir were somewhat higher than the original firm-yield estimates. This is due to the underprediction bias in the lowest streamflows. When this bias is corrected in the Monte Carlo simulations, firm yields increase. The mean firm yield generated by the Monte Carlo simulations ranged from 0.6 to 10.6 percent higher than original firm-yield estimates across all 22 reservoirs, indicating that firm yields computed by the model are potentially underestimated by this amount and therefore represent a conservative estimate of the firm yield (table 5).

The mean percent change in firm yield was used to compare the sensitivity behaviors of different reservoirs. Mean percent change in firm yield was calculated as the percent difference between the initial firm-yield estimate and the mean firm-yield estimate generated by the Monte Carlo simulation. The mean percent changes in firm yields were highest in reservoirs with low storage ratios, defined as the reservoir capacity divided by the mean annual streamflow. This indicates that these low-storage reservoirs are more sensitive to errors in the streamflow input data than reservoirs with high storage ratios (fig. 5).

It is important to note that because the sample distribution used to generate the random errors in the Monte Carlo simulations was based on the average behavior at 18 gaged sites, it may not accurately characterize the errors in streamflow input data at all FYE reservoirs. The errors in the streamflows for any particular reservoir may have more or less bias or variability than the average relative errors at the gaged sites and, therefore, would have a different range of firm-yield estimates. In addition, this method does not account for autocorrelation of the daily relative errors in streamflows. Further study regarding uncertainty in the SYE tool is needed for a more precise sensitivity analysis of the effects of streamflow input data on the firm yield. 


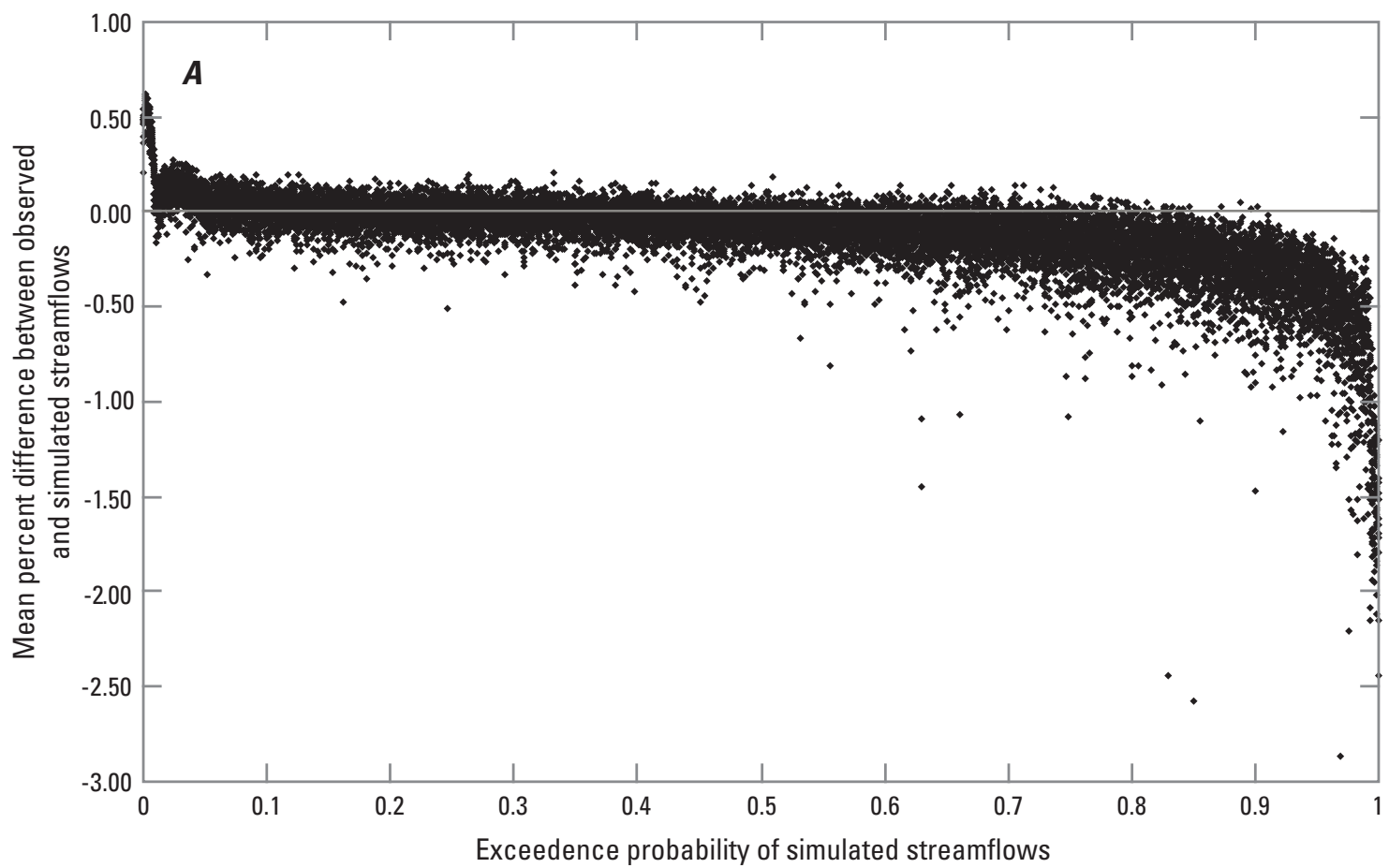

Overprediction of daily flows

Underprediction of daily flows

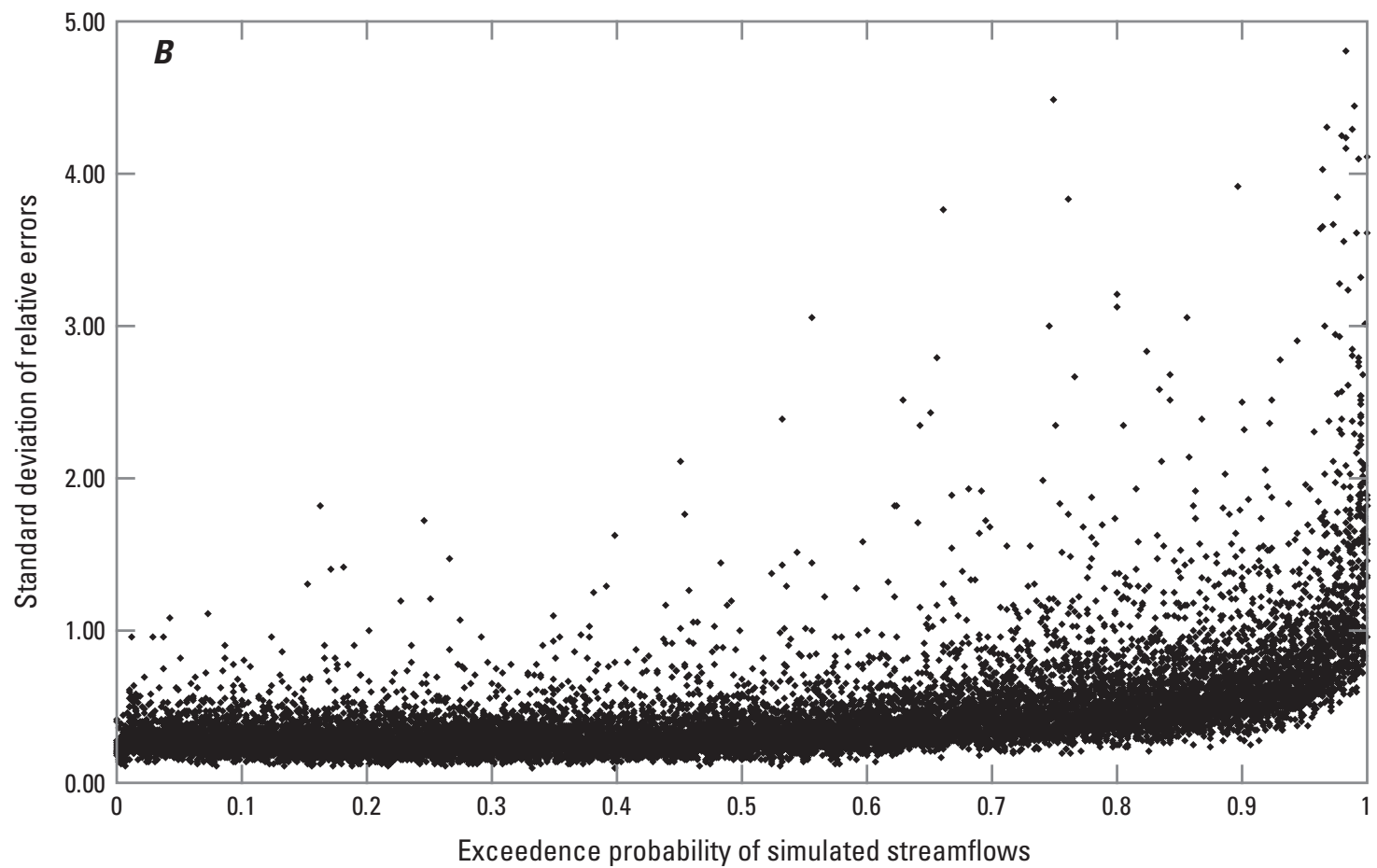

Figure 4. $A$, Mean percent difference and $B$, standard deviation of the percent difference of daily streamflows generated by the Sustainable Yield Estimator at 18 U.S. Geological Survey gaged sites in Massachusetts. 
Table 5. Distribution of firm yields resulting from 500 Monte Carlo simulations at selected reservoirs in Massachusetts. For the purpose of this analysis, all reservoirs were simulated as single-reservoir systems. Marlborough, Leominster, Wakefield, and Worcester have water-supply connections with the Massachusetts Water Resources Authority in addition to reservoirs listed here.

[Mgal/d, million gallons per day]

\begin{tabular}{|c|c|c|c|c|c|}
\hline Reservoir name & $\begin{array}{l}\text { Firm-yield } \\
\text { estimate } \\
\text { (Mgal/d) }\end{array}$ & $\begin{array}{c}\text { Mean firm-yield } \\
\text { estimate from Monte } \\
\text { Carlo simulations } \\
\text { (Mgal/d) }\end{array}$ & $\begin{array}{l}\text { Standard deviation } \\
\text { of Monte Carlo firm- } \\
\text { yield estimates }\end{array}$ & $\begin{array}{c}\text { Highest Monte Carlo } \\
\text { firm-yield estimate } \\
\text { (Mgal/d) }\end{array}$ & $\begin{array}{c}\text { Lowest Monte Carlo } \\
\text { firm-yield estimate } \\
\text { (Mgal/d) }\end{array}$ \\
\hline Ashley Lake & 1.37 & 1.42 & 0.02 & 1.47 & 1.36 \\
\hline Atkins Reservoir & 1.16 & 1.28 & 0.02 & 1.33 & 1.23 \\
\hline Belmont Reservoir & 0.12 & 0.12 & 0.00 & 0.13 & 0.12 \\
\hline Bickford Reservoir & 1.91 & 1.96 & 0.02 & 2.01 & 1.91 \\
\hline Cleveland Reservoir & 9.18 & 9.56 & 0.10 & 9.86 & 9.24 \\
\hline Cohasse Reservoir & 1.16 & 1.19 & 0.02 & 1.24 & 1.13 \\
\hline Copicut Reservoir & 7.30 & 7.41 & 0.05 & 7.56 & 7.25 \\
\hline Emerson Brook Reservoir & 1.22 & 1.27 & 0.02 & 1.32 & 1.23 \\
\hline Fall Brook Reservoir & 0.84 & 0.86 & 0.01 & 0.89 & 0.82 \\
\hline Farnham Reservoir & 1.62 & 1.69 & 0.02 & 1.74 & 1.63 \\
\hline Fitchburg Reservoir & 1.22 & 1.24 & 0.01 & 1.27 & 1.20 \\
\hline Flints Pond & 0.59 & 0.59 & 0.00 & 0.61 & 0.58 \\
\hline Granville Reservoir & 3.03 & 3.32 & 0.13 & 3.59 & 3.07 \\
\hline Hatchet Pond & 0.21 & 0.21 & 0.00 & 0.22 & 0.21 \\
\hline Henshaw Pond & 0.35 & 0.37 & 0.01 & 0.38 & 0.35 \\
\hline Horse Pond & 0.50 & 0.51 & 0.01 & 0.53 & 0.49 \\
\hline Leyden Glen Reservoir & 0.69 & 0.75 & 0.01 & 0.79 & 0.71 \\
\hline Quinapoxet Reservoir & 4.21 & 4.46 & 0.03 & 4.57 & 4.38 \\
\hline Sandwash Reservoir & 0.89 & 0.93 & 0.01 & 0.97 & 0.89 \\
\hline Schoolhouse Reservoir & 0.84 & 0.91 & 0.01 & 0.94 & 0.88 \\
\hline Upper Sackett Reservoir & 0.50 & 0.51 & 0.01 & 0.53 & 0.48 \\
\hline Wachusett Lake & 1.08 & 1.10 & 0.02 & 1.15 & 1.06 \\
\hline
\end{tabular}

\section{Sensitivity of the Firm-Yield Model to Bathymetric-Map Accuracy}

Water-depth measurements of a reservoir are necessary to determine the maximum storage capacity of the reservoir and the relation between reservoir stage and storage. Reservoirdepth measurements are interpolated using a GIS to create a continuous bathymetric map from which lake volume can be determined. Because the firm yield of a reservoir is dependent upon the storage capacity, it is important that the number of water-depth measurements collected is adequate to characterize this input variable to the FYE model.

Bathymetric measurements are generally made along parallel or gridded straight-line transects of the lake. The spacing of transect intervals is important because the density of water-depth measurements affects the accuracy of the computer-generated bathymetric maps and computed storage capacity. Although it is beneficial to have as many measurement points as possible, very high density transect intervals may be prohibitively time consuming and expensive to obtain, especially for large reservoirs. However, if the spacing of measurement transects is too wide and point density too low, important physiographical features may be missed, causing errors in the resulting bathymetric-contour maps and storage calculations. Wilson and Richards (2006) show that lake capacity computed from bathymetric surveys decreases as transect-interval spacing increases. Storage capacity calculated from 400- and 800-ft transect intervals differed by 15.6 and 36.8 percent, respectively, from storage capacity calculated using 50-ft transect intervals.

The effect of water-depth measurement density on reservoir capacity and firm-yield estimation was examined using three reservoirs which represent typical reservoir sizes and shapes in Massachusetts. Upper Sackett Reservoir, located in Pittsfield, Mass., is the smallest of the three reservoirs, with an area of 19 acres and a gently sloping "U"-shaped lake-bottom topography. Cohasse Reservoir in Southbridge, Mass., is a long, narrow valley impoundment with a surface area of 


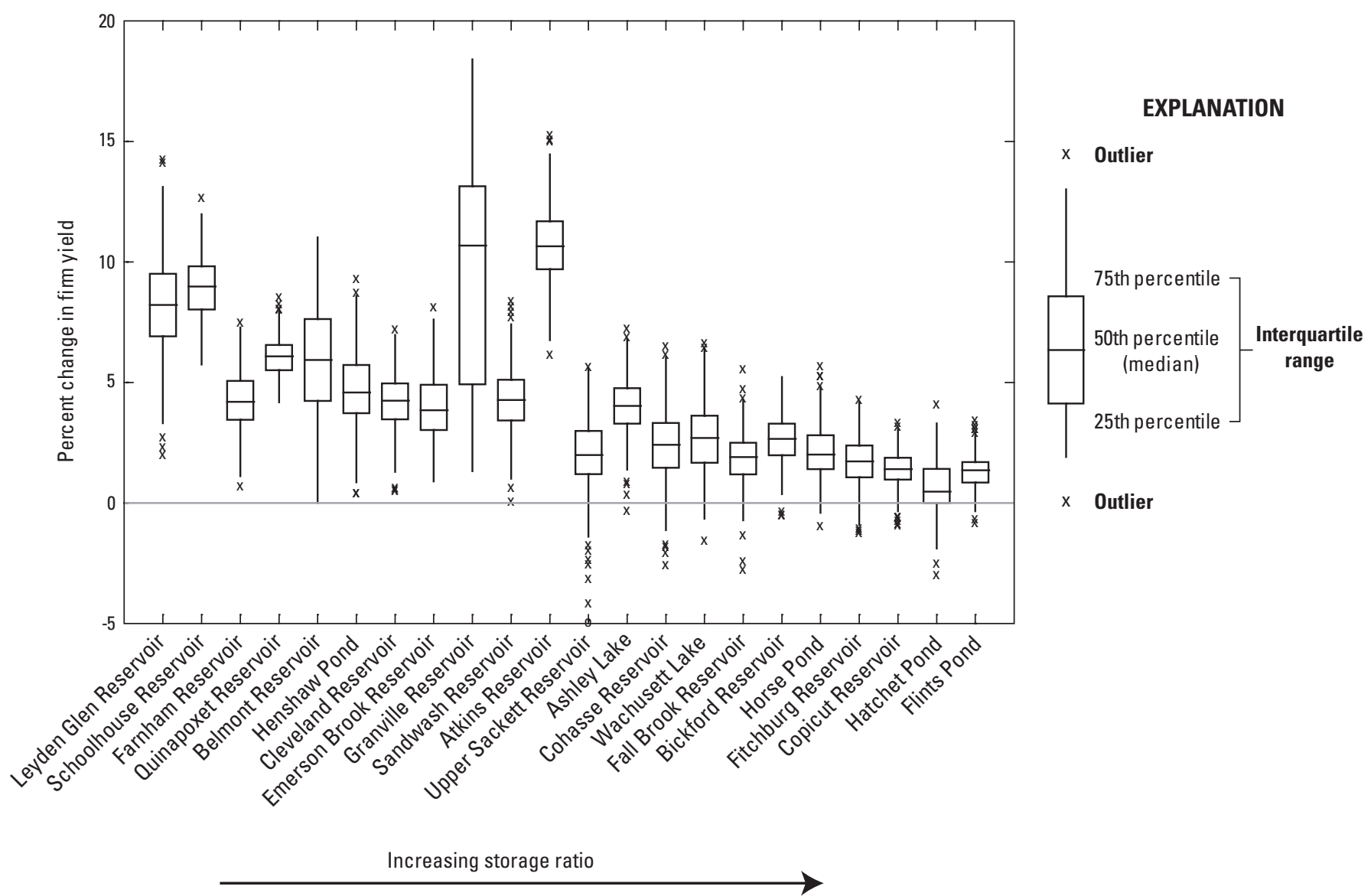

Figure 5. Percent change in firm yield of selected reservoirs in Massachusetts after accounting for potential errors in daily streamflow in 500 Monte Carlo simulations. For the purpose of this analysis, all reservoirs were simulated as single-reservoir systems. Marlborough, Leominster, Wakefield, and Worcester have water-supply connections with the Massachusetts Water Resources Authority in addition to reservoirs listed here.

56 acres and a steeply sloping "V"-shaped lake bottom. South Reservoir in Winchester, Mass., is the largest of the three reservoirs with an area of 72 acres and a relatively flat lake bottom. The area-to-perimeter ratios of Cohasse Reservoir, South Reservoir, and Upper Sackett Reservoir were 154.9, 183.0, and 202.6, respectively. Lake depths ranged from 45 to $53 \mathrm{ft}$, and surveys from all three reservoirs included high water-depth measurement densities. Original transect spacing for these three lakes ranged from 15 to 50 meters (m) and always included at least one complete shoreline-perimeter transect (table 6).

Survey measurements were interpolated using the TOPOGRID command in ARC/INFO (Environmental Systems Research Institute, Inc., 1994) to create bathymetric surfaces for each reservoir. Reservoir storage capacity was computed using the Volume tool in the 3D Analyst toolbox (Environmental Systems Research Institute, Inc., 1994). For the purposes of this uncertainty analysis, this bathymetric surface was assumed to be the true lake bottom from which water depths at points along hypothetical transects of varying spacings and patterns using a GIS were measured. Hypothetical transect lines were applied in three patterns: parallel lines across the width of the lake, perpendicular gridded lines across the width and length of the lake, and gridded lines with an additional perimeter transect (fig. 6). Each transect pattern was applied using five different transect spacings: 15, 30, 60, 90, and $120 \mathrm{~m}$, respectively. Points were placed every $5 \mathrm{~m}$ along each transect and overlaid on top of the reference bathymetric surface from which the depth was obtained. Water depths along the hypothetical transects were used to create a new bathymetric surface using the same method as for the original transect measurements, in addition to calculating reservoir storage capacity and stage-storage relations. The reservoir capacity and stage-storage data were entered into the FYE, and the firm yield was calculated for each transect pattern. The storage volume and firm yield estimated from the different transect patterns were then 


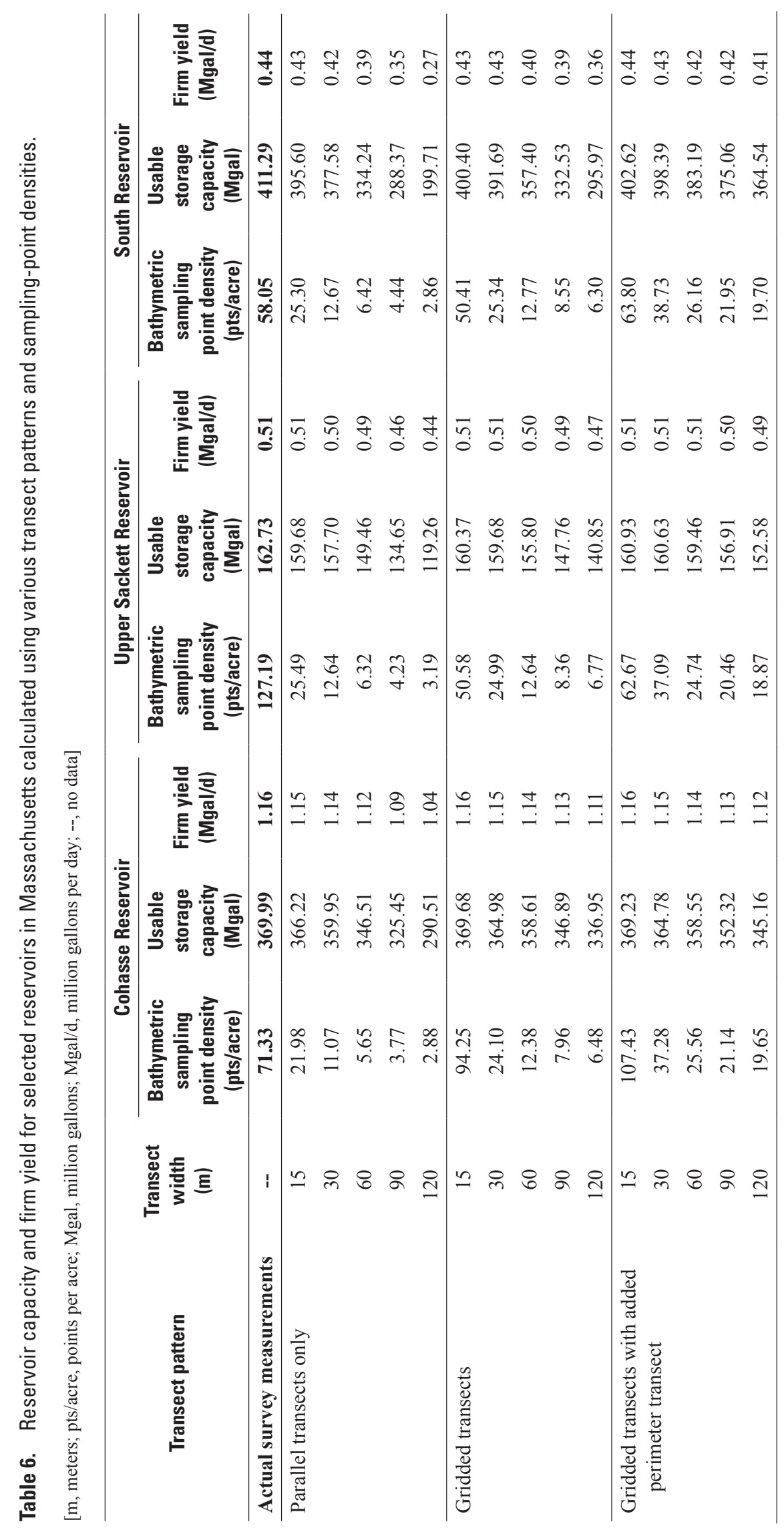




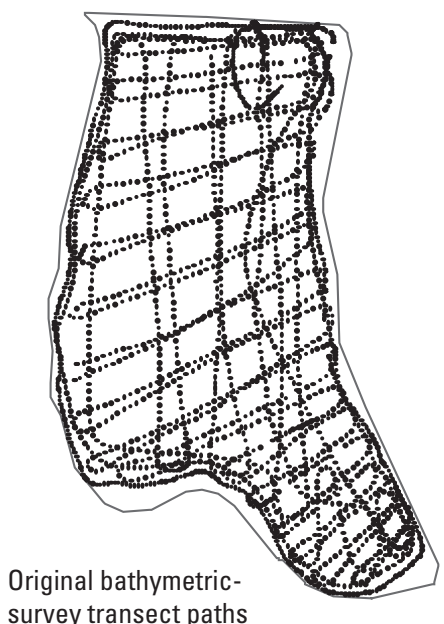

survey transect paths

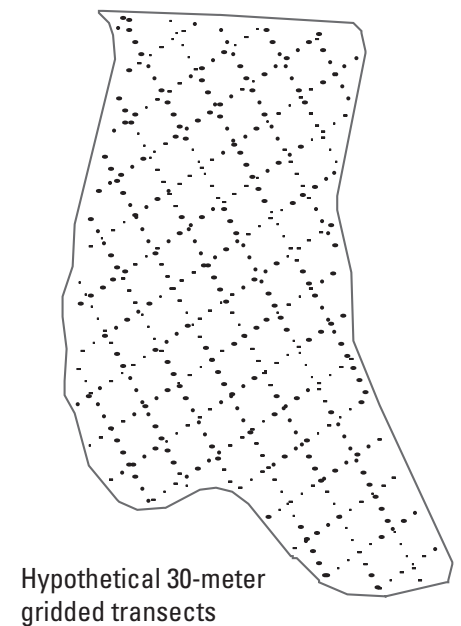

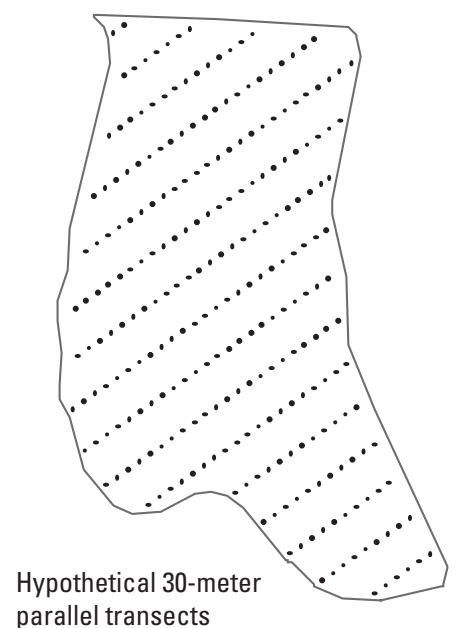

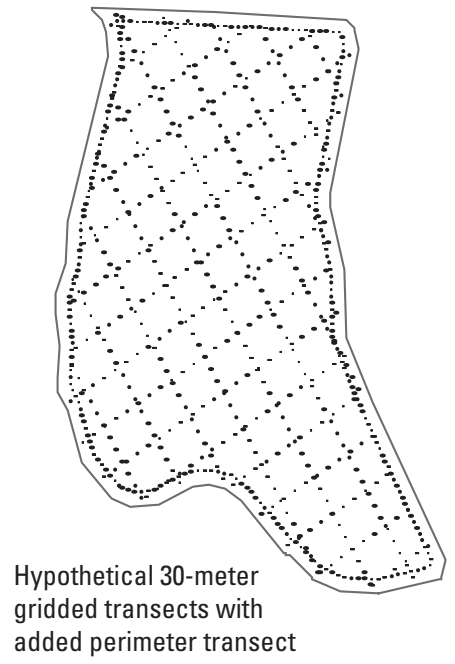

Figure 6. Original and hypothetical 30-meter transect spacing for Upper Sackett Reservoir in Pittsfield, Massachusetts. compared to the volume and firm yield estimated from original transect measurements (table 6).

Errors in estimated reservoir volume and firm yield were smallest for Cohasse Reservoir, which is long and narrow, and were largest in South Reservoir, which has a sprawling, flatbottomed lake form, indicating that reservoir shape may play a role in bathymetric accuracy. In general, parallel transects spaced farther than $15 \mathrm{~m}$ apart did a poor job of characterizing the bathymetry. Gridded transects performed better: in Cohasse and Upper Sackett Reservoirs, gridded transects spaced 60-m apart or less produced results with minimal (less than 5 percent) error rates in volume and firm yield. Gridded transects spaced $30 \mathrm{~m}$ or less apart in South Reservoir also produced minimal error rates. Adding a perimeter transect to gridded transects did not result in an appreciable improvement in volume or firm-yield accuracy in Cohasse Reservoir; however, in Upper Sackett and South Reservoirs, which have rounder reservoir shapes and lower perimeter-to-area ratios, the addition of a perimeter transect improved the volume and firm-yield estimates by roughly 50 percent. The percent changes in reservoir storage capacity and firm yield for selected reservoirs are shown in figure 7.

For each hypothetical transect-pattern scenario, the average spatial density of measurement points was calculated as the number of measurement points divided by the reservoir surface area. Measurement densities fewer than 10 measurements per acre resulted in unreliable bathymetric maps with volume errors between 6 and 51 percent and yield-estimate errors between 3 and 40 percent. Errors in volume and firmyield estimates were less than 5 percent when point densities were above more than 20 points per acre.

It is important to note that errors in bathymetric maps and calculations can also arise as a result of the methods employed during GIS processing. The type of interpolation method used, grid-cell size, and degree of smoothing can all affect the resulting bathymetric-map calculations and resulting firm yield. Bathymetry calculations may be more or less sensitive to the density of depth measurements when analyzed using different GIS techniques than those used here. 

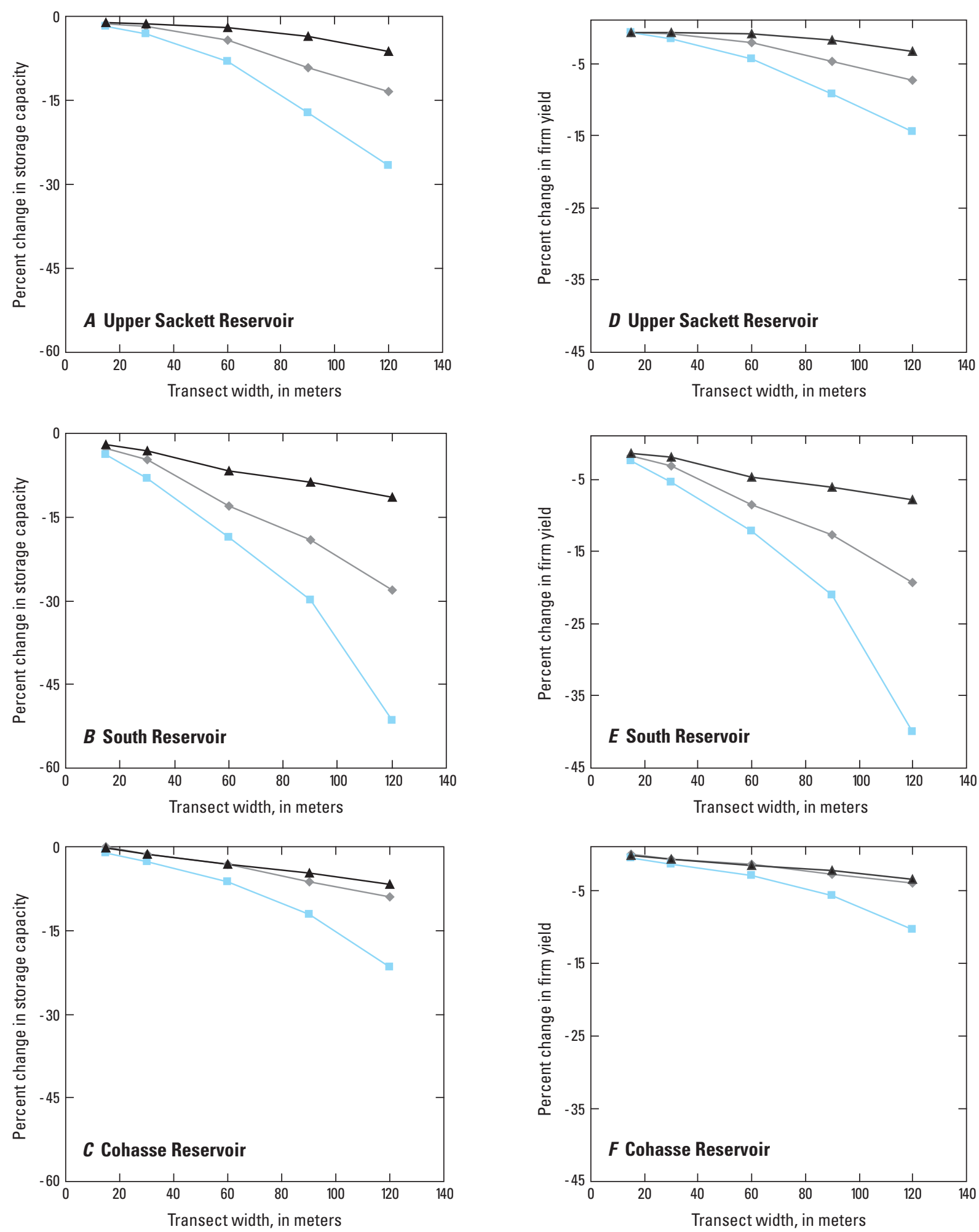

\section{EXPLANATION}

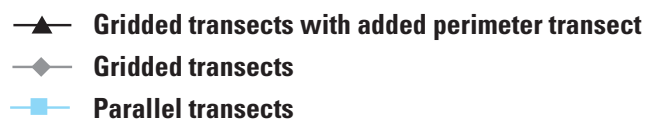

Figure 7. $A-C$, Percent change in reservoir storage capacity and $D-F$, firm yield, resulting from transect spacings and patterns for three study reservoirs in Massachusetts. 


\section{Validation of Groundwater Parameters}

Reservoir-aquifer geometry in the FYE is parameterized by (1) the length of shoreline in contact with sand and gravel, (2) the distance from the reservoir shoreline to the aquifer boundary, and (3) the transmissivity of the aquifer (Archfield and Carlson, 2006). The first two of these parameters are estimated by intersecting reservoir-shoreline locations with maps of sand and gravel deposits (Office of Geographic and Environmental Information, 2004). The calculated perimeter length is highly sensitive to the spatial scale of the lakeboundary map layer, and little guidance is available regarding proper map scale for estimating this parameter. Transmissivity values are based on published USGS hydrologic atlases, which lack numerical and geographical precision and are unavailable for some areas of Massachusetts.

Archfield and Carlson (2006) show that the FYE model is most sensitive to the transmissivity of the aquifer and length of shoreline in contact with sand and gravel. Groundwater flows into and out of the reservoir are highest during periods when reservoir stage is changing, so inaccuracies in these calculations due to parameter estimation will be most pronounced during periods of extreme reservoir storage changes. In the case where either of these two groundwater parameters is overestimated, the FYE will overestimate groundwater flows entering the reservoir during drought periods. This would cause the simulated reservoir stages to be too high and could lead to an overestimation of firm yield.

An attempt was made to use observational data to calibrate estimates of groundwater parameters. The FYE model was modified to accept observed water-use withdrawal rates in place of the yield in the water-balance equation and also to output daily reservoir stage. Simulated reservoir stage was then compared to observed reservoir stage. If other modelinput time series such as streamflow, precipitation, and wateruse withdrawal volumes, are known with certainty, groundwater parameters can be calibrated by minimizing discrepancies between observed and simulated reservoir stage. The requisite observational data for a rigorous validation of groundwater parameters were not available for any of the reservoirs in the study; however a limited amount of observational data was available from water suppliers for Nagog Pond in Acton, Mass., and Atkins Reservoir in Amherst, Mass.

Observational data for Atkins Reservoir include daily precipitation, daily withdrawal volumes, and daily reservoir stage for March 1994 to September 2004. The FYE was run for this time period using observational precipitation and water-use rates. The simulation period includes several reservoir critical periods in which the reservoir stage decreases from full pool to a local minimum, then fully refills. The most severe drawdown period occurred on October 12, 2002, when the reservoir dropped to roughly 44 percent of its total capacity. Comparison of observed and simulated reservoir stages shows that reservoir stage was overestimated during these severe critical periods (fig. 8). Other deviations in the shape of the simulated stage when compared to the observed stage, such as the delayed reservoir recovery in several of the drawdown periods, are most likely caused by errors in daily streamflow, precipitation, or evaporation input time series. The consistent bias in estimating the peak drawdown stage, however, may be caused by inaccuracies in groundwater parameters.

Because the firm yield is determined from the lowest reservoir stage during a severe drawdown period, it is important that the model can accurately predict the magnitude of a drawdown. Errors in simulated reservoir stage at nearly full-pool volumes or in the timing of the simulated drawdown period or recovery period are less important in estimating the firm-yield value. Transmissivity and parameter $L$ were adjusted in order to minimize bias in the simulation during the major drawdown periods. Agreement between observed and simulated reservoir stages during the major drawdown periods improved by decreasing transmissivity and parameter $L$ by 30 to 50 percent of their original values (fig. 8). However, errors in the shape of the simulated daily reservoir stage remain due to errors in the streamflow, precipitation, and evaporation time series. Because of errors in the other input data series, the values for transmissivity and parameter $L$ could not be calibrated with a high degree of precision, but the results of the simulations indicate that initial estimates of transmissivity and perimeter length in contact with the aquifer may have been too high. Despite a lack of precision in these variables, the inclusion of groundwater contributions did improve the agreement between observed and simulated reservoir stages compared to a simulation using only streamflow and climate inputs to the system.

Atkins Reservoir is almost fully surrounded by sand and gravel deposits; however, surficial-geology maps show that only a small portion of the area of Nagog Pond intersects with sand and gravel deposits. In addition, estimated transmissivity values for this area are low, indicating a relatively limited groundwater influence in this reservoir. Available data for Nagog Pond include once-monthly reservoir stage observations and total monthly water-use withdrawal volumes for the period of February 1975 to September 1984. Monthly withdrawal data were disaggregated into daily withdrawals by dividing the monthly total by the number of days in each month. These daily values were used in place of the yield term in the modified FYE model, and simulated stages were compared to observed reservoir stages. Drawdown periods are less severe in the observed record for Nagog Pond than for Atkins Reservoir. The lowest reservoir stage occurred in November 1980, when reservoir storage was at 62 percent of maximum capacity.

Comparison of the simulated reservoir stage with the observed reservoir stage shows a slight underestimation of reservoir stage during drawdown periods (fig. 9). There is also an overall negative bias in the last half of the simulation, from about 1980 to 1984. A consistent deviation of modeled reservoir stage from measured stage, such as that seen here, is most likely caused by errors in the streamflow, precipitation, or evaporation input data and not errors in groundwater parameters. Nagog Pond has fewer nearby climate stations than other reservoirs in the study, making the meteorological 


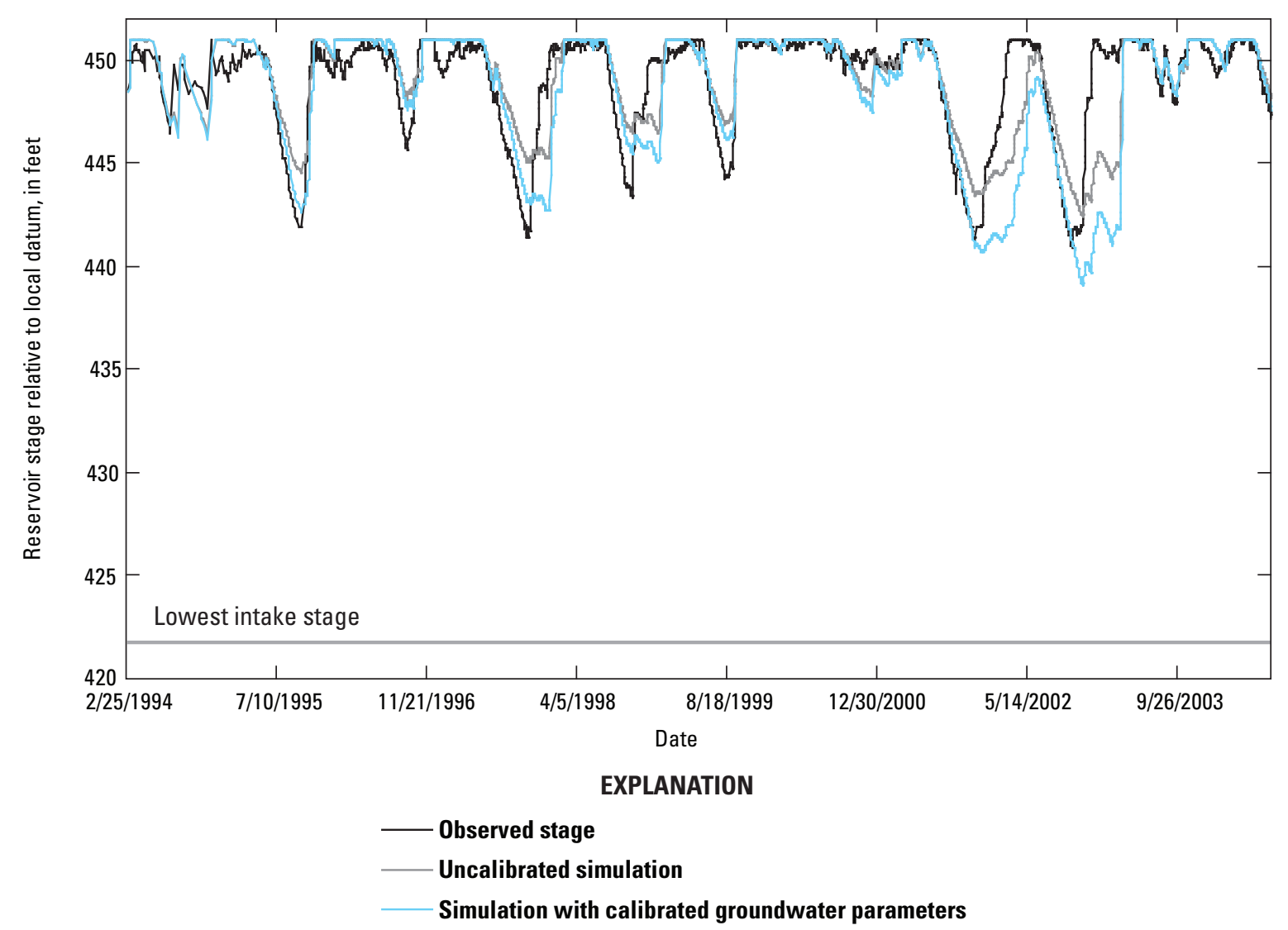

Figure 8. Daily simulated and observed reservoir stage for Atkins Reservoir in Amherst, Massachusetts.

input data more uncertain for this reservoir. Increasing the groundwater parameters for transmissivity and $L$ by as much as 300 percent resulted in only minor effects on the simulated stage of this reservoir (fig. 9); parameter adjustments greater than this are unrealistic based on geologic maps of the area. In this case, it is most likely that errors in the prediction of daily reservoir stage during droughts are due to errors in other input data, and a calibration of the groundwater parameters for this reservoir cannot be determined.

Groundwater is an important component of the water balance for many reservoirs in Massachusetts. The ability to accurately estimate groundwater flows in and out of these reservoirs is necessary for an accurate firm-yield estimate. Archfield and Carlson (2006) show that changes in the groundwater parameters $L$ and $T$ of 80 percent can lead to 10- to 40-percent changes in the firm yield.. Further study is needed in order to validate these parameters, improve methods for their estimation, and improve the numerical methods with which they are incorporated in the model. In particular, accurate time series for observed streamflow and precipitation are needed so that comparisons of observed and simulated reservoir stages are not confounded by errors in the estimation of these inputs.

\section{Effect of Drought Severity on Firm- Yield Estimates}

A system's firm yield depends largely on the severity of the drought used in its estimation. The FYE calculates firm yield by increasing reservoir yield in the water-balance equation throughout the simulation period, until the reservoir fails. A firm yield calculated using a very severe or extended drought will be lower than a yield calculated using a milder, shorter drought. For most reservoirs in Massachusetts, the multiple-year drought of the mid-1960s was the most severe drought on record; however, other severe droughts occurred 


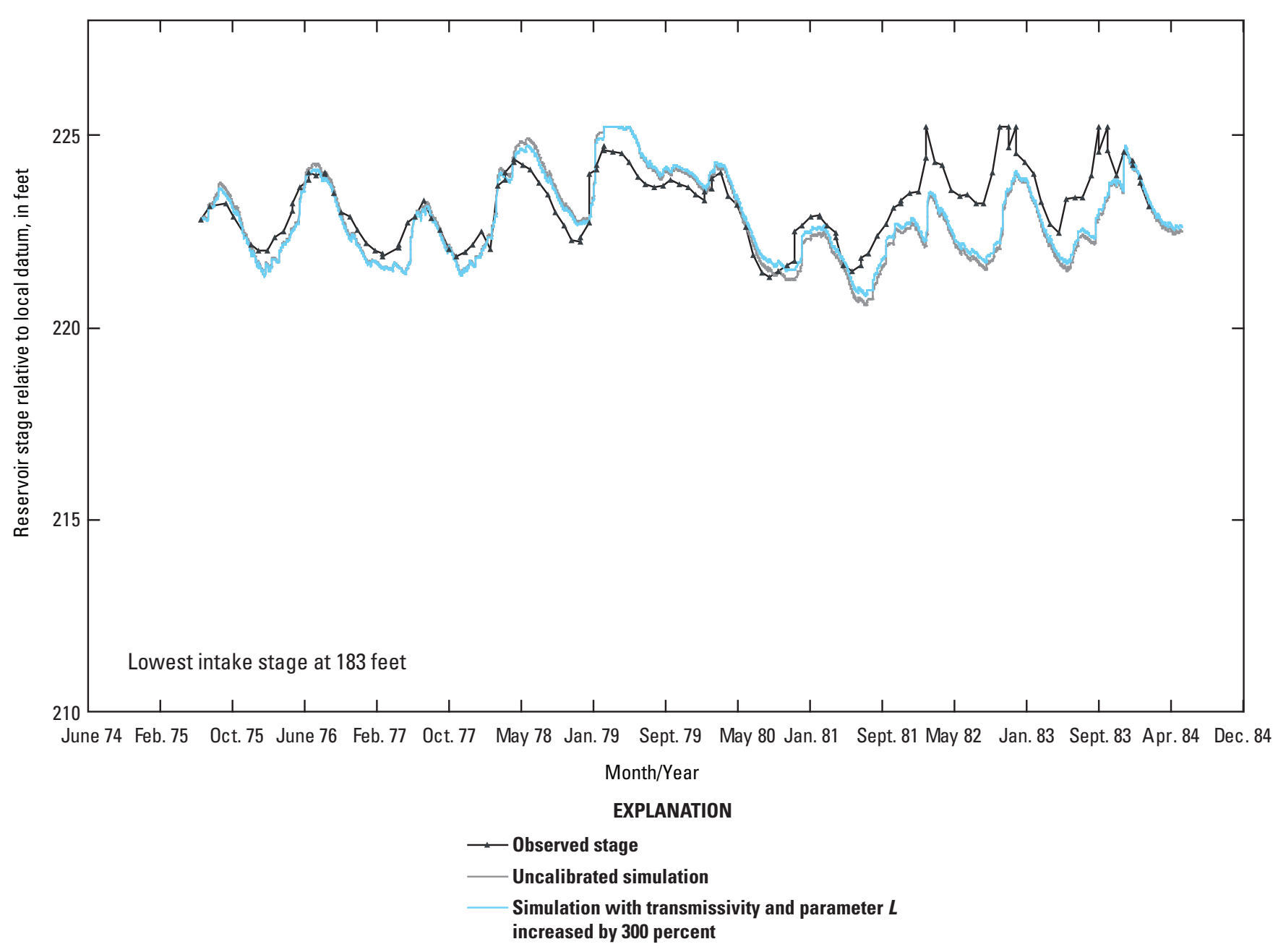

Figure 9. Daily simulated and observed reservoir stage for Nagog Pond in Concord, Massachusetts.

during the 1980s and in 2002. Differences in the firm yields determined from the droughts in the historical record and the behavior of reservoirs under different drought conditions have not been evaluated in Massachusetts.

Drought periods can be defined in terms of either a prolonged period of below-average precipitation (meteorological drought) or streamflow (hydrologic drought). Hydrologicdrought periods may differ slightly from meteorologicaldrought periods because of evaporation and groundwater storage. Because the firm yield is more sensitive to streamflow than to direct precipitation, hydrologic drought is considered in this analysis.

Hydrologic droughts can be characterized in terms of their duration and intensity. The 3-month moving streamflow average was calculated from the daily streamflow record and compared to the average seasonal streamflow from the entire long-term streamflow record. Seasonal flow periods were defined as: spring (March through May), summer (June through August), autumn (September through November), and winter (December through February). The drought duration was defined as the number of consecutive months for which the 3-month moving average is less than the average seasonal flow. Drought intensity was calculated as the percent difference between the magnitude of the 3-month moving average streamflow and the average seasonal streamflow over the duration of the drought, expressed as a percentage of average streamflow.

Using the daily streamflow record generated from the SYE, hydrologic droughts were characterized for reservoirs in Massachusetts. Analysis of droughts focused on the droughts in the 1960s, 1980s, and 2002, as these were the three most severe droughts for most Massachusetts reservoirs. The drought of the 1960s is notable for its extended length. Durations for this drought ranged from 20 to 69 months, with a median of 38 months (table 7, fig. 10). Average 3-month streamflows during this drought were 48 to 74 percent below average over the duration of the 1960s drought. Droughts in the 1980s and 2002 were shorter in duration but in some cases 
Table 7. Firm yields and reliability of reservoirs in Massachusetts calculated using three major droughts in the historical record. For the Worcester have water-supply connections with the Massachusetts Water Resources Authority in addition to reservoirs listed here.

[Dates are in months and years from 1964 to 2002; Mgal/d, million gallons per day]

\begin{tabular}{|c|c|c|c|c|c|c|c|c|c|c|}
\hline & \multicolumn{5}{|c|}{ 1960s drought } & \multicolumn{5}{|c|}{ 1980s drought } \\
\hline & $\begin{array}{l}\text { Stor- } \\
\text { age } \\
\text { ratio }\end{array}$ & $\begin{array}{l}\text { Drought } \\
\text { length } \\
\text { (months) }\end{array}$ & $\begin{array}{c}\text { Drought } \\
\text { intensity } \\
\text { (percent be- } \\
\text { low average } \\
\text { streamflow) }\end{array}$ & $\begin{array}{c}\text { Firm } \\
\text { yield } \\
\text { (Mgal/d) }\end{array}$ & $\begin{array}{c}\text { Date of } \\
\text { maximum } \\
\text { storage } \\
\text { depletion }\end{array}$ & $\begin{array}{l}\text { Drought } \\
\text { length } \\
\text { (months) }\end{array}$ & $\begin{array}{c}\text { Drought } \\
\text { intensity } \\
\text { (percent be- } \\
\text { low average } \\
\text { streamflow) }\end{array}$ & $\begin{array}{c}\text { Firm } \\
\text { yield } \\
\text { (Mgal/d) }\end{array}$ & $\begin{array}{c}\text { Date of } \\
\text { maximum } \\
\text { storage } \\
\text { depletion }\end{array}$ & $\begin{array}{c}\text { Reli- } \\
\text { ability } \\
\text { (per- } \\
\text { cent) }\end{array}$ \\
\hline Accord Pond & 0.60 & 20 & 47.9 & 0.67 & Sep-66 & 22 & 54.8 & 0.66 & Sep-81 & 100.0 \\
\hline Amythest Brook Intake & 0.01 & 36 & 68.2 & 0.06 & Nov-64 & 5 & 58.6 & 0.10 & Sep-83 & 98.7 \\
\hline Ashley Lake & 0.37 & 36 & 68.1 & 1.37 & Feb-66 & 24 & 35.8 & 1.91 & Feb-81 & 98.4 \\
\hline Atkins Reservoir & 0.31 & 36 & 66.9 & 1.16 & Oct-66 & 20 & 39.2 & 1.69 & Oct-81 & 96.4 \\
\hline Bearhole Reservoir & 0.04 & 47 & 58.8 & 1.40 & Nov-64 & 8 & 72.8 & 1.84 & Feb-81 & 98.4 \\
\hline Belmont Reservoir & 0.17 & 36 & 73.6 & 0.12 & Feb-66 & 23 & 39.1 & 0.16 & Feb-81 & 98.7 \\
\hline Bickford Reservoir & 0.59 & 36 & 66.2 & 1.91 & Jan-66 & 17 & 41.6 & 2.73 & Jan-81 & 98.4 \\
\hline Borden Brook Reservoir & 0.70 & 37 & 57.8 & 6.34 & Nov-66 & 11 & 39.0 & 8.88 & Nov-81 & 96.6 \\
\hline Cleveland Reservoir & 0.26 & 64 & 53.6 & 9.18 & Feb-65 & 8 & 80.2 & 9.39 & Feb-81 & 99.9 \\
\hline Cohasse Reservoir & 0.40 & 69 & 57.2 & 1.16 & Feb-66 & 12 & 37.4 & 1.73 & Nov-85 & 97.0 \\
\hline Crystal Lake & 0.49 & 47 & 65.5 & 0.40 & Nov-66 & 11 & 59.3 & 0.68 & Sep-85 & 97.2 \\
\hline Distributing Reservoir & 0.01 & 36 & 67.2 & 0.15 & Nov-64 & 5 & 54.8 & 0.23 & Sep-83 & 98.1 \\
\hline Doane Pond & 0.11 & 47 & 69.5 & 0.21 & Feb-66 & 8 & 61.0 & 0.34 & Nov-80 & 95.7 \\
\hline Echo Lake & 0.79 & 38 & 66.7 & 0.83 & Jan-67 & 11 & 46.4 & 1.33 & Nov-85 & 97.3 \\
\hline Emerson Brook & 0.26 & 38 & 62.3 & 1.22 & Feb-66 & 15 & 59.1 & 1.59 & Nov-85 & 98.5 \\
\hline Fall Brook Reservoir & 0.57 & 36 & 68.2 & 0.84 & Feb-66 & 17 & 43.0 & 1.31 & Oct-81 & 97.1 \\
\hline Fitchburg Reservoir & 0.70 & 48 & 69.3 & 1.22 & Mar-67 & 15 & 61.7 & 1.97 & Jan-86 & 96.7 \\
\hline Goodfellow Pond & 0.04 & 36 & 67.4 & 0.07 & Nov-64 & 17 & 41.9 & 0.10 & Nov-80 & 99.2 \\
\hline Granville Reservoir & 0.26 & 38 & 57.9 & 3.03 & Feb-66 & 12 & 61.9 & 4.21 & Feb-81 & 98.2 \\
\hline Hatchet Brook Reservoir \#3 & 0.31 & 69 & 59.5 & 0.32 & Feb-66 & 11 & 36.5 & 0.49 & Feb-85 & 97.1 \\
\hline Hatchet Brook Reservoir \#4 & 0.87 & 69 & 58.8 & 0.51 & Dec-66 & 12 & 39.1 & 0.81 & Nov-86 & 95.2 \\
\hline Hatchet Brook Reservoir \#5 & 0.37 & 69 & 58.2 & 0.57 & Feb-66 & 12 & 38.9 & 0.86 & Nov-85 & 97.1 \\
\hline Hatchet Pond & 1.55 & 69 & 63.2 & 0.21 & Jan-67 & 14 & 33.5 & 0.35 & Feb-89 & 94.1 \\
\hline Hawley Reservoir & 0.01 & 36 & 67.1 & 0.17 & Nov-64 & 21 & 40.2 & 0.31 & Sep-80 & 98.4 \\
\hline Haynes Reservoir & 0.69 & 36 & 70.6 & 0.26 & Oct-66 & 23 & 29.8 & 0.44 & Mar-89 & 96.6 \\
\hline Henshaw Pond & 0.21 & 47 & 68.3 & 0.35 & Feb-66 & 8 & 59.6 & 0.57 & Feb-81 & 95.9 \\
\hline Hill Reservoir & 0.01 & 36 & 64.9 & 0.65 & Dec-64 & 18 & 42.5 & 0.97 & Feb-81 & 98.4 \\
\hline Holden Reservoir \#1 & 0.37 & 47 & 64.1 & 2.58 & Nov-66 & 16 & 38.4 & 4.20 & Nov-88 & 95.5 \\
\hline Holden Reservoir \#2 & 0.70 & 47 & 68.9 & 0.47 & Mar-67 & 16 & 44.2 & 0.78 & Mar-89 & 96.2 \\
\hline Horse Pond & 0.62 & 47 & 69.0 & 0.50 & Jan-67 & 16 & 43.4 & 0.87 & Mar-89 & 95.7 \\
\hline Kendall Reservoir & 0.91 & 36 & 64.9 & 1.49 & Nov-66 & 23 & 25.3 & 2.16 & Nov-88 & 95.9 \\
\hline Kettle Brook Reservoir \#1 & 0.03 & 47 & 68.4 & 0.12 & Nov-64 & 12 & 48.2 & 0.22 & Nov-84 & 96.6 \\
\hline Kettle Brook Reservoir \#2 & 0.50 & 47 & 71.0 & 0.27 & Jan-67 & 16 & 46.3 & 0.47 & Mar-89 & 95.9 \\
\hline Kettle Brook Reservoir \#3 & 0.42 & 47 & 69.6 & 0.38 & Jan-67 & 16 & 44.3 & 0.65 & Jan-89 & 96.0 \\
\hline Kettle Brook Reservoir \#4 & 0.56 & 47 & 67.2 & 1.08 & Jan-67 & 16 & 41.3 & 1.83 & Jan-89 & 96.0 \\
\hline Leyden Glen Reservoir & 0.02 & 58 & 57.1 & 0.69 & Feb-65 & 14 & 11.7 & 0.71 & Feb-81 & 99.9 \\
\hline Lovell Reservoir & 0.23 & 47 & 71.7 & 1.16 & Feb-66 & 15 & 61.3 & 1.80 & Nov-85 & 98.0 \\
\hline Lynde Brook Reservoir & 0.35 & 47 & 66.6 & 1.51 & Feb-66 & 8 & 58.4 & 2.59 & Feb-81 & 95.9 \\
\hline Main Reservoir & 0.09 & 35 & 64.2 & 0.63 & Dec-65 & 23 & 59.1 & 0.77 & Oct-80 & 99.4 \\
\hline
\end{tabular}


purpose of this analysis, all reservoirs were simulated as single-reservoir systems. Marlborough, Leominster, Wakefield, and

\begin{tabular}{|c|c|c|c|c|c|c|c|c|c|c|}
\hline \multicolumn{3}{|c|}{ 1980s drought } & \multicolumn{8}{|c|}{2002 drought } \\
\hline $\begin{array}{l}\text { Number } \\
\text { of failure } \\
\text { events }\end{array}$ & $\begin{array}{l}\text { Average } \\
\text { failure } \\
\text { duration } \\
\text { (days) }\end{array}$ & $\begin{array}{c}\text { Average } \\
\text { yield deficit } \\
\text { during } \\
\text { failure } \\
\text { (Mgal/d) }\end{array}$ & $\begin{array}{l}\text { Drought } \\
\text { length } \\
\text { (months) }\end{array}$ & $\begin{array}{c}\text { Drought } \\
\text { intensity } \\
\text { (percent be- } \\
\text { low average } \\
\text { streamflow) }\end{array}$ & $\begin{array}{c}\text { Firm yield } \\
\text { (Mgal/d) }\end{array}$ & $\begin{array}{c}\text { Date of } \\
\text { maximum } \\
\text { storage } \\
\text { depletion }\end{array}$ & $\begin{array}{c}\text { Reli- } \\
\text { ability } \\
\text { (per- } \\
\text { cent) }\end{array}$ & $\begin{array}{c}\text { Number } \\
\text { of failure } \\
\text { events }\end{array}$ & $\begin{array}{l}\text { Average } \\
\text { failure } \\
\text { duration } \\
\text { (days) }\end{array}$ & $\begin{array}{c}\text { Average } \\
\text { yield deficit } \\
\text { during } \\
\text { failure } \\
\text { (Mgal/d) }\end{array}$ \\
\hline 0 & 0 & 0.0 & 13 & 49.8 & 0.75 & Oct- 02 & 99.2 & 14 & 10 & 9.2 \\
\hline 17 & 12 & 0.6 & 22 & 55.3 & 0.10 & Jan-02 & 98.8 & 13 & 15 & 0.8 \\
\hline 13 & 20 & 35.2 & 23 & 54.7 & 1.81 & Feb-03 & 99.0 & 4 & 39 & 74.8 \\
\hline 20 & 29 & 36.5 & 22 & 54.9 & 1.49 & Nov-02 & 98.0 & 11 & 29 & 32.7 \\
\hline 19 & 14 & 14.4 & 17 & 54.2 & 1.81 & Oct-02 & 98.6 & 14 & 16 & 16.5 \\
\hline 8 & 27 & 3.3 & 25 & 55.7 & 0.16 & Mar-02 & 98.7 & 7 & 30 & 3.7 \\
\hline 13 & 20 & 69.3 & 20 & 49.2 & 2.49 & Nov-02 & 98.9 & 10 & 17 & 49.0 \\
\hline 16 & 34 & 246.3 & 12 & 47.0 & 8.70 & Nov-02 & 97.0 & 15 & 33 & 228.9 \\
\hline 2 & 6 & 30.3 & 10 & 34.8 & 12.87 & Jan-02 & 94.3 & 51 & 18 & 155.2 \\
\hline 15 & 32 & 52.3 & 10 & 40.5 & 1.45 & Dec-02 & 99.0 & 4 & 41 & 56.3 \\
\hline 16 & 28 & 17.6 & 10 & 58.3 & 0.65 & Nov-02 & 97.6 & 12 & 32 & 19.6 \\
\hline 14 & 22 & 2.8 & 20 & 49.5 & 0.21 & Oct-02 & 98.7 & 12 & 17 & 1.9 \\
\hline 30 & 23 & 4.9 & 20 & 56.7 & 0.27 & Jan-02 & 98.9 & 7 & 26 & 4.1 \\
\hline 11 & 40 & 58.8 & 20 & 54.6 & 1.17 & Nov-02 & 98.2 & 9 & 32 & 37.2 \\
\hline 7 & 35 & 40.1 & 12 & 57.5 & 1.72 & Feb-02 & 97.7 & 12 & 31 & 37.7 \\
\hline 13 & 36 & 40.9 & 20 & 50.5 & 1.13 & Nov-02 & 98.3 & 13 & 21 & 19.3 \\
\hline 9 & 58 & 95.7 & 22 & 53.7 & 1.94 & Dec-02 & 97.0 & 5 & 96 & 160.3 \\
\hline 13 & 10 & 0.8 & 20 & 50.3 & 0.10 & Oct- 02 & 99.2 & 13 & 10 & 0.8 \\
\hline 11 & 26 & 78.4 & 12 & 47.1 & 4.53 & Nov-02 & 97.7 & 13 & 28 & 95.7 \\
\hline 18 & 26 & 11.2 & 10 & 56.9 & 0.40 & Mar-02 & 99.1 & 4 & 35 & 12.0 \\
\hline 29 & 26 & 23.2 & 10 & 41.9 & 0.71 & Dec-02 & 97.0 & 21 & 23 & 17.3 \\
\hline 16 & 29 & 23.7 & 10 & 41.5 & 0.72 & Dec-02 & 99.0 & 4 & 41 & 28.2 \\
\hline 23 & 41 & 14.9 & 10 & 59.6 & 0.31 & Feb-03 & 96.4 & 11 & 52 & 17.3 \\
\hline 22 & 12 & 1.8 & 22 & 54.6 & 0.28 & Feb-02 & 98.8 & 16 & 12 & 1.6 \\
\hline 19 & 29 & 9.2 & 20 & 52.9 & 0.38 & Nov-02 & 98.1 & 14 & 22 & 5.9 \\
\hline 32 & 21 & 7.9 & 20 & 55.3 & 0.49 & Feb-02 & 98.2 & 11 & 27 & 9.2 \\
\hline 22 & 12 & 5.2 & 22 & 52.2 & 0.91 & Feb-02 & 98.8 & 17 & 12 & 4.5 \\
\hline 18 & 40 & 125.4 & 20 & 51.4 & 3.48 & Nov-02 & 97.9 & 7 & 49 & 131.3 \\
\hline 10 & 61 & 42.5 & 20 & 56.1 & 0.69 & Dec-02 & 97.4 & 6 & 70 & 43.6 \\
\hline 23 & 30 & 22.5 & 20 & 56.1 & 0.74 & Nov-02 & 97.5 & 16 & 25 & 16.1 \\
\hline 19 & 35 & 71.2 & 20 & 48.5 & 1.86 & Nov-02 & 98.6 & 9 & 25 & 41.0 \\
\hline 21 & 26 & 4.2 & 20 & 55.5 & 0.15 & Dec-01 & 99.3 & 7 & 17 & 1.8 \\
\hline 10 & 65 & 26.4 & 20 & 58.1 & 0.39 & Dec-02 & 97.8 & 3 & 119 & 39.9 \\
\hline 12 & 54 & 29.7 & 20 & 56.7 & 0.53 & Dec-02 & 97.9 & 3 & 114 & 51.0 \\
\hline 9 & 72 & 114.2 & 20 & 54.4 & 1.54 & Dec-02 & 97.8 & 3 & 1,173 & 152.4 \\
\hline 1 & 3 & 2.6 & 10 & 40.8 & 1.19 & Feb-02 & 98.5 & 20 & 12 & 16.0 \\
\hline 7 & 47 & 64.2 & 12 & 60.3 & 1.82 & Mar-02 & 97.8 & 8 & 44 & 61.1 \\
\hline 16 & 42 & 85.3 & 20 & 53.8 & 2.14 & Dec-02 & 97.9 & 6 & 56 & 95.9 \\
\hline 5 & 18.4 & 7.4 & 4 & 57.6 & 1.06 & Oct-02 & 95.4 & 33 & 22.2 & 18.6 \\
\hline
\end{tabular}


Table 7. Firm yields and reliability of reservoirs in Massachusetts calculated using three major droughts in the historical record. For the Worcester have water-supply connections with the Massachusetts Water Resources Authority in addition to reservoirs listed here.-Continued

[Dates are in months and years from 1964 to 2002; Mgal/d, million gallons per day]

\begin{tabular}{|c|c|c|c|c|c|c|c|c|c|c|}
\hline & \multicolumn{5}{|c|}{ 1960s drought } & \multicolumn{5}{|c|}{ 1980s drought } \\
\hline & $\begin{array}{c}\text { Stor- } \\
\text { age } \\
\text { ratio }\end{array}$ & $\begin{array}{l}\text { Drought } \\
\text { length } \\
\text { (months) }\end{array}$ & $\begin{array}{c}\text { Drought } \\
\text { intensity } \\
\text { (percent be- } \\
\text { low average } \\
\text { streamflow) }\end{array}$ & $\begin{array}{c}\text { Firm } \\
\text { yield } \\
\text { (Mgal/d) }\end{array}$ & $\begin{array}{c}\text { Date of } \\
\text { maximum } \\
\text { storage } \\
\text { depletion }\end{array}$ & $\begin{array}{l}\text { Drought } \\
\text { length } \\
\text { (months) }\end{array}$ & $\begin{array}{c}\text { Drought } \\
\text { intensity } \\
\text { (percent be- } \\
\text { low average } \\
\text { streamflow) }\end{array}$ & $\begin{array}{c}\text { Firm } \\
\text { yield } \\
\text { (Mgal/d) }\end{array}$ & $\begin{array}{c}\text { Date of } \\
\text { maximum } \\
\text { storage } \\
\text { depletion }\end{array}$ & $\begin{array}{c}\text { Reli- } \\
\text { ability } \\
\text { (per- } \\
\text { cent) }\end{array}$ \\
\hline Mare Meadow Reservoir & 1.36 & 36 & 67.4 & 2.68 & Dec-66 & 23 & 26.9 & 3.70 & Mar-89 & 97.1 \\
\hline Meetinghouse Reservoir & 0.97 & 36 & 68.3 & 1.12 & Dec-66 & 23 & 28.2 & 1.78 & Mar-89 & 95.6 \\
\hline Middle Reservoir & 1.15 & 47 & 70.0 & 0.15 & Jan-67 & 14 & 39.2 & 0.28 & Aug-89 & 96.4 \\
\hline Millham Reservoir & 0.20 & 36 & 61.8 & 1.79 & Nov-65 & 16 & 44.3 & 2.47 & Feb-81 & 97.7 \\
\hline Montgomery Reservoir & 0.18 & 38 & 59.6 & 1.13 & Feb-66 & 8 & 69.2 & 1.35 & Feb-81 & 99.0 \\
\hline Morse Reservoir & 0.29 & 36 & 70.1 & 0.17 & Feb-66 & 11 & 26.1 & 0.28 & Feb-81 & 96.4 \\
\hline North Reservoir & 0.98 & 47 & 66.9 & 0.39 & Jan-67 & 14 & 36.4 & 0.65 & Sep-89 & 96.4 \\
\hline Notown Reservoir & 0.37 & 36 & 64.9 & 2.13 & Feb-66 & 17 & 40.3 & 3.11 & Feb-81 & 97.9 \\
\hline Pine Hill Reservoir & 1.00 & 36 & 64.4 & 5.52 & Nov-66 & 23 & 24.7 & 7.86 & Mar-89 & 95.9 \\
\hline Quinapoxet Reservoir & 0.13 & 36 & 60.2 & 4.21 & Nov-64 & 16 & 42.2 & 4.88 & Nov- 80 & 99.8 \\
\hline Roaring Brook Dam & 0.09 & 59 & 57.8 & 0.98 & Dec-64 & 8 & 78.6 & 1.23 & Nov-80 & 99.4 \\
\hline Sandra Pond & 0.28 & 38 & 63.9 & 0.80 & Dec-66 & 15 & 36.4 & 1.21 & Nov-81 & 95.7 \\
\hline Sandwash Reservoir & 0.28 & 36 & 69.6 & 0.89 & Feb-66 & 24 & 36.8 & 1.31 & Feb-81 & 97.6 \\
\hline Schoolhouse Reservoir & 0.08 & 36 & 68.7 & 0.84 & Dec-64 & 22 & 36.8 & 1.11 & Feb-81 & 99.4 \\
\hline Scott Reservoir & 0.47 & 48 & 70.8 & 0.40 & Feb-66 & 15 & 63.5 & 0.61 & Nov- 85 & 97.5 \\
\hline Simonds Pond & 0.17 & 36 & 72.4 & 0.07 & Feb-65 & 11 & 28.9 & 0.08 & Feb-81 & 99.0 \\
\hline Swan Pond & 0.38 & 38 & 64.1 & 0.44 & Nov-65 & 15 & 61.1 & 0.53 & Nov-85 & 99.7 \\
\hline Upper (Leahey) Reservoir & 0.97 & 36 & 72.3 & 0.59 & Dec-66 & 23 & 36.0 & 0.88 & Mar-89 & 97.1 \\
\hline Upper Naukeag Lake & 1.43 & 46 & 73.1 & 1.72 & Mar-67 & 9 & 21.1 & 2.71 & Mar-89 & 91.7 \\
\hline Upper Sackett Reservoir & 0.35 & 36 & 71.4 & 0.50 & Sep-65 & 14 & 59.5 & 0.72 & Sep-85 & 97.7 \\
\hline Wachusett Lake & 0.55 & 36 & 66.3 & 1.08 & Oct-66 & 23 & 27.2 & 1.69 & Mar-89 & 96.6 \\
\hline Whately Reservoir & 0.02 & 59 & 60.5 & 0.09 & Nov-63 & 8 & 81.6 & 0.10 & Oct- 80 & 99.6 \\
\hline
\end{tabular}


purpose of this analysis, all reservoirs were simulated as single-reservoir systems. Marlborough, Leominster, Wakefield, and

\begin{tabular}{|c|c|c|c|c|c|c|c|c|c|c|}
\hline \multicolumn{3}{|c|}{ 1980s drought } & \multicolumn{8}{|c|}{2002 drought } \\
\hline $\begin{array}{l}\text { Number } \\
\text { of failure } \\
\text { events }\end{array}$ & $\begin{array}{l}\text { Average } \\
\text { failure } \\
\text { duration } \\
\text { (days) }\end{array}$ & $\begin{array}{l}\text { Average } \\
\text { yield deficit } \\
\text { during } \\
\text { failure } \\
\text { (Mgal/d) }\end{array}$ & $\begin{array}{l}\text { Drought } \\
\text { length } \\
\text { (months) }\end{array}$ & $\begin{array}{c}\text { Drought } \\
\text { intensity } \\
\text { (percent be- } \\
\text { low average } \\
\text { streamflow) }\end{array}$ & $\begin{array}{c}\text { Firm yield } \\
\text { (Mgal/d) }\end{array}$ & $\begin{array}{c}\text { Date of } \\
\text { maximum } \\
\text { storage } \\
\text { depletion }\end{array}$ & $\begin{array}{c}\text { Reli- } \\
\text { ability } \\
\text { (per- } \\
\text { cent) }\end{array}$ & $\begin{array}{c}\text { Number } \\
\text { of failure } \\
\text { events }\end{array}$ & $\begin{array}{l}\text { Average } \\
\text { failure } \\
\text { duration } \\
\text { (days) }\end{array}$ & $\begin{array}{l}\text { Average } \\
\text { yield deficit } \\
\text { during } \\
\text { failure } \\
\text { (Mgal/d) }\end{array}$ \\
\hline 19 & 24 & 127.6 & 20 & 50.3 & 3.21 & Dec-02 & 99.0 & 8 & 21 & 88.4 \\
\hline 31 & 22.7 & 39.7 & 20 & 51.1 & 1.52 & Nov-02 & 98.3 & 15 & 17.7 & 26.9 \\
\hline 9 & 64.6 & 15.7 & 10 & 62.4 & 0.27 & Dec-02 & 97.1 & 5 & 93.4 & 22.2 \\
\hline 17 & 21.4 & 32.3 & 10 & 54.8 & 2.43 & Nov-02 & 98.0 & 14 & 22.9 & 34.8 \\
\hline 6 & 26.8 & 23.3 & 10 & 44.9 & 1.49 & Feb-02 & 98.4 & 8 & 32.5 & 34.7 \\
\hline 24 & 23.8 & 4.6 & 20 & 52.4 & 0.24 & Nov-02 & 98.0 & 14 & 22.5 & 3.7 \\
\hline 18 & 32.6 & 19.3 & 12 & 30.3 & 0.62 & Dec-02 & 97.2 & 11 & 40.3 & 23.1 \\
\hline 13 & 26.1 & 61.6 & 20 & 47.4 & 2.95 & Nov-02 & 98.4 & 10 & 25.8 & 57.8 \\
\hline 21 & 31.4 & 226.3 & 20 & 47.3 & 6.91 & Nov-02 & 98.5 & 8 & 29.4 & 172.7 \\
\hline 3 & 13.3 & 94.8 & 19 & 45.8 & 5.04 & Nov-01 & 99.6 & 4 & 16.3 & 116.1 \\
\hline 10 & 10.5 & 8.7 & 10 & 56.9 & 1.39 & Dec-01 & 98.3 & 18 & 15.3 & 15.9 \\
\hline 12 & 57.8 & 56.4 & 20 & 53.3 & 1.04 & Dec-02 & 97.6 & 4 & 95.8 & 75.3 \\
\hline 13 & 29.2 & 26.9 & 23 & 56.1 & 1.19 & Nov-02 & 98.6 & 6 & 36.3 & 30.4 \\
\hline 10 & 9.5 & 6.0 & 23 & 54.9 & 1.12 & Feb-02 & 99.3 & 11 & 10.3 & 6.4 \\
\hline 4 & 102.0 & 51.4 & 12 & 61.9 & 0.63 & Dec-02 & 97.3 & 5 & 87.8 & 45.6 \\
\hline 9 & 17.4 & 0.9 & 20 & 54.6 & 0.09 & Feb-02 & 98.4 & 9 & 29.4 & 2.0 \\
\hline 5 & 9.2 & 20.3 & 12 & 59.1 & 0.51 & Nov-01 & 99.8 & 2 & 16.0 & 35.9 \\
\hline 11 & 42.1 & 38.7 & 25 & 53.9 & 0.78 & Dec-02 & 98.4 & 8 & 32.5 & 24.4 \\
\hline 89 & 14.9 & 33.9 & 12 & 51.2 & 2.40 & Feb-03 & 97.0 & 37 & 13.2 & 27.4 \\
\hline 11 & 34.1 & 22.4 & 24 & 54.0 & 0.63 & Oct-02 & 98.7 & 8 & 26.0 & 14.8 \\
\hline 24 & 22.9 & 35.3 & 20 & 48.9 & 1.44 & Nov-02 & 98.2 & 18 & 16.2 & 19.6 \\
\hline 5 & 13.0 & 0.9 & 10 & 59.4 & 0.15 & Nov-01 & 97.6 & 22 & 17.6 & 2.3 \\
\hline
\end{tabular}



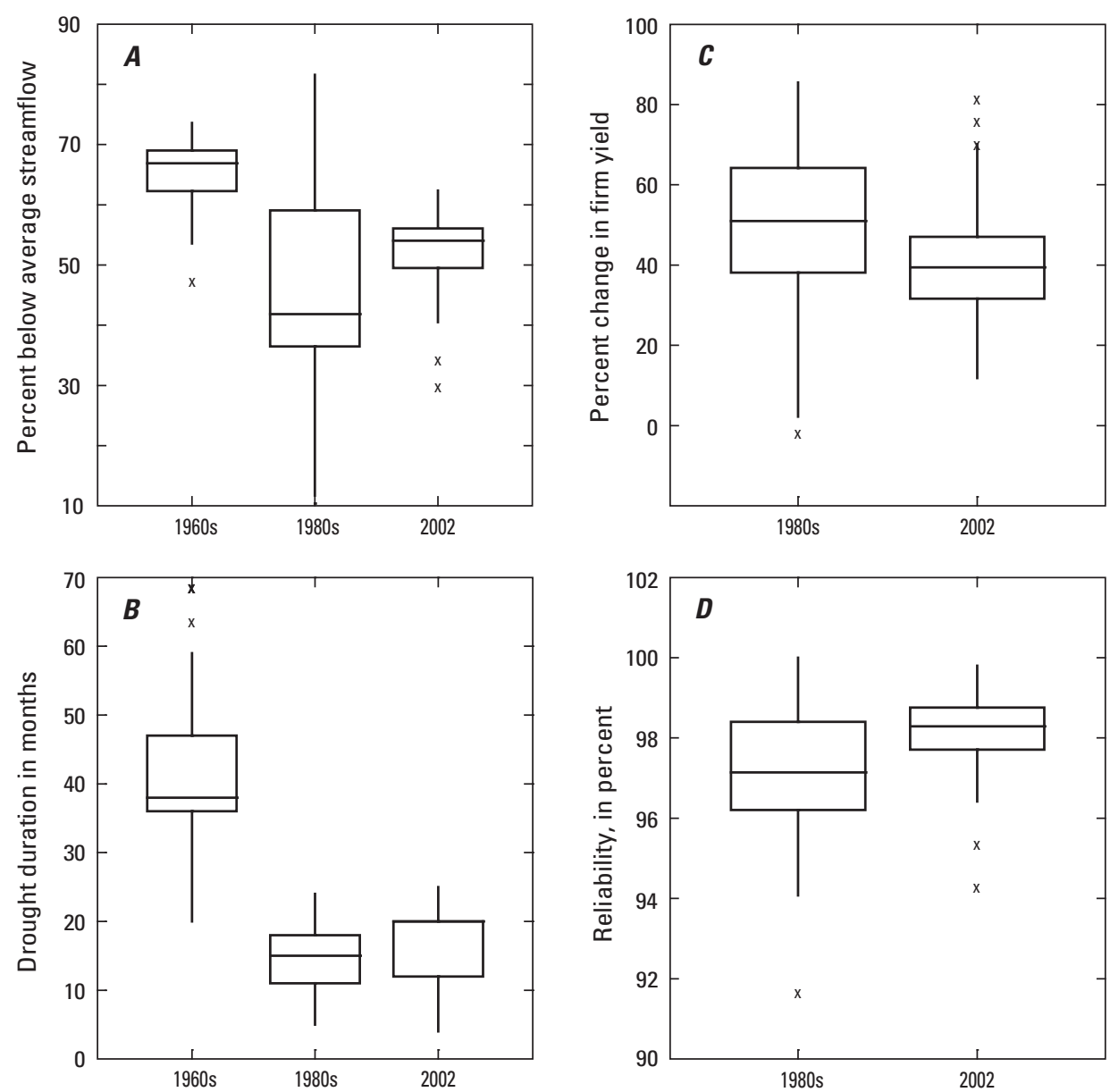

\section{EXPLANATION}

\section{Outlier}

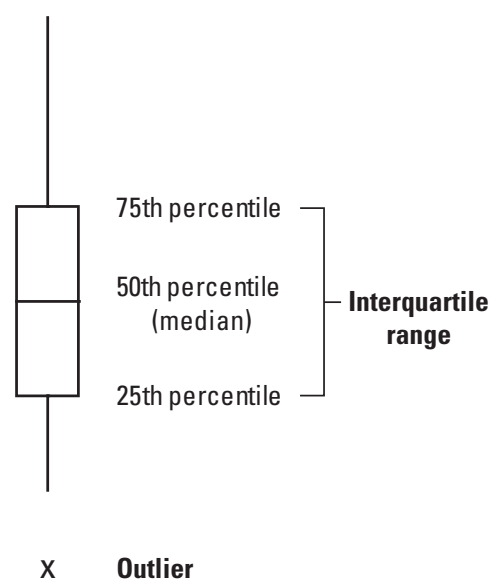

Figure 10. $A$, Percent below average streamflow and $B$, duration of droughts of the 1960s, 1980s, and 2002. $C$, Percent change in firm yield and $D$, reliability of firm yields when calculated with the droughts of the 1980s or 2002.

more intense during the drought period. Drought durations for both the 1980s and 2002 droughts ranged from 4 to 25 months. Reservoirs underwent a wide range of drought intensities in the 1980 s with average streamflows 12 to 82 percent below normal levels. Some reservoirs underwent two separate droughts in the 1980s, one at the beginning of the decade and one at the end. For the purposes of this study, the most severe of the two 1980s droughts was used. Streamflows were -30 to 62 percent below average levels during the 2002 drought.

The sensitivity of reservoir firm yield to drought severity was examined by calculating the firm yield using three different drought periods: 1960s, 1980s and 2001-2002 droughts. In order to calculate the firm yield using a drought from the 1980 s or 2002 , the FYE model was modified to consider only failures during the drought in question when calculating the firm yield. Reservoir failures that occurred either before or after the drought being studied were ignored by the model, and the firm yield was incremented until a failure occurred during the drought in question. This method produces results identical to the results of a simulation performed with a truncated historical record, as long as the reservoir returns to full pool before the drought period of interest. Reservoirs that did not return to full pool from one drought to the next were removed from the analysis. All reservoirs were run as single-reservoir systems for the purpose of this analysis.

For most reservoirs, the drought of the 1960s was the most severe and resulted in the lowest firm-yield estimate. Accord Pond in Hingham, Mass., was the only exception. For this reservoir, the firm yield estimated for a drought in 1981 was slightly lower than the yield estimated for the 1960 s drought. Overall, firm yields exceeded those during the 1960s drought by an average of 49 percent for the drought in the 1980s and 41 percent for the drought of 2001-2002. Individual reservoirs responded differently to these two droughts. Approximately three quarters of the reservoirs underwent more severe conditions during the 2001-2002 drought than in 
the drought of the 1980s, leading to lower firm-yield estimates for the 2001-2002 drought than for the 1980s drought.

Reservoirs operating at a firm yield computed for a less severe drought run the risk of failure should a more severe drought occur. The risk of failure for a particular reservoir can be characterized by the reservoir's reliability, resilience, and vulnerability. For each day of the simulated record, the reservoir state was classified as being either a failure or not. Reliability is the percentage of days in which the reservoir did not fail throughout the entire simulation. The reliability reflects the overall failure rate for the entire simulation period. For a given reliability, failures may occur in several long-duration events or many short-duration events. A failure event is the period of consecutive days a reservoir is in a failing state. The resilience of a reservoir and is defined as the average of the durations of all the failure events during the simulation. Reservoirs that recover quickly after a failure are more resilient and will have a shorter average failure duration. The magnitude of a failure is characterized by the yield deficit, which is the difference between the daily water demand and the daily amount that the reservoir is able to supply during a failure event.

In order to investigate the risk and magnitude of potential failures due to operating at a firm yield calculated for a drought other than the 1960s drought, the water-balance equation was solved using the estimated firm yields for the 1980s and 2002 droughts for each reservoir and allowing the reservoir to fail during other time periods in the simulation. These simulations were used to calculate reservoir reliability, average failure duration, and average yield deficit. Average reliabilities of reservoirs operating at the 1980s and 2002 firm yields were 97 and 98 percent, respectively (fig. 10). Average failure durations across reservoirs ranged from several days to several months. This information is useful for gaging the risk of failure should a drought more severe than any on record occur.

\section{Effect of Controlled Releases and Demand Management on Firm Yield}

Reservoirs that are managed solely to maintain storage and meet human water demand can affect downstream areas by depleting the amount of water available for ecological communities and disrupting natural streamflow patterns. When a river is impounded to create a drinking-water reservoir, water that would have flowed downstream is allocated to fill the reservoir or used to supply drinking water. During periods in which the reservoir is at full storage, any inflows that exceed water demand and natural reservoir outflows will spill to the downstream reach. During periods in which the reservoir water surface is below the spillway, downstream reaches will not receive any water.

Several recent studies have highlighted the effects of impoundments on Massachusetts streams and fish communities (Weiskel and others, 2010; Armstrong and others,
2010), and more consideration is being given to the environmental needs in the river reach downstream from a reservoir impoundment. One approach to mitigate the effects of impoundments on downstream reaches is to implement controlled releases from the reservoir into the stream either by manipulating spillway boards or through a release valve. Such releases would necessarily decrease the firm yield of a reservoir because they would increase the daily reservoir outflows, causing less water to be available for withdrawals. For reservoirs operating near their firm yield, meeting environmental flow requirements during a drought period could jeopardize the ability of the reservoir to meet drinking-water demands.

The effects of imposing instream-flow requirements may potentially be offset by implementing several management strategies. Water demand in Massachusetts typically peaks during summer months when water availability is the lowest. Reservoir storage may become depleted during these periods, leaving little or no water available for environmental flow releases. Demand-management scenarios such as nonessential summer water-use restrictions may help to lower water demand during these periods, allowing more water to be available for environmental flows. Another potential management strategy is to relax the definition of firm yield to allow reservoir operation at less than 100 percent reliability. Although this may put the reservoir at risk of occasional failure during severe droughts, this strategy may be feasible in towns that have emergency water sources or that can import water from other systems. Understanding the effects of management scenarios and controlled releases on firm yield is necessary for water-supply managers and regulators to evaluate the risks and tradeoffs between allocations of water for human versus environmental needs.

\section{Controlled-Release Scenarios}

The FYE can account for user-specified monthly release rates for downstream environmental flows. The choice of an appropriate controlled-release scenario for any particular reservoir in Massachusetts is an area of ongoing research. For illustrative purposes in this report, two hypothetical reservoir release scenarios were developed to test the effects of controlled releases on reservoir firm yield. For each month, a flow-duration curve was developed from the 44-year daily streamflow record. Monthly controlled releases for the two scenarios were set at the 10th- and 25th-percentile monthly flow volumes (appendix 4). For multiple-reservoir systems, reservoir releases were implemented at each reservoir in the system based on the respective streamflows estimated for each individual reservoir. Firm yields were calculated for each of the two controlled-release scenarios and compared with reported usage data from 2000 to 2004 (table 8). For the purposes of this study, all reservoirs were considered capable of releasing water downstream. This may not be possible in all systems because reservoirs may not have a release structure installed for this purpose. 
Table 8. Water usage and firm yields for reservoir systems in Massachusetts under various controlled-release, demand-management, with the Massachusetts Water Resources Authority in addition to reservoirs listed here.

[Mgal/d, million gallons per day; \%, percent; DPW, Department of Public Works]

Scenarios with no controlled releases

\begin{tabular}{|c|c|c|c|c|c|c|}
\hline Water supplier & Reservoir system & $\begin{array}{c}\text { Average } \\
\text { annual } \\
\text { usage } \\
2000-2004 \\
\text { (Mgal/d) }\end{array}$ & $\begin{array}{l}\text { Firm yield } \\
\text { with no-fail } \\
\text { criteria and } \\
\text { no demand } \\
\text { management } \\
\text { (Mgal/d) }\end{array}$ & $\begin{array}{l}\text { Firm yield } \\
\text { with no-fail } \\
\text { criteria and } \\
\text { demand } \\
\text { management } \\
\text { (Mgal/d) }\end{array}$ & $\begin{array}{l}\text { Firm yield } \\
\text { with } 1 \% \text { fail- } \\
\text { ure criteria, } \\
\text { no demand } \\
\text { management } \\
\text { (Mgal/d) }\end{array}$ & $\begin{array}{c}\text { Firm yield } \\
\text { with } 1 \% \text { fail- } \\
\text { ure criteria } \\
\text { and demand } \\
\text { management } \\
\text { (Mgal/d) }\end{array}$ \\
\hline Amherst Water Department & Amethyst Brook Intake system & 0.83 & 0.87 & 0.93 & 1.19 & 1.36 \\
\hline Amherst Water Department & Atkins Reservoir & 0.83 & 1.16 & 1.23 & 1.25 & 1.40 \\
\hline Ashburnham/Winchendon & Upper Naukeag Lake & 1.06 & 1.72 & 1.81 & 1.88 & 1.98 \\
\hline Concord Water Department & Nagog Pond & 0.09 & 0.86 & 1.02 & 0.96 & 1.27 \\
\hline Danvers Water Department & Middleton Pond system & 3.09 & 2.79 & 3.06 & 3.14 & 3.40 \\
\hline Fall River Water Department & North Watuppa system & 12.89 & 18.20 & 19.05 & 20.01 & 20.69 \\
\hline Fitchburg Water Department & Lovell Reservoir system & 0.18 & 2.77 & 2.89 & 3.20 & 3.43 \\
\hline Fitchburg Water Department & Meetinghouse system & 2.71 & 5.61 & 6.33 & 6.30 & 6.63 \\
\hline Fitchburg Water Department & Wachusett Lake & 0.35 & 1.08 & 1.18 & 1.24 & 1.36 \\
\hline Greenfield Water Department & Green River & 0.52 & 0.42 & 0.42 & 1.49 & 1.49 \\
\hline Greenfield Water Department & Leyden Glen Reservoir & 0.57 & 0.69 & 0.69 & 1.12 & 1.17 \\
\hline Hingham/Hull (Aquarian Water Company) & Accord Pond & 0.59 & 0.66 & 0.77 & 0.75 & 0.87 \\
\hline Hinsdale Water Department & Belmont Reservoir & 0.15 & 0.12 & 0.13 & 0.15 & 0.16 \\
\hline Lee Water Department & Schoolhouse Reservoir & 0.51 & 0.84 & 0.89 & 1.14 & 1.23 \\
\hline Lee Water Department & Upper (Leahey) Reservoir & 0.54 & 0.59 & 0.64 & 0.67 & 0.73 \\
\hline Leicester (Cherry Valley and Rochdale Water) & Henshaw Pond & 0.26 & 0.35 & 0.36 & 0.43 & 0.46 \\
\hline Leominster DPW-Water Division & Distributing Reservoir system & 0.87 & 0.58 & 0.66 & 0.66 & 0.78 \\
\hline Leominster DPW-Water Division & Fall Brook Reservoir & 0.88 & 0.84 & 0.90 & 0.98 & 1.10 \\
\hline Leominster DPW-Water Division & Simonds Pond system & 2.32 & 2.26 & 2.43 & 2.77 & 2.99 \\
\hline Lincoln Water Department & Flints Pond & 0.38 & 0.59 & 0.64 & 0.68 & 0.73 \\
\hline Marlborough DPW-Water and Sewer Division & Millham system & 1.40 & 1.83 & 1.99 & 2.15 & 2.32 \\
\hline Milford Water Company ${ }^{1}$ & Echo Lake & 1.40 & 1.12 & 1.20 & 1.42 & 1.42 \\
\hline North Brookfield Water Department & Doane Pond system & 0.42 & 0.71 & 0.74 & 0.80 & 0.86 \\
\hline Pittsfield Water Department & Ashley Lake & 0.21 & 1.37 & 1.41 & 1.82 & 1.90 \\
\hline Pittsfield Water Department & Cleveland Reservoir & 7.96 & 9.18 & 9.47 & 10.53 & 11.17 \\
\hline Pittsfield Water Department & Farnham system & 2.23 & 2.51 & 2.66 & 2.98 & 3.21 \\
\hline Pittsfield Water Department & Upper Sackett Reservoir & 0.08 & 0.50 & 0.55 & 0.58 & 0.64 \\
\hline Scituate Water Department ${ }^{3}$ & Main Reservoir & 0.61 & 0.63 & 0.70 & 0.81 & 0.94 \\
\hline South Deerfield Water Supply District & Whately system & 0.68 & 1.07 & 1.11 & 1.09 & 1.26 \\
\hline Southbridge Water Department & Hatchet Brook system & 1.69 & 2.78 & 2.87 & 3.28 & 3.57 \\
\hline Springfield Water Department & Cobble Mountain system & 36.57 & 42.70 & 45.14 & 45.42 & 48.83 \\
\hline Wakefield Water Department & Crystal Lake & 0.28 & 0.40 & 0.44 & 0.47 & 0.51 \\
\hline West Springfield Water Department & Bearhole Reservoir & 0.73 & 1.40 & 1.53 & 1.72 & 1.99 \\
\hline Westborough Water Department & Sandra Pond & 0.67 & 0.80 & 0.86 & 0.89 & 0.95 \\
\hline Westfield Water Department & Granville Reservoir & 2.47 & 3.03 & 3.18 & 3.66 & 3.95 \\
\hline Westfield Water Department & Montgomery Reservoir & $\mathbf{0 . 0 0}$ & 1.13 & 1.17 & 1.35 & 1.43 \\
\hline Winchester Water Department & South Reservoir system & 1.10 & 1.01 & 1.07 & 1.11 & 1.20 \\
\hline Worcester Water Department ${ }^{2}$ & Holden Pond system & 23.87 & 17.15 & 17.64 & 18.87 & 20.38 \\
\hline
\end{tabular}

\footnotetext{
${ }^{1}$ Firm yield for Echo Lake includes water from the Charles River diversion.
}

${ }^{2}$ Firm yield for the Worcester reservoir system is subject to additional uncertainty due to complex water routing between reservoirs.

${ }^{3}$ Firm yield for Scituate does not include Old Oaken Bucket Pond. 
and reliability scenarios. Marlborough, Leominster, Wakefield, and Worcester have water-supply connections

Scenarios with 10th-percentile monthly flow releases Scenarios with 25th-percentile monthly flow releases

\begin{tabular}{|c|c|c|c|c|c|c|c|}
\hline $\begin{array}{c}\text { Firm yield } \\
\text { with no-fail } \\
\text { criteria and } \\
\text { no demand } \\
\text { management } \\
\text { (Mgal/d) }\end{array}$ & $\begin{array}{l}\text { Firm yield } \\
\text { with no-fail } \\
\text { criteria and } \\
\text { demand } \\
\text { management } \\
\text { (Mgal/d) }\end{array}$ & $\begin{array}{c}\text { Firm yield } \\
\text { with } 1 \% \text { fail- } \\
\text { ure criteria, } \\
\text { no demand } \\
\text { management } \\
\text { (Mgal/d) }\end{array}$ & $\begin{array}{c}\text { Firm yield } \\
\text { with } 1 \% \text { fail- } \\
\text { ure criteria } \\
\text { and demand } \\
\text { management } \\
\text { (Mgal/d) }\end{array}$ & $\begin{array}{l}\text { Firm yield } \\
\text { with no-fail } \\
\text { criteria and } \\
\text { no demand } \\
\text { management } \\
\text { (Mgal/d) }\end{array}$ & $\begin{array}{l}\text { Firm yield } \\
\text { with no-fail } \\
\text { criteria and } \\
\text { demand } \\
\text { management } \\
\text { (Mgal/d) }\end{array}$ & $\begin{array}{c}\text { Firm yield } \\
\text { with } 1 \% \text { fail- } \\
\text { ure criteria, } \\
\text { no demand } \\
\text { management } \\
\text { (Mgal/d) }\end{array}$ & $\begin{array}{c}\text { Firm yield } \\
\text { with } 1 \% \text { fail- } \\
\text { ure criteria } \\
\text { and demand } \\
\text { management } \\
\text { (Mgal/d) }\end{array}$ \\
\hline 0.34 & 0.46 & 0.71 & 1.01 & 0.56 & 0.79 & 0.63 & 0.86 \\
\hline 0.81 & 0.86 & 0.90 & 0.99 & 0.60 & 0.63 & 0.68 & 0.75 \\
\hline 1.44 & 1.52 & 1.58 & 1.66 & 1.22 & 1.29 & 1.35 & 1.42 \\
\hline 0.71 & 0.84 & 0.79 & 1.02 & 0.62 & 0.73 & 0.68 & 0.85 \\
\hline 2.01 & 2.18 & 2.21 & 2.39 & 1.41 & 1.53 & 1.57 & 1.70 \\
\hline 13.39 & 14.01 & 14.78 & 15.27 & 10.80 & 11.30 & 11.93 & 12.37 \\
\hline 2.12 & 2.21 & 2.49 & 2.66 & 1.64 & 1.71 & 2.03 & 2.17 \\
\hline 4.32 & 4.79 & 4.83 & 5.21 & 3.54 & 3.89 & 4.02 & 4.35 \\
\hline 0.77 & 0.85 & 0.91 & 1.00 & 0.61 & 0.67 & 0.73 & 0.79 \\
\hline 0.00 & 0.00 & 0.00 & 0.00 & 0.00 & 0.00 & 0.00 & 0.00 \\
\hline 0.21 & 0.21 & 0.45 & 0.48 & 0.00 & 0.00 & 0.00 & 0.00 \\
\hline 0.50 & 0.57 & 0.59 & 0.67 & 0.40 & 0.45 & 0.47 & 0.53 \\
\hline 0.06 & 0.06 & 0.08 & 0.08 & 0.02 & 0.02 & 0.04 & 0.04 \\
\hline 0.43 & 0.45 & 0.62 & 0.66 & 0.17 & 0.17 & 0.25 & 0.27 \\
\hline 0.47 & 0.52 & 0.56 & 0.60 & 0.41 & 0.45 & 0.49 & 0.53 \\
\hline 0.19 & 0.19 & 0.24 & 0.27 & 0.08 & 0.12 & 0.15 & 0.17 \\
\hline 0.37 & 0.44 & 0.47 & 0.55 & 0.27 & 0.29 & 0.36 & 0.40 \\
\hline 0.61 & 0.65 & 0.71 & 0.81 & 0.46 & 0.49 & 0.55 & 0.62 \\
\hline 1.60 & 1.72 & 1.64 & 1.92 & 1.21 & 1.31 & 1.25 & 1.53 \\
\hline 0.45 & 0.49 & 0.52 & 0.56 & 0.36 & 0.39 & 0.42 & 0.45 \\
\hline 1.06 & 1.14 & 1.25 & 1.35 & 0.60 & 0.64 & 0.79 & 0.85 \\
\hline 0.90 & 0.96 & 1.05 & 1.16 & 0.72 & 0.77 & 0.85 & 0.94 \\
\hline 0.55 & 0.57 & 0.63 & 0.68 & 0.45 & 0.47 & 0.52 & 0.56 \\
\hline 0.89 & 0.92 & 1.25 & 1.35 & 0.61 & 0.63 & 0.90 & 0.98 \\
\hline 5.99 & 6.30 & 7.22 & 7.74 & 4.15 & 4.35 & 5.34 & 5.75 \\
\hline 1.92 & 2.03 & 2.20 & 2.38 & 1.60 & 1.69 & 1.87 & 2.03 \\
\hline 0.34 & 0.37 & 0.39 & 0.44 & 0.24 & 0.26 & 0.28 & 0.32 \\
\hline 0.13 & 0.13 & 0.51 & 0.61 & 0.00 & 0.00 & 0.12 & 0.14 \\
\hline 0.80 & 0.89 & 0.83 & 1.04 & 0.41 & 0.50 & 0.45 & 0.55 \\
\hline 2.31 & 2.38 & 2.76 & 2.97 & 2.00 & 2.05 & 2.33 & 2.46 \\
\hline 33.01 & 34.80 & 35.54 & 37.97 & 27.36 & 28.74 & 29.55 & 31.21 \\
\hline 0.24 & 0.27 & 0.29 & 0.32 & 0.14 & 0.16 & 0.17 & 0.19 \\
\hline 0.78 & 0.87 & 0.94 & 1.05 & 0.00 & 0.00 & 0.21 & 0.25 \\
\hline 0.55 & 0.59 & 0.62 & 0.66 & 0.37 & 0.40 & 0.42 & 0.46 \\
\hline 1.79 & 1.87 & 2.35 & 2.55 & 0.91 & 0.96 & 1.48 & 1.59 \\
\hline 0.60 & 0.63 & 0.80 & 0.89 & 0.24 & 0.24 & 0.43 & 0.49 \\
\hline 0.82 & 0.86 & 0.91 & 0.98 & 0.70 & 0.74 & 0.75 & 0.82 \\
\hline 11.34 & 11.74 & 12.37 & 13.61 & 3.57 & 9.20 & 9.82 & 10.66 \\
\hline
\end{tabular}


Controlled releases for the two scenarios lowered firm yields by an average of 35 percent for the 10th-percentile flow-release scenario and 55 percent for the 25th-percentile flow-release scenario. Of the 38 systems studied, 16 were able to meet or marginally meet their current (2000 to 2005) usage requirements while releasing monthly 10 th-percentile flows, and 11 were able to meet or marginally meet their current usage while releasing 25th-percentile flows. Because yearto-year usage in a system can vary, systems in which average usage was within 10 percent of the firm yield of a particular scenario may be operating within the firm yield in some years but above the firm yield in other years. These reservoirs are listed as marginally operating within the firm yield (table 8).

\section{Summer Water-Demand Management}

Outdoor summer water-use restrictions are often implemented by water-resources managers as a way to conserve water during drought periods. Water usage peaks during summer months due to outdoor water use, such as irrigation, residential-lawn watering, or filling of swimming pools. Some communities have alternative water sources such as groundwater wells or water supply connections with the Massachusetts Water Resources Authority that can lessen the water demand burden on the reservoir system. For communities without these resources, summer water-use restrictions may be used to conserve water and maintain adequate reservoir levels. Outdoor summer water-use restrictions decrease the summer water demand and often go into effect when a reservoir falls below a certain level. Demand-reduction scenarios can be implemented in several stages as the reservoir falls to successively lower levels. The firm-yield-model code was altered to allow the user to specify a summer water-use-restriction scenario. This type of demand-management scenario can be specified by the user as a percentage by which to decrease demand during summer months (June-September) when the reservoir falls below a user-specified percentage of its total capacity. If reservoir storage falls below the percentage of total capacity specified in the demand-management scenario on any simulation day, and the simulation is in a summer month, the yield, $Q_{y}$, for that day is decreased by the percentage specified by the user. Simulation then continues as usual. When a multiple-reservoir system is simulated, the demand-management scenario is applied to all reservoirs in the system.

The amount of summer water-demand reductions that can be achieved by summer outdoor water restrictions is highly variable and depends on many factors such as climate, land use, and population density. Further study is needed to determine appropriate demand-reduction strategies for

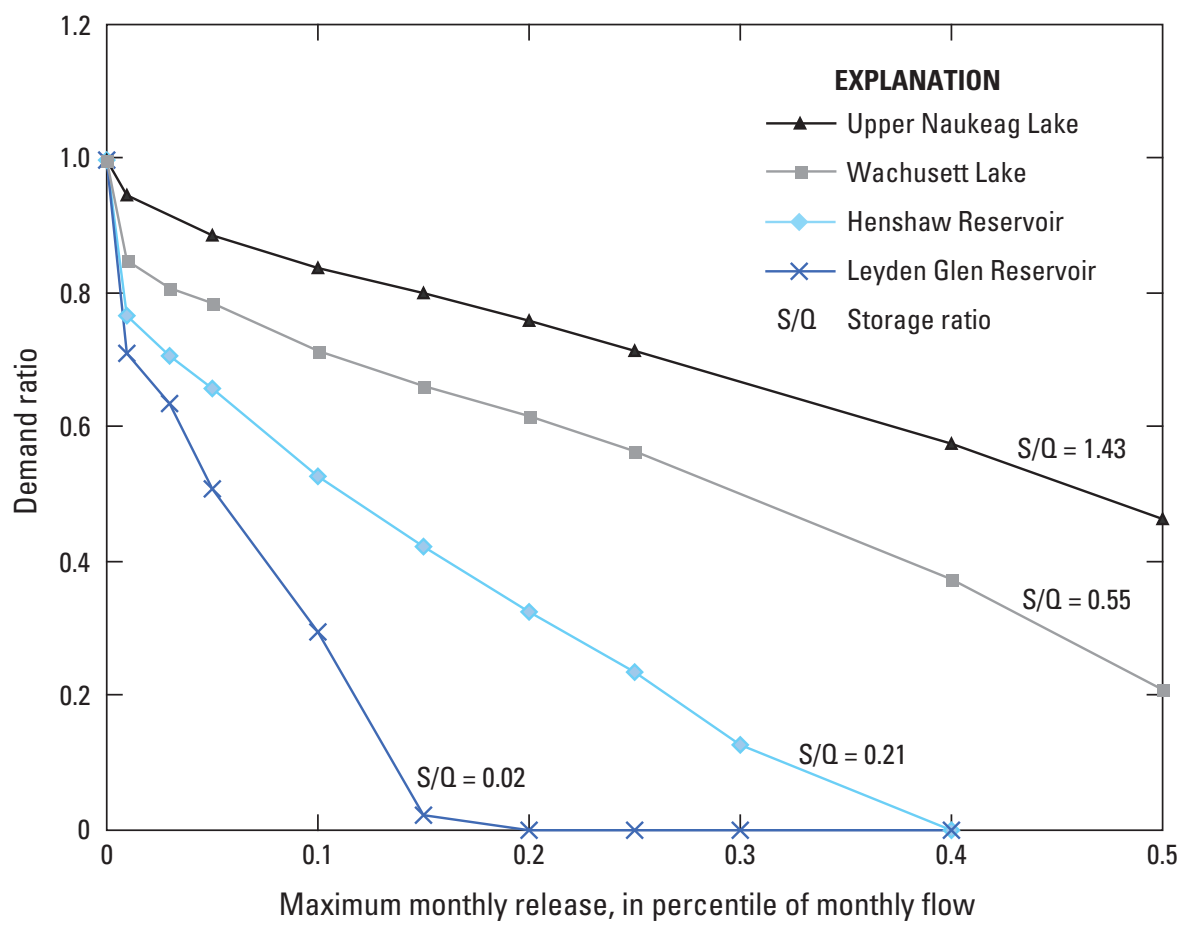

Figure 11. Maximum monthly releases as a percentile of longterm monthly flows that is possible at various demand ratios for four reservoirs of increasing storage ratio. 
Massachusetts reservoirs. For illustrative purposes in this report, the effect of implementing one hypothetical demandmanagement scenario was examined for the reservoir systems in this study. The demand-management scenario specified a 15-percent demand reduction when the reservoir fell to 60 percent of its maximum capacity, a 25 -percent demand reduction when the reservoir fell to 40 percent of its maximum capacity, and a 35-percent demand reduction when the reservoir fell to 20 percent of its total capacity. Reducing summer demand in this way increased firm yields by an average of 7 percent. By implementing this demand-reduction scenario, two of the six systems that are currently operating above the estimated firm yield were able to marginally meet demand requirements. In addition, this demand scenario enabled one additional reservoir system to support 25th-percentile flow releases and four additional systems to support 10th-percentile flow releases (table 8).

The amount of water available for downstream flow releases depends on the water demands of the reservoir and the storage ratio. The demand ratio is defined as the annual average water demand divided by the firm yield of the reservoir. For a given controlled-release rate, the maximum demand ratio possible for a reservoir may be calculated by dividing the firm yield calculated with controlled releases by the firm yield without releases. Reservoirs that are operating at or near their firm yield will have demand ratios close to 1 . These reservoirs have less water available for downstream flow releases because most of the available water in storage is being allocated to human use. Reservoirs with low storage ratios are also less able to support high levels of controlled releases. Reservoirs with low storage in relation to their average daily streamflow are more susceptible to variability in daily streamflow and have proportionally less water available in storage during even minor periods of low flow. The maximum controlled releases (as percentiles of long-term monthly flows) that are possible at various demand ratios for selected reservoirs with increasing storage capacity are shown in figure 11 .

\section{Reducing Reservoir Reliability Requirements}

Reservoir reliability is set by the user by specifying the maximum number of days during the simulation when the reservoir is allowed to fail. Reliability is calculated as the percentage of days when the reservoir did not fail during the simulation. In order to run the FYE at a reliability of less than 100 percent, the model code was modified to allow the simulation to run through a failing state. When calculating the daily water balance, the model will first solve the waterbalance equation without allocating for yield. If reservoir storage at this point is sufficiently high to fully satisfy daily demand, then the usage term is subtracted, and simulation continues as normal. If the usable storage at this point is not sufficient to satisfy daily demand, then all the available water in storage is allocated to yield, bringing available storage to zero. The reservoir is considered to be failing in this situation because the amount of water supplied by the reservoir is less than the full demand volume. After the full 44-year simulation is completed, the number of failure days is totaled. Yield is incrementally increased until the number of days of reservoir failures equals the amount specified by the user. The failures may occur in many short-duration events or a small number of long-duration events. The total number of failure events and the average duration of failure periods are reported for each reservoir simulation. In addition, the FYE also calculates and reports the average yield deficit. These failure statistics can help water managers gage the risks and potential costs of employing a strategy that includes relaxing the no-fail operating criterion of a reservoir. For multiplereservoir systems, the reliability set by the user is applied to all reservoirs in the system.

In order to examine the effect of relaxing the no-fail criterion, simulations of the reservoir systems in this study were run using a 99-percent reliability criterion (fig. 12). This criterion allows for a total of 160 allowable failures during the 44-year simulation period. Relaxing the reliability criterion increased yields by an average of 25 percent. Under this scenario, estimated yields are sufficient to meet demand at all but three systems in the study. In addition, relaxing the reliability criterion enables two additional systems to support 25th-percentile flow releases and nine additional systems to support 10th-percentile flow releases; these systems could not otherwise support releases under the no-fail scenario.

\section{Tradeoffs Between Demand Management, Controlled Releases, and Reliability}

Implementing downstream release flows, implementing demand-management strategies, or operating the reservoir at a lower reliability all have risks and benefits associated with them. In determining the best possible strategy for any given reservoir system, a water manager may use any combination of these three strategies in order to maximize the reservoir yield at the lowest cost. Tradeoff curves can be constructed to examine the effect of different strategies on the firm yield. For a given reservoir, a family of tradeoff curves can be constructed showing the different combinations of controlled releases, reliability criteria, and demand-management strategies that can be used to achieve a target yield. Sample tradeoff curves for Upper Leahey Reservoir are shown in figure 13. On the basis of reported water usage for 2000-2004, Upper Leahey would be able to support only very minimal environmental flow releases without implementing some sort of demand-management strategy. In order to achieve controlled releases at the 10th percentile of monthly flows, a more severe outdoor summer water-use restriction scenario would need to be implemented than the one tested here. 


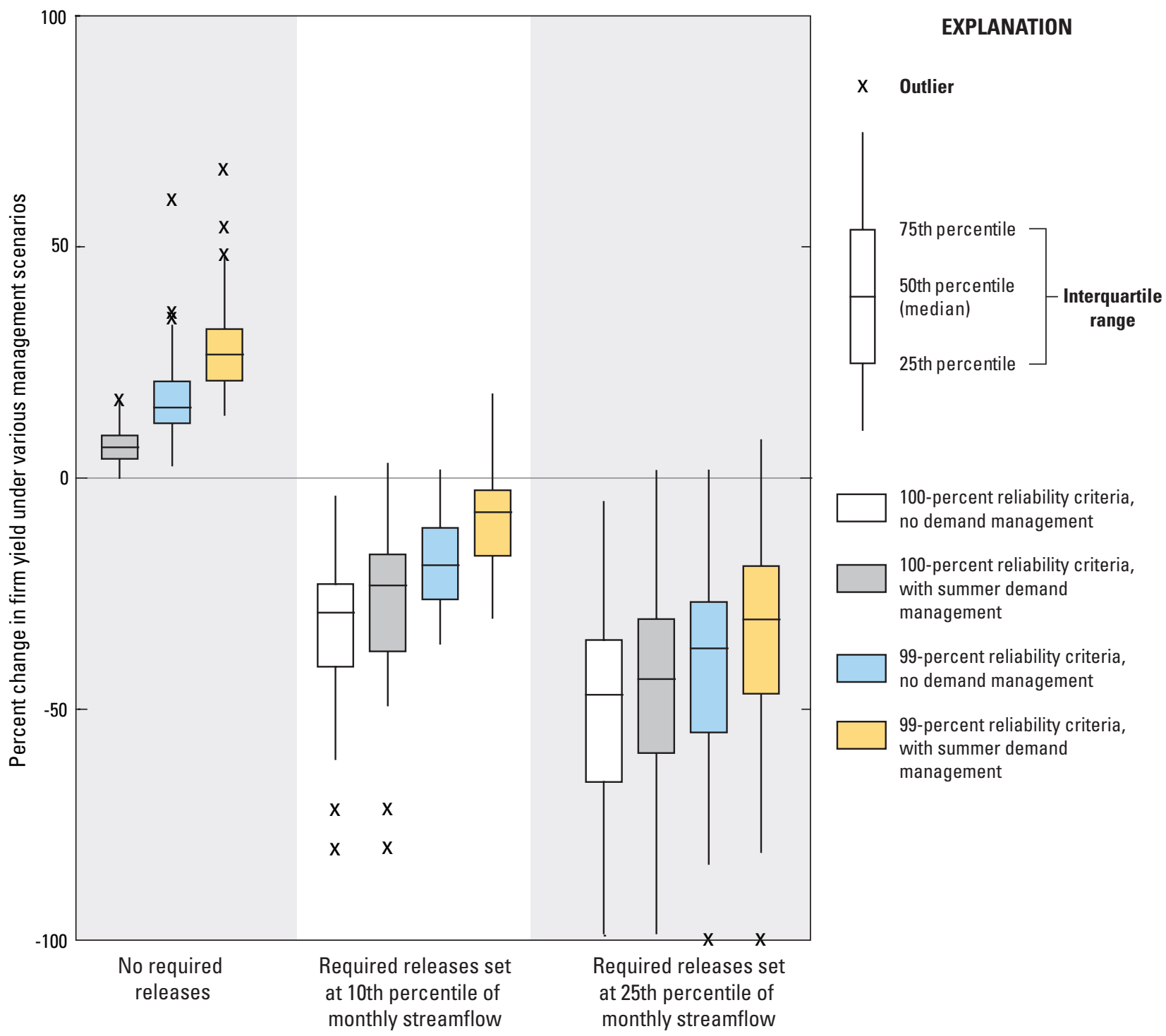

Figure 12. Percent changes of firm yield for Massachusetts reservoirs under various management scenarios. 


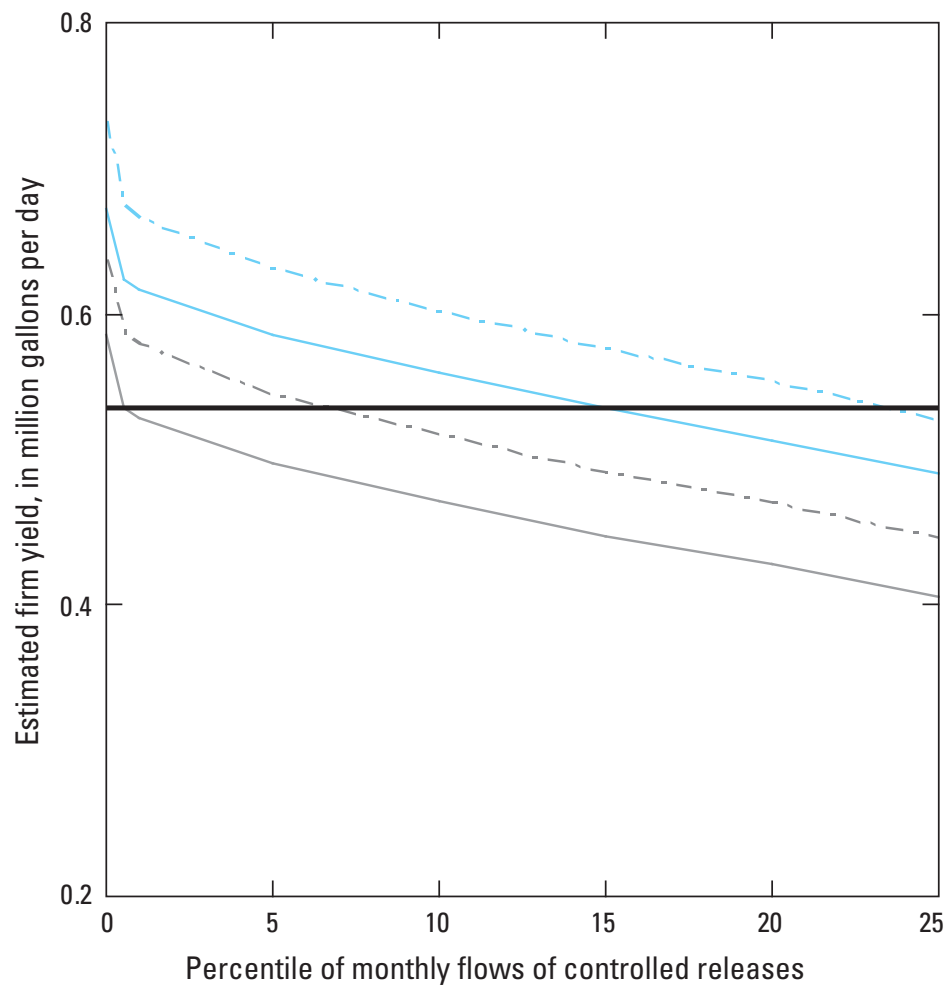

EXPLANATION

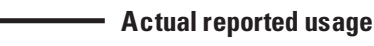

100-percent reliability, no demand management

$-\cdot-\cdot$ 100-percent reliability with demand management

99-percent reliability, no demand management

99-percent reliability with demand management

Figure 13. Tradeoff curves depicting the estimated yield in relation to controlled releases under various management scenarios for Upper Leahy Reservoir, in Lee, Massachusetts.

\section{Summary and Conclusions}

Procedures for determining the firm yield of a reservoir, which were previously developed, were further refined and implemented for 38 reservoir systems in Massachusetts, including 25 single- and multiple-reservoir systems that were examined in previous studies and 13 additional reservoir systems. Changes to the Firm-Yield Estimator (FYE) model include simulation of the 44-year historical record by daily time steps; the incorporation of daily input data for streamflow, precipitation, and evaporation; refinements to the groundwater simulation; and enhanced scenario-testing capabilities. This study documents the procedural refinements to the model, examines sources of uncertainty in the estimated firm yields, and demonstrates the use of the model to assess the feasibility of controlled releases under several example management scenarios. Because of these refinements, estimates of firm yield based on previous versions of the FYE are no longer considered valid.
Uncertainty in the FYE model comes from many sources, including errors in the input data for streamflow, precipitation, evaporation, and stage-storage relations. Reservoirs that overlie substantial sand and gravel deposits have additional uncertainty in firm-yield estimates because of uncertainty in estimating groundwater inflows and outflows to the reservoir. In addition, uncertainty in firm-yield estimates may arise from other factors, such as seepage or reservoir-operation details, that are not accounted for in the FYE. An analytical method to estimate overall uncertainty in the FYE model was not determined; however, the sensitivity of the model to errors in daily streamflow and stage-storage relations was examined. Because the lowest streamflows may be underestimated by the SYE, firm yields estimated by the FYE may be conservative. A Monte Carlo simulation showed that, on average, firm yields increased 1 to 10 percent after accounting for errors in daily streamflows. Errors in firm yields can also arise from errors in the stage-storage and volume calculations as a result of spatially imprecise bathymetric data. Experiments on three 
reservoirs showed that bathymetric data that are sampled too sparsely can result in an underestimation of reservoir volume and firm yield. Bathymetric surveys with measurement densities of less than 20 points per acre or transects spaced more than 30 to 60 meters apart resulted in underestimation of reservoir volume and firm-yield estimates by 5 percent or more.

Reservoirs in contact with sand and gravel may receive water flows from and may discharge to groundwater sources. In a previous study, equations were developed and implemented to estimate the magnitude of groundwater contributions to the reservoir water balance on the basis of changes in reservoir storage at each time step. The parameterization of these equations was refined in this study in order to allow the scaling factor to be adjusted on the basis of the changing reservoir stage. This eliminated instabilities in the groundwater-equation algorithm that arose during low-storage periods. Validation of the groundwater parameters at one reservoir indicated that parameter estimation from published maps is highly uncertain.

Firm yields estimated using the historical record are sensitive to the severity of droughts during the simulation periods. For this study, a 44-year historical record, which includes the most severe drought on record, was used for firm-yield estimation. Firm yields based on this drought may not be adequate to protect against failures should a more severe drought occur in the future. The sensitivity of firm yields to drought severity was examined by estimating firm yields based on three droughts during the simulation period. Firm yields based on droughts in the 1980s and 2002 exceeded those during the 1960s drought by an average of 49 and 41 percent, respectively; however, operating reservoirs at these yields led to average failure rates of 2 to 3 percent when applied over the entire simulation period.

Because of concern over water availability for ecological needs in Massachusetts streams, regulators may wish to examine the effects of regular releases from drinkingwater reservoirs to enhance instream flow downstream from impoundments. An analysis of two controlled-release scenarios showed that reservoirs with a large storage ratio (reservoir capacity divided by mean streamflow) and low demand ratio (average annual water demand as a percentage of the annual firm yield) were able to support the highest levels of flow releases without sacrificing yield for human use. Roughly half the reservoir systems studied were able to support minimal monthly flow releases equal to the monthly 10th-percentile daily flow. Reservoirs can increase their daily yield by implementing summer water-use restrictions or by relaxing the no-fail criterion of the firm yield. One scenario involving hypothetical summer water-use restrictions and another involving a 1-percent failure criterion were tested. The scenario with summer water-use restrictions that was tested led to an average increase of 7 percent in the firm yield, which was generally not sufficient to enable controlled releases from a reservoir that was otherwise unable to support them. Relaxing the reservoir reliability to 99 percent led to an average increase of 25 percent in reservoir firm yield but also left the reservoir vulnerable to failures. Reservoir operators can weigh the relative risks and benefits of various management strategies using tradeoff curves and statistics for failure magnitude and duration.

\section{References Cited}

Archfield, S.A., and Carlson, C.S., 2006, Ground-water contributions to reservoir storage and the effect on estimates of firm yield for reservoirs in Massachusetts: U.S. Geological Survey Scientific Investigations Report 2006-5045, 27 p.

Archfield, S.A., Vogel, R.M., Steeves, P.A., Brandt, S.L., Weiskel, P.K., and Garabedian, S.P., 2010, The Massachusetts Sustainable-Yield Estimator-A decisionsupport tool to assess water availability at ungaged stream locations in Massachusetts: U.S. Geological Survey Scientific Investigations Report 2009-5227, 41 p., plus CD-ROM.

Armstrong, D.S., Richards, T.A., and Brandt, S.L., 2010, Preliminary assessment of factors influencing riverine fish communities in Massachusetts: U.S. Geological Survey Open-File Report 2010-1139, 43 p.

Environmental Systems Research Institute, Inc. (ESRI), 1994, ARC/INFO user guides, version7: Redlands, Calif., Environmental Systems Research Institute, Inc., 626 p.

Fennessey, N.M., 1995, The sensitivity of reservoir-yield estimates to model time step surface-moisture fluxes: Journal of Water Resources Planning and Management, v. 121, no. 4, July/August 1995, p. 310-317.

Hargreaves, G.H., and Samni, Z.A., 1982, Estimation of potential evapotranspiration: Journal of Irrigation and Drainage Division, Proceedings of the American Society of Civil Engineers, v. 108, p. 223-230.

Kruizinga, S., and Yperlaan, G.J., 1978, Spatial interpolation of daily totals of rainfall: Journal of Hydrology, v. 36, p. 65-73.

Massachusetts Department of Environmental Protection 1996, Estimating the firm yield of a surface water reservoir supply system in Massachusetts-A guidance document, version 1.0: Prepared by N. Fennessey for the Massachusetts Department of Environmental Protection, Office of Watershed Management, variously paginated.

Massachusetts Department of Environmental Protection 2000, Firm yield estimator, version 1.0, software documentation: Prepared by Cambridge Environmental, Inc., for the Massachusetts Department of Environmental Protection, Office of Watershed Management, variously paged. 
Metcalf \& Eddy, Inc., 1997, Water, aster plan and analysis of reservoir yield: Submitted to Milford Water Company, March 1997, variously paginated.

Nash, J.E., and Sutcliffe, J.V., 1970, River flow forecasting through conceptual models, part I-A discussion of principles: Journal of Hydrology, v. 10, no. 3, p. 282-290.

Office of Geographic and Environmental Information (Mass-GIS), 2004, Commonwealth of Massachusetts Executive Office of Environmental Affairs, 1:25,000-scale surficial geology digital data layer for sand and gravel deposits in Massachusetts, accessed January 15, 2009, at http://www.mass.gov/mgis/ftpsg.htm

Office of Geographic and Environmental Information (Mass-GIS), 2005, Commonwealth of Massachusetts Executive Office of Environmental Affairs, 1:5,000-scale Color Ortho Imagery of Massachusetts, accessed January 15, 2009, at http://www.mass.gov/mgis/ftpsg.htm

Rorabaugh, M.I., 1964, Estimating changes in bank storage and ground-water contribution to streamflow: International Association of Scientific Hydrology, Publication 63, p. $432-441$.

Waldron, M.C., and Archfield, S.A., 2006, Factors affecting firm yield and the estimation of firm yield for selected streamflow-dominated drinking-water-supply reservoirs in Massachusetts: U.S. Geological Survey Scientific Investigations Report 2006-5044, 39 p.

Weiskel, P.K., Brandt, S.L., DeSimone, L.A., Ostiguy, L.J., and Archfield, S.A., 2010, Indicators of streamflow alteration, habitat fragmentation, impervious cover, and water quality for Massachusetts stream basins: U.S. Geological Survey Scientific Investigations Report 20095272, 70 p., plus CD-ROM.

Wilson, G.L., and Richards, J.M., 2006, Procedural documentation and accuracy assessment of bathymetric maps and area/capacity tables for small reservoirs: U.S. Geological Survey Scientific Investigations Report 20065208, 24 p., plus oversize figures. 
THIS PAGE INTENTIONALLY LEFT BLANK 


\section{Appendix 1. Hypsographic Data}

Tables of reservoir storage, surface area, stage, and length of reservoir perimeter in contact with sand and gravel for each reservoir included in this report that were not included in previous firmyield reports are located on the CD-ROM in the folder titled HypsographicData.

\section{CD-ROM}

\section{[In pocket]}

\section{Contents}

Amethyst Brook Intake Reservoir

Accord Pond

Atkins Reservoir

Borden Brook Reservoir

Cobble Mountain Reservoir

Copicut Reservoir

Crystal Lake

Echo Lake

Flints Pond

Hawley Reservoir

Hill Reservoir

Holden Reservoir \#1

Holden Reservoir \#2

Kendall Reservoir

Kettle Brook Reservoir \#1

Kettle Brook Reservoir \#2

Kettle Brook Reservoir \#3

Kettle Brook Reservoir \#4

Lynde Brook Reservoir

Main Reservoir

Nagog Reservoir

North Watuppa Reservoir

Quinapoxet Reservoir

Sandra Pond

Upper Naukeag Lake 
THIS PAGE INTENTIONALLY LEFT BLANK 


\section{Appendix 2. Bathymetric Maps}

Bathymetric maps of all reservoirs included in this report that were not included in previous firm-yield reports are located on the CD-ROM in the folder titled BathymetricMaps.

\section{CD-ROM}

\section{[In pocket]}

\section{Contents}

Amherst Water Department

Aquarian Water Company, Hingham-Hull, Massachusetts

Ashburnham-Winchendon Joint Water Board

Concord Water Department

Fall River Water Division

Lincoln Water Department

Milford Water Company

Scituate Water Department

Springfield Water and Sewer Commission

Wakefield Water Department

Westborough Water Department

Worcester Water Department 
THIS PAGE INTENTIONALLY LEFT BLANK 


\section{Appendix 3. Reservoir-System Diagrams}

Schematic diagrams of all reservoir systems included in this report that were not included in previous firm-yield reports are located on the CD-ROM in the folder titled SystemDiagrams.

\section{CD-ROM}

\section{[In pocket]}

\section{Contents}

Accord Pond Bottom Elevation, Volume, and Surface Area

Hingham/Hull (Aquarian Water Company)

Amethyst Brook Intake

Amherst Water Department

Atkins Reservoir

Amherst Water Department

Borden Brook Reservoir

Springfield Water Department

Cobble Mountain Reservoir

Springfield Water Department

Copicut Reservoir

Fall River Water Department

Crystal Lake

Wakefield Water Department

Echo Lake

Milford Water Company

Flints Pond

Lincoln Water Department

Hawley Reservoir

Amherst Water Department

Hill Reservoir

Amherst Water Department

Holden Reservoir \#1

Worcester Water Department

Holden Reservoir \#2

Worcester Water Department

Kendall Reservoir

Worcester Water Department 
Kettle Brook Reservoir \#1

Worcester Water Department

Kettle Brook Reservoir \#2

Worcester Water Department

Kettle Brook Reservoir \#3

Worcester Water Department

Kettle Brook Reservoir \#4

Worcester Water Department

Lynde Brook Reservoir

Worcester Water Department

Main Reservoir

Scituate Water Department

Nagog Pond

Concord Water Department

North Watuppa Reservoir

Fall River Water Department

Pine Hill Reservoir

Worcester Water Department

Quinapoxet Reservoir

Worcester Water Department

Sandra Pond

Westborough Water Department

Upper Naukeag Lake

Ashburnham/Winchendon Joint Water Board 


\section{Appendix 4. Monthly Percentile Streamflows}

Tables of monthly 10th and 25th percentile reservoir inflows that were used for management scenarios in this report are located on the CD-ROM in the folder titled MonthlyFlowTables.

CD-ROM

[In pocket]

\section{Tables}

Monthly 10th Percentile of Streamflows Estimated Using the Sustainable Yield Estimator for Reservoirs in Massachusetts, 1960-2004

Monthly 25th Percentile of Streamflows Estimated Using the Sustainable Yield Estimator for Reservoirs in Massachusetts, 1960-2004 
Prepared by the Pembroke Publishing Service Center.

For more information concerning this report, contact:

Director

U.S. Geological Survey

Massachusetts-Rhode Island Water Science Center 10 Bearfoot Road

Northborough, MA 01532

dc_ma@usgs.gov

or visit our Web site at:

http://ma.water.usgs.gov 
